\title{
Zero for nine
}

Citation for published version (APA):

van der Wulp, N. Y. (2016). Zero for nine: reducing alcohol use during pregnancy via health counselling and Internet-based computer-tailored feedback. [Doctoral Thesis, Maastricht University]. Maastricht University. https://doi.org/10.26481/dis.20160303nw

Document status and date:

Published: 01/01/2016

DOI:

10.26481/dis.20160303nw

Document Version:

Publisher's PDF, also known as Version of record

\section{Please check the document version of this publication:}

- A submitted manuscript is the version of the article upon submission and before peer-review. There can be important differences between the submitted version and the official published version of record.

People interested in the research are advised to contact the author for the final version of the publication, or visit the DOI to the publisher's website.

- The final author version and the galley proof are versions of the publication after peer review.

- The final published version features the final layout of the paper including the volume, issue and page numbers.

Link to publication

\footnotetext{
General rights rights.

- You may freely distribute the URL identifying the publication in the public portal. please follow below link for the End User Agreement:

www.umlib.nl/taverne-license

Take down policy

If you believe that this document breaches copyright please contact us at:

repository@maastrichtuniversity.nl

providing details and we will investigate your claim.
}

Copyright and moral rights for the publications made accessible in the public portal are retained by the authors and/or other copyright owners and it is a condition of accessing publications that users recognise and abide by the legal requirements associated with these

- Users may download and print one copy of any publication from the public portal for the purpose of private study or research.

- You may not further distribute the material or use it for any profit-making activity or commercial gain

If the publication is distributed under the terms of Article $25 \mathrm{fa}$ of the Dutch Copyright Act, indicated by the "Taverne" license above, 


\section{Zero for nine:}

Reducing alcohol use during pregnancy

via health counselling and

Internet-based computer-tailored feedback

N.Y. van der Wulp 


\section{Colofon}

Cover design: de Merkbrouwerij

Lay-out: Ferdinand van Nispen, $m y$-thesis.nl

Production: GVO drukkers \& vormgevers B.V.

(C) N.Y. van der Wulp, 2016

ISBN: 978-90-6464-974-5

The research presented in this dissertation was conducted at the School for Public Health and Primary Care (CAPHRI), Department of Health Promotion, of Maastricht University. CAPHRI participates in CaRe, the Netherlands School of Primary Care Research. CAPHRI was classified as 'excellent' by the external evaluation committee of leading international experts that reviewed CAPHRI in December 2010.

This dissertation has been made possible thanks to the support of the Dutch Institute for Alcohol Policy STAP.

The research described in this dissertation was funded by the Netherlands Organization for Health Research and Development (ZonMW, Grant No. 120510005). 


\title{
Zero for nine:
}

\section{Reducing alcohol use during pregnancy via health counselling and Internet-based computer-tailored feedback}

\author{
PROEFSCHRIFT \\ ter verkrijging van de graad van doctor \\ aan de Universiteit Maastricht, \\ op gezag van de Rector Magnificus, \\ Prof. dr. L.L.G. Soete \\ volgens het besluit van het College van Decanen, \\ in het openbaar te verdedigen \\ op donderdag 3 maart 2016 om 14.00 uur
}

door

Nienke Yme van der Wulp 


\section{Promotor}

Prof. dr. H. de Vries

\section{Copromotor}

Dr. C. Hoving

\section{Beoordelingscommissie}

Prof.dr. N.K. de Vries (voorzitter)

Dr. C. Bolman (Open Universiteit Heerlen)

Prof.dr. R. Knibbe

Prof.dr. L. Lechner (Open Universiteit Heerlen)

Prof.dr. D. van de Mheen 


\section{Content}

$\begin{array}{lll}\text { CHAPTER } 1 \text { General Introduction } & 7\end{array}$

CHAPTER 2 Partner's influences and other correlates of 31 prenatal alcohol use

CHAPTER 3 Correlates of partner support to abstain from 51 prenatal alcohol use: A cross-sectional survey among Dutch partners of pregnant women

CHAPTER 4 A qualitative investigation of alcohol use 69 advice during pregnancy: Experiences of Dutch midwives, pregnant women and their partners

CHAPTER 5 Reducing alcohol use during pregnancy via health 97 counselling by midwives and internet-based computer-tailored feedback: A cluster randomized trial

CHAPTER 6 Dutch midwives' experiences with implementing 125 health counselling to prevent prenatal alcohol use

CHAPTER 7 General Discussion 133

Valorisation Addendum 159

References 169

Summary 184

Samenvatting 190

Dankwoord 197

Curriculum Vitae 202

Publication List 205 


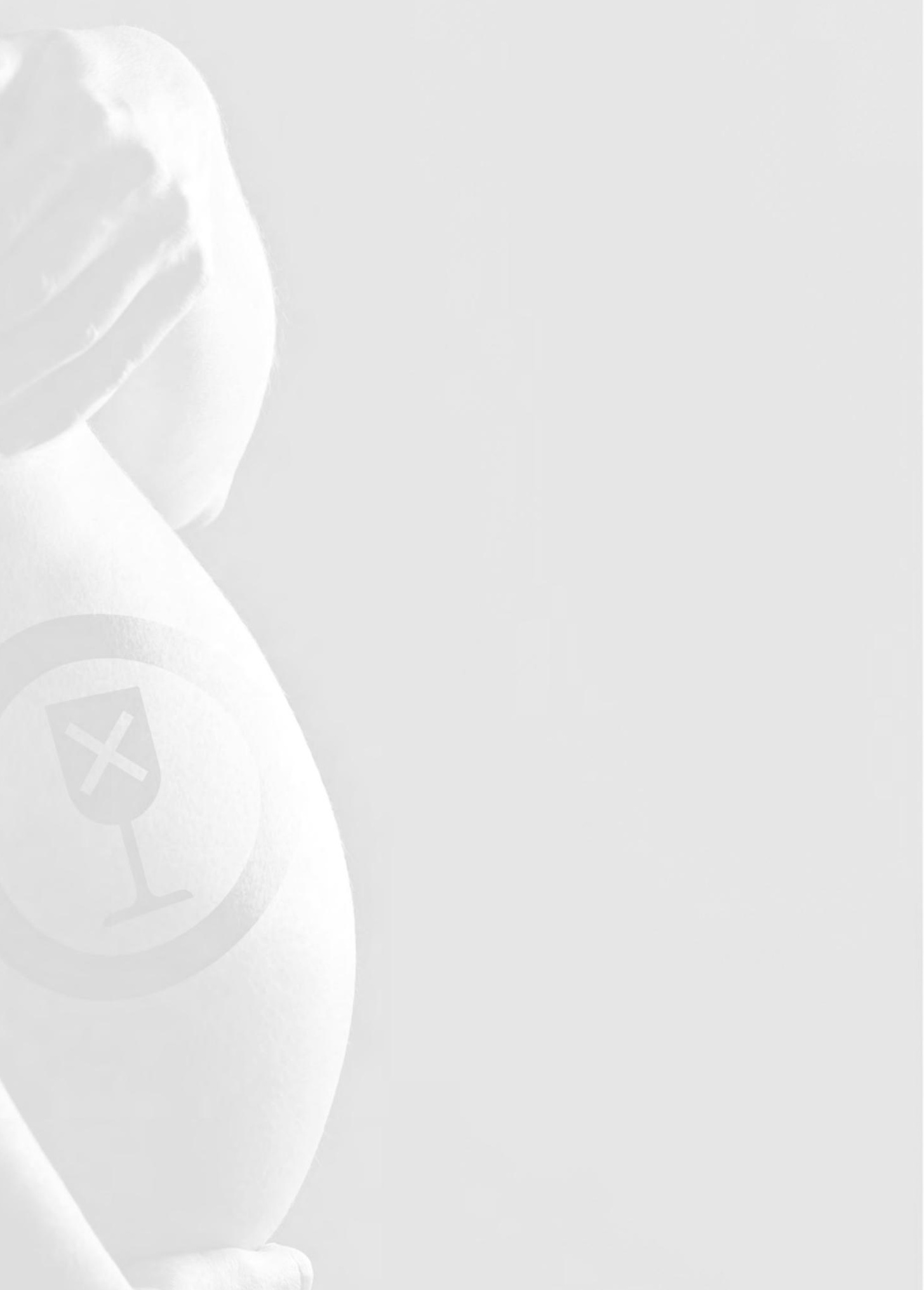




\section{CHAPTER 1}

General Introduction 



\section{Introduction}

"In my previous pregnancy I used to drink alcohol, and my child is perfectly healthy" is an often heard justification for pregnant women drinking alcohol. Many women drink alcohol in pregnancy, despite the risks. For example, because they believe their alcohol use is not risky for their child, or they know women who also used alcohol during their pregnancy and had not experienced any negative consequences (e.g. Jones, et al., 2011).

There is accumulating scientific evidence that even low levels of prenatal alcohol exposure can cause adverse neurobehavioral effects in children (Nathanson, Jayesinghe, \& Roycroft, 2007). This makes alcohol use in pregnancy one of the leading preventable causes of mental retardation in children (Centers for Disease Control and Prevention, 2002). Accordingly, in an advisory report published in 2005, the Health Council of the Netherlands concluded that no lower limit for alcohol use could be determined that would certainly have no effect on the foetus and the pregnancy (Health Council of the Netherlands, 2005). In the same report, the Health Council estimated that 35 to $50 \%$ of the Dutch pregnant women use alcohol.

The harmful effects of prenatal alcohol exposure in combination with the high number of Dutch, pregnant women using alcohol call for effective strategies to discourage prenatal alcohol use. In this dissertation two brief interventions to reduce prenatal alcohol use are described. This introductory chapter first elaborates on the need of an effective intervention to reduce prenatal alcohol use. Subsequently, more details are given of the pregnant women using alcohol and the alcohol advice they receive from health professionals. Then, the theoretical background, the strategies and the content of the interventions are described. Finally, the rationale for the studies on the development, effectiveness and implementation of the interventions presented in this dissertation is explicated. 


\section{The need for an effective intervention to reduce prenatal alcohol use}

The need for an effective intervention to reduce prenatal alcohol use is explained by reviewing the literature on the harmfulness of prenatal alcohol exposure and on how many pregnant women consume alcohol.

\section{The seriousness of the health problem: How risky is alcohol use in pregnancy?}

\section{The effects of alcohol on cellular processes during prenatal development}

After a woman consumes an alcoholic drink, the alcohol is absorbed in the gastrointestinal tract, where it enters the bloodstream. Alcohol is then distributed throughout the body via the circulatory system: as the blood circulates in the body, alcohol enters and dissolves in the liquid of each tissue in the body (Kerssemakers, et al., 2008). When a woman in her early pregnancy drinks alcohol, the alcohol dissolves in the embryonic body fluids. In a later stage of the pregnancy, alcohol can pass freely through the placenta, it enters the foetus' bloodstream and is then distributed throughout the body of the foetus via its circulatory system. The alcohol level of the foetus attains the same level as that of the mother and remains in the body until it is broken down by the liver of the mother (Idanpaan-Heikkila, et al., 1972), which takes approximately one to one-and-a-half hours for one standard glass of alcoholic beverage (van Faassen \& Niemela, 2011).

Alcohol in the foetus affects various cellular processes that occur during the foetal development. For example, alcohol can disturb the development of the cells of organs (proliferation and mitosis). Alcohol can alter the migration of cells leading to nerve cells arriving at abnormal locations. Alcohol can interfere with cell adhesion, which may have various consequences for the brains of the unborn baby, including mental retardation, complete absence of the corpus callosum (the white brain substance that connects the two hemispheres) or abnormal development of small brains. By altering the functions of certain neurotransmitter systems, alcohol can disorder the organization of the 
central nervous system during the foetal development. Finally, alcohol may contribute to excessive death of cells, for example in the brains (Goodlett \& Horn, 2001). Not all unborn babies will suffer in the same way from prenatal alcohol use. The likelihood and severity of these effects increase with the mother's quantity and frequency of alcohol intake, her number of pregnancies, age and unhealthy nutritional habits; they decrease with the mother's weight (May, et al., 2008), her genetics (e.g. presence of allele ADH1 B*3; Scott \& Taylor, 2007) and the speed with which her liver breaks down the alcohol (Chernoff, 1980). As not all risk and protective factors are known before birth, it cannot be calculated beforehand which consequences of prenatal alcohol use result from the interference of prenatal alcohol use with the cellular processes during prenatal development.

\section{Consequences of prenatal alcohol use during pregnancy and after birth} When prenatal alcohol use interferes with cellular processes of the developing foetus, several consequences can be expected, varying with the stage of the pregnancy in which alcohol is being consumed. During the first weeks of pregnancy, alcohol can increase the risk of miscarriage (Armstrong, McDonald, \& Sloan, 1992). In the first trimester, alcohol can deform body parts including the heart, arms, eyes, legs, teeth, palate, external genitals and ears, which are formed in this period. In the second and third trimester, alcohol consumption can have a negative influence on the growth of the foetus. As the central nervous system develops throughout the pregnancy, alcohol use during the whole pregnancy can cause neurological damage (Coles, 1994).

After birth, various effects of prenatal alcohol consumption may become visible. These effects are irreversible. Due to the negative influence of alcohol on the growth, the child may be smaller or lighter than average (e.g. Whitehead \& Lipscomb, 2003). Children exposed to prenatal alcohol may also have facial abnormalities. The most severely affected children have narrow eye slits, a smooth philtrum (gutter between nose and upper lip) and a thin upper lip. Other facial abnormalities may be a low nasal bridge, low ears, a narrow chin, abnormalities of the teeth and a high palate (e.g. Sulik, Cook, \& Webster, 1988). Finally, neurological damage can manifest itself in behavioural and neurological abnormalities 
(Chudley, et al., 2005). Common effects are poor social functioning, hyperactivity, mental retardation, learning disabilities, poor memory and autistic behaviour (Niccols, 2007).

When a child has been prenatally exposed to alcohol and has all three characteristics (i.e. smaller than average, facial abnormalities and neurological damage), it may be diagnosed with the chronic condition of Foetal Alcohol Syndrome (FAS; Bertrand, et al., 2004). If a child does not have all the features of FAS, there may be other diagnoses, such as Alcohol Related Neurodevelopmental Disorder (ARND; Bertrand, et al., 2004). The term Foetal Alcohol Spectrum Disorder (FASD) covers the whole area of defects caused by prenatal alcohol exposure (Streissguth \& O'Malley, 2000).

It is unclear how many people in the Netherlands suffer from an FASD, due to a lack of valid epidemiological studies. One study has investigated the prevalence of FASD by asking all Dutch paediatricians to report their experiences with FASD. This study, conducted in 2007 and 2008, revealed a total of 39 cases of FASD (van Wieringen, et al., 2009). The authors subsequently estimated that the prevalence of FASD in the Netherlands might be approximately 1 in every 10,000 births. However, self-report by paediatricians may be responsible for a large underestimation of actual cases, as not all children with an FASD may visit a paediatrician and not all paediatricians may be adequate in diagnosing FASD. A more accurate method is population-based, active case ascertainment in which FASD cases are sought through structured outreach in a defined population. In Europe, only four studies have been carried out using this method, involving two Italian studies (May, et al., 2006; May, et al., 2011) and two Croatian studies (Petkovic \& Barisic, 2010, 2013). In these studies, schoolchildren were screened for FAS and FASD. Depending on the criteria that were established, the Italian studies showed prevalence rates of FAS varying between 3.7 and 12.0 per 1,000 children and of FASD varying between 20.3 and 63.0 per 1,000 children; the Croatian studies showed FAS prevalence rates between 6.4 and 16.9 per 1,000 children and FASD prevalence rates varying between 34.0 and 66.7 per 1,000 children. Although the Dutch drinking pattern may differ from the 
drinking patterns in Italy and Croatia, of all research available, these studies provide the best applicable data to estimate the FASD prevalence in the Netherlands. Thus, until valid epidemiological studies have been conducted in the Netherlands, it can roughly be estimated that 2 to $6 \%$ of all Dutch children suffer from the consequences of prenatal alcohol use. With approximately 200,000 births each year (Statistics Netherlands, 2014), it can be estimated that 4,000 to 12,000 Dutch babies are born each year with an FASD.

\section{The magnitude of the health problem: How many pregnant women consume alcohol?}

The importance of conducting the present research does not only depend on the severity of the effects of prenatal alcohol use, but also on the number of women actually using alcohol in pregnancy. Unfortunately, there are no recent representative data available on prenatal alcohol use in the Netherlands, but merely on alcohol use of women of childbearing age, revealing that in $2012,80 \%$ of the women aged 16 to 50 years old consumed alcohol (Statistics Netherlands, 2013). In their advisory report on prenatal alcohol use, the Health Council of the Netherlands (2005) reported two older, non-representative Dutch studies examining the alcohol use of pregnant women. The largest study was conducted in 1978-1979 with 317 midwives gathering data from almost 3000 women on alcohol use during pregnancy, showing that $52 \%$ of the pregnant women used alcohol (De Jonge \& van der Klaauw, 1986; Verkerk, et al., 1993). The second study was conducted in 1987 with 142 pregnant women showing that $32 \%$ used alcohol during pregnancy (Tholen, 1987). Based on these two studies, the Health Council of the Netherlands estimated in 2005 that 35 to $50 \%$ of the Dutch pregnant women use alcohol (Health Council of the Netherlands, 2005).

Since the publication of the Health Council's report, four additional Dutch studies have published data on prenatal alcohol use, showing that the prevalence of prenatal alcohol use has not been subject to dramatic changes. These data collected between 2001 and 2009 provided 
prevalence rates which varied between $28.6 \%$ and $41.8 \%$ (Bakker, et al., 2010; Jentink, et al., 2011; Lanting, et al., 2009; Pfinder, et al., 2013). Although none of these studies are based on representative samples of Dutch pregnant women, these various studies jointly suggest that approximately $30 \%$ of the Dutch pregnant women use alcohol. This is much lower than the $71 \%$ prevalence in Denmark (Kesmodel, et al., 2003) and the 54\% prevalence in Ireland (Donnelly, et al., 2008), but much higher than the $14.2 \%$ prevalence in Germany (Pfinder, et al., 2013), the $8 \%$ prevalence in the United States (Centers for Disease Control and Prevention, 2012), and the 6\% prevalence in Sweden (Nilsen, et al., 2008). The approximate estimation of $30 \%$ of the Dutch pregnant women using alcohol stresses the need of developing an effective intervention to reduce prenatal alcohol use.

\section{Determinants of prenatal alcohol use}

When developing an intervention to reduce prenatal alcohol use, information is required on the target group: what are determinants of women who are drinking alcohol during pregnancy? In describing the current knowledge on these determinants, gaps in this knowledge are exposed, of which some are targeted in this dissertation.

Research has shown that pregnant women using alcohol differ in various aspects from pregnant women abstaining from alcohol (Skagerstrom, Chang, \& Nilsen, 2011). Most of the studies on determinants of prenatal alcohol use have focused on predisposing and awareness determinants, showing that women who continue to drink alcohol in pregnancy are older (e.g. Haynes, Dunnagan, \& Christopher, 2003; Nilsen, et al., 2008), have a higher education (e.g. Perham-Hester \& Gessner, 1997), drink more alcohol before pregnancy (e.g. Chang, et al., 2007), more often smoke cigarettes in pregnancy (e.g. Comasco, et al., 2012) and perceive lower risk of prenatal alcohol use for the unborn baby (Testa \& Reifman, 1996). A study conducted in Amsterdam further showed that pregnant women using alcohol are taller, have a more healthy BMI, less often have pregnancy hypertension, have lower mental distress and more often have a Dutch nationality (Pfinder, et al., 2013). 
In addition to predisposing and awareness determinants, several health behavioral models suggested that health behavior is also determined by motivational factors (e.g. Ajzen, 1985; De Vries, et al., 2003). Thus far, only a few studies have provided evidence on the relevance of motivational factors on prenatal alcohol use. Peadon and colleagues (2011) have shown that women with a neutral or positive attitude towards prenatal alcohol use are more likely to continue their alcohol use during pregnancy. In addition, women with a partner using alcohol more often consume alcohol when they are pregnant (Bakhireva, et al., 2011; Chang, et al., 2011; Waterson, Evans, \& Murray-Lyon, 1990). The scarcity of research on the role of motivational factors in reducing prenatal alcohol use is in contrast with the evidence that motivational factors play an important role in changing other health behavior (e.g. Ajzen, 1985; Bandura, 1986; Janz \& Becker, 1984; Schwarzer, Lippke, \& Luszczynska, 2011), such as for example smoking cessation (e.g. De Vries, et al., 1998) or attending cancer screening programmes (Knops-Dullens, et al., 2007). In order to compensate for this scarcity of attention, this dissertation focuses on these factors in general and social influences as one of these factors in specific.

The few studies on social influence and prenatal alcohol abstinence (Bakhireva, et al., 2011; Chang, et al., 2011; Waterson, Evans, \& MurrayLyon, 1990) providing first evidence of the importance of these factors in reducing prenatal alcohol use, paved the way for further research on social influence and prenatal alcohol use. Social influence incorporates three elements: social norm, social modelling and social pressure (De Vries, et al., 1995). Social norm refers to the perceived opinions of important others around the respondent, such as her partner. Social modelling refers to perceived behaviour of important others. Social pressure is regarded as a respondent's perceived pressure from important others to behave in a certain way (De Vries, et al., 1995). The pregnant woman's partner can be regarded as an important other affecting the pregnant woman's behaviour (Ockene, et al., 2002). Thus far, current research has merely provided evidence that modelling of the partner has an effect on prenatal alcohol use (Bakhireva, et al., 2011; Chang, et al., 2011; Waterson, et al., 1990). Firm scientific evidence for the effect of partner's norm and support on prenatal alcohol use is still lacking. 
In most studies on partner's behavior and prenatal alcohol use, social influence relates to the respondent's perception of this influence. It is unclear whether this truly reflects partner's actual behavior. Only one study investigated the relation between the pregnant woman's perception and her partner's report of his support (McNamara, et al., 2006), showing merely a slight agreement in support scores. In addition, there is no evidence that perceived partner's behaviour is a stronger determinant of prenatal alcohol use than actual behaviour.

Finally, when research shows that partners can play an important role in interventions targeting the prevention of prenatal alcohol use, a better understanding of the determinants of partner support to abstain from prenatal alcohol use can help to make a better use of partner support. Thus far, no previous study has looked into the determinants of partner support to abstain from prenatal alcohol use.

In order to fill this gap of missing information on motivational factors in general and the role of the partner in specific, the present dissertation aimed to answer the following research questions:

1. What are motivational determinants of prenatal alcohol use?

2. How do partner's norm, modelling and support as perceived by the pregnant woman and reported by her partner relate to prenatal alcohol use?

3. How are perceived and reported partner's behaviour regarding prenatal alcohol use related to each other?

4. How important are perceived and reported partner's behaviour in explaining alcohol use of pregnant women?

5. What are determinants of partner support to abstain from prenatal alcohol use? 


\section{Advice on alcohol use during pregnancy}

In order to develop an intervention with information helping pregnant women to reduce their prenatal alcohol use, it is important to know about the current situation with regard to alcohol advice. Consequently important questions are what alcohol advice do pregnant women currently receive, from who do they receive this advice and how is this advice experienced, both by the provider and the receiver of this advice? Reviewing the literature on alcohol advice during pregnancy clarifies the gaps in knowledge on this issue, some of which are targeted in this dissertation.

Research has shown that the absence of alcohol abstinence advice from health professionals presents an environmental risk factor of prenatal alcohol use (Jones, et al., 2011; Kesmodel \& Schioler Kesmodel, 2002; Raymond, et al., 2009). Both pregnant women and health professionals have reported flaws in health professionals' alcohol advice. International studies revealed that many pregnant women are dissatisfied with their health professionals' alcohol advice (Jones, et al., 2011; Kesmodel \& Schioler Kesmodel, 2002; Raymond, et al., 2009), they often do not recall being actively engaged by the midwife in a discussion about alcohol consumption (Jones, et al., 2011) and when they receive alcohol advice, they describe this advice as being confusing, unclear or lacking sufficient detail (Jones, et al., 2011; Kesmodel \& Schioler Kesmodel, 2002; Raymond, et al., 2009). Pregnant women's negative experiences with health professionals' alcohol advice are supported by research showing the difficulties of midwives with providing alcohol advice (Kesmodel \& Kesmodel, 2011). Midwives find it difficult to screen for prenatal alcohol use (Holmqvist \& Nilsen, 2010), they believe they lack detailed knowledge about the risks of alcohol use in pregnancy and lack skills to deliver advice without distressing women who have already used alcohol in pregnancy (Kesmodel \& Kesmodel, 2011).

Although, international studies have shown that midwives experience difficulties with providing alcohol advice (Holmqvist \& Nilsen, 2010; Jones, et al., 2011; Kesmodel \& Kesmodel, 2011), there is no research 
available on Dutch midwives' alcohol advice. In the Netherlands, the midwife can be considered an important health professional for pregnant women as she is the first responsible health professional for the pregnant woman as long as there are no complications (Wiegers, Van der Velden, \& Hingstman, 2005). Midwives provide care to $75 \%$ of all Dutch pregnant women at the beginning of their pregnancy (Wiegers, et al., 2005). According to the guidelines of the Royal Dutch Organization of Midwives (KNOV), midwives should recommend their clients to abstain from alcohol during pregnancy (de Boer \& Zeeman, 2008). However, due to the lack of Dutch research, it is presently unclear whether Dutch midwives actually recommend alcohol abstinence. In addition, due to the differences between midwifery systems (McKay, 2004; Kateman \& Herschderfer, 2005), it is unclear whether the difficulties experienced by midwives revealed in international studies are also apparent in the Dutch situation.

International research has also shown that pregnant women are dissatisfied with midwives' alcohol advice (Jones, et al., 2011; Kesmodel \& Schioler Kesmodel, 2002; Raymond, et al., 2009). Again, it is unclear whether this dissatisfaction is also present among Dutch pregnant women.

Finally, as partners of pregnant women are considered an important social influence in the prevention of prenatal alcohol use, it is important to know how they evaluate midwives' alcohol advice. Unfortunately, no study, in the Netherlands or abroad, has thus far investigated whether partners are included in midwives' alcohol advice and how they respond to such advice.

As more knowledge is needed on the current state of Dutch midwives' alcohol advice, the present dissertation aimed to answer the following research questions:

6. What alcohol advice do Dutch midwives give?

7. Which difficulties do Dutch midwives experience with providing alcohol abstinence advice? 
8. How do Dutch pregnant women experience midwives' alcohol advice?

9. How do partners of pregnant women experience midwives' alcohol advice?

\section{The theoretical background of the interventions to reduce prenatal alcohol use}

The seriousness and magnitude of the health problem justify the development of an intervention to reduce prenatal alcohol use. In this dissertation two brief interventions to reduce prenatal alcohol use are described. For these interventions to be effective, they should focus on the most important and changeable determinants of the health behaviour under consideration. The I-Change Model was used as framework to identify these determinants (Figure 1.1 and Chapter 4).

The I-Change model has been applied within several previous Dutch studies, for example concerning smoking in pregnancy (De Vries, et al., 2006a), sunscreen use (De Vries, et al., 2012), cancer screening (Knops-Dullens, de Vries, \& de Vries, 2007) and breast-feeding (Kools, et al., 2005b). It is an integration of various behaviour-oriented theories such as the Social Cognitive Theory (Bandura, 1986), the Health Belief Model (Janz \& Becker, 1984), the Theory of Planned Behaviour (Ajzen, 1985), the Transtheoretical Model (Prochaska \& Velicer, 1997), and Goal Setting theories (Gollwitzer, 1999; Schwarzer, Lippke, \& Luszczynska, 2011). The model maps the psychological determinants of behaviour change, distinguishing three motivational change phases (De Vries, et al., 2003). In the pre-motivational phase, people need to become aware of a health problem. In this phase, preceding or predisposing factors (e.g. demographics, prenatal alcohol use and pregnancy characteristics), information factors (e.g. messages and sources) and awareness factors (e.g. knowledge and risk perceptions) are important determinants. For instance, people may become aware of the risks of prenatal alcohol use when they have more knowledge about the consequences of prenatal alcohol use for the unborn baby. In the motivational phase, people need 
to become motivated to change their behaviour. In this phase, attitudes, social influence perceptions and self-efficacy expectations are important. For instance, people who perceive social support from important others may be more motivated to change their prenatal alcohol use. Finally, in the post-motivational phase, people need to translate intentions into actions. For example, pregnant women are more successful to reduce their prenatal alcohol use when they have formulated preparatory action plans. In order to achieve behaviour change, all of these premotivational, motivational and post-motivational determinants differentiated by the I-Change Model are addressed in this dissertation.

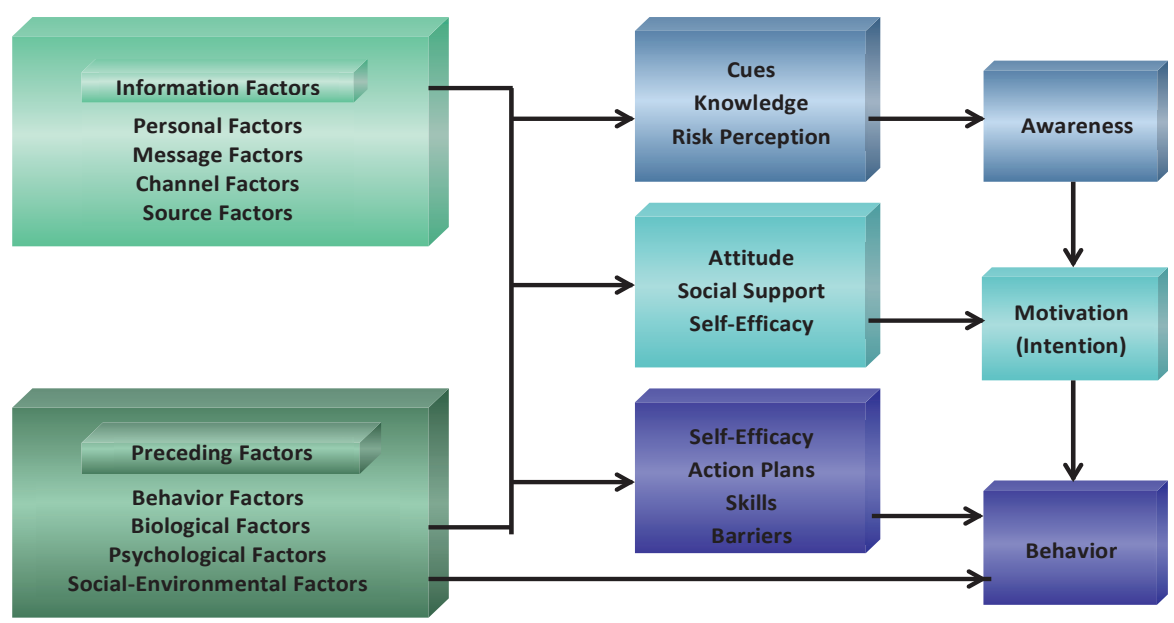

Figure 1.1 The I-Change Model 2.1

\section{The strategies of the interventions to reduce prenatal alcohol use}

The two interventions described in this dissertation differed from each other in what kind of strategy was applied to change the targeted health behaviour.

Health Counselling $(\mathrm{HC})$ is a strategy that has often been used for brief alcohol interventions. HC is a therapy provided by a health professional 
to an individual or group to prevent or treat health issues, including substance abuse and addiction (Joffe \& Radius, 1991). In international studies (Chang, et al., 1999; Handmaker, Miller, \& Manicke, 1999; O'Connor \& Whaley, 2007; Reynolds, et al., 1995), HC has been effective in reducing prenatal alcohol consumption, as has been shown by a recent review on psychological and educational interventions to reduce alcohol consumption by pregnant women (Stade, et al., 2009). Although HC has successfully been used in a prenatal smoking cessation intervention provided by Dutch midwives (De Vries, et al., 2006a), currently, there is no effective HC intervention available for Dutch midwives who want to help their clients to change their alcohol use. A drawback of HC is the large responsibility of health professionals to adequately implement the intervention. Previous research on smoking cessation in pregnancy (Lumley, et al., 2009) has shown that health professionals' lack of time (Hajek, et al., 2001), pessimism about the effectiveness of the intervention (McLellan, et al., 2000), perceived lack of skills and training (De Vries, et al., 2006a) and administrative barriers (Strand, et al., 2003) were disadvantageous for the efficacy of the intervention.

This drawback of health professionals' responsibility for an adequate implementation can be overcome by an alternative and promising strategy, called computer tailoring (CT; De Vries \& Brug, 1999). As with $\mathrm{HC}$, the content of this advice is based on the answers of respondents to questions relating to a certain behaviour. In contrast to $\mathrm{HC}$, the advice is not delivered face-to-face by a health professional, but generated by a computer program. As the feedback is adapted to the specific characteristics of a particular individual, it yields the potential to provide messages highly tailored to the individuals' situation (Hawkins, et al., 2008). CT messages have been shown to attract and keep an individual's attention (De Vries \& Brug, 1999; Kreuter, 1999) more than generic advice, resulting in a more thorough processing of information (Dijkstra, 2005). CT has proven to be effective in changing different health related behaviours (De Vries, et al., 2008; Krebs, Prochaska, \& Rossi, 2010), like smoking (E.S. Smit, de Vries, \& Hoving, 2012), vegetable and fruit intake (Oenema, Tan, \& Brug, 2005) and alcohol use (Boon, et al., 2011; Schulz, et al., 2013). To our knowledge, only one CT intervention for alcohol use 
in pregnancy was developed (Tzilos, Sokol, \& Ondersma, 2011). Tzilos and colleagues found that users liked the program and appreciated the ease of use. Nevertheless, they did not find any significant difference on the reduction of alcohol use compared to an assessment-only condition.

Summarizing, HC has already shown to be effective in preventing prenatal alcohol use, but there is no effective HC protocol for Dutch midwives. As HC is demanding for health professionals, CT is an interesting alternative. However, the effectiveness of $\mathrm{CT}$ has thus far only been shown in other kinds of health behaviour. Based on these considerations, it was decided to develop an HC and a CT intervention to reduce alcohol consumption during pregnancy.

\section{The content of the interventions to reduce prenatal alcohol use}

A brief description of the interventions to reduce prenatal alcohol use which have been developed in this dissertation is provided in the following paragraphs.

\section{$H C$ intervention}

The HC protocol was based on a health counselling protocol which has previously shown to be effective in smoking cessation during pregnancy (De Vries, et al., 2006a).

The HC protocol consisted of seven steps to be addressed by midwives in three feedback sessions. Feedback session 1 took place in the initial consultation of the midwife with the pregnant woman using alcohol and consisted of the first five steps. In step 1, the midwife assessed the amount and frequency of alcohol use of the pregnant woman before and during pregnancy and of her partner during pregnancy. She discussed with the pregnant woman whether she was motivated to stop drinking alcohol during her pregnancy. In step 2, the midwife asked women 
who were strongly motivated to stop drinking alcohol to state the advantages of quitting. She discussed the disadvantages of alcohol use and the advantages of stopping with women who were moderately or not motivated to stop drinking alcohol. During this step, the midwife advised the pregnant woman to stop drinking alcohol. In step 3, the midwife discussed the barriers for successful quitting. In addition, she discussed how a significant other could help the participant when she found it difficult to abstain from alcohol. In step 4, a self-help guide was provided, which was adapted from an intervention on smoking in pregnancy (De Vries, et al., 2006a). Additionally, the midwife mentioned relevant websites where the pregnant woman could find additional information on the consequences of prenatal alcohol use and tips on how to stop drinking. The midwife informed the participant how she could develop action plans for quitting and coping plans with problems she might encounter when trying not to drink alcohol. In step 5, the midwife asked participants to set a date for quitting alcohol consumption.

Feedback session 2 took place approximately six weeks after feedback session 1 and consisted of step 6 . In this step, the midwife reassessed the alcohol use of the pregnant woman. If the pregnant woman had stopped drinking alcohol, the midwife talked with her about her experiences with alcohol abstinence; if the pregnant woman had not stopped drinking alcohol, the midwife explained her that she was still capable of stopping. The midwife and the pregnant woman discussed difficult situations and coping plans.

Feedback session 3 took place approximately six weeks after feedback session 2 and consisted of step 7. In this step, the midwife again assessed the participant's alcohol use. She discussed with the participant why it was important to remain abstinent from alcohol during the remainder of her pregnancy and provided tips for maintaining this behaviour change. In addition, she discussed the implications of alcohol use for breastfeeding. 


\section{CT intervention}

The CT intervention was developed using Tailorbuilder software (OSE, the Netherlands), a program which is specifically designed to develop web-based computer-tailored interventions.

Similar to the HC intervention, the CT intervention consisted of three feedback sessions. Feedback 1 was given after the baseline questionnaire following registration. This feedback was tailored to the determinants of the participant assessed in the baseline questionnaire. First, after the assessment of the participant's alcohol use, factual information was provided on the consequences of alcohol use during pregnancy. Then, by providing feedback on participant's responses to the absolute and comparative measures of risk perception, risk perception was used to increase participant's motivation to stop drinking alcohol. Participant's attitude on alcohol abstinence during pregnancy was used to confirm the perceived advantages and counter the perceived disadvantages. Social influences were reflected on and participants were encouraged to consider what kind of social support was needed. Subsequently, situations for which the participants had the lowest level of confidence that they would abstain from alcohol were assessed and reflected on. Then, participants were stimulated to make action and coping plans to carry out the behaviour change and cope with difficult situations. Finally, participants set a date for quitting their alcohol use.

Feedback 2 took place six weeks after baseline and was once more based on the assessment of participant's determinants in the baseline questionnaire. Specifically, the participant could choose one or more characteristics assessed with the baseline questionnaire (for example, risk perception or attitude) and compared to Feedback 1, received more elaborate information on her selection of characteristics. For example with regard to attitude, Feedback 1 consisted of general information on the advantages of alcohol abstinence and disadvantages of prenatal alcohol use. Accordingly, it was stated that a positive attitude towards alcohol abstinence would help the participant to abstain from alcohol. In contrast, in Feedback 2, participants received specific item-based 
feedback. For example, participants who considered alcohol abstinence advantageous for the development of the baby were confirmed in their answer and were informed that prenatal alcohol use may impede the baby's development by causing physical or mental impairment.

Feedback 3 took place six weeks after Feedback 2. In this session, the participant's alcohol use and her determinants to abstain from prenatal alcohol use were reassessed. The participant received feedback which was tailored to changes with the determinants assessed in the baseline questionnaire. Information was presented on why it is important to remain abstinent from alcohol during the remainder of her pregnancy and tips were provided for maintaining this behaviour change. Participants were again given the opportunity to make action and coping plans to carry out the behaviour change and cope with difficult situations.

\section{Pre-tests of the interventions}

The midwives' protocol of the HC intervention was pretested among five midwives and the $\mathrm{CT}$ intervention was pretested among five pregnant women using alcohol. The pre-tests yielded useful findings, for example about unclear questions and formulations both in the protocol and in the $\mathrm{CT}$ intervention. In addition, the pre-test of the $\mathrm{CT}$ intervention revealed that pregnant women using alcohol are highly sensitive when they believe that their alcohol use is being overestimated or when they sense that they are thought to be alcohol dependent. These findings led to an improvement of the texts in the final versions of the HC protocol and $\mathrm{CT}$ intervention.

\section{Effect evaluation}

After the development of the two interventions to reduce prenatal alcohol use, a plan for program use was developed, including adoption and implementation. In this plan, the collaboration with midwives and their professional association (KNOV) was central. Several large-scale 
mailings, supported by the KNOV, promotion in the Dutch Journal for Midwifery, expert meetings and conferences informed midwives about the interventions. Midwifery practices interested in the interventions could sign up for participation and refer their clients to the intervention website. On this website, pregnant women could register and were subsequently assigned to one of the interventions. The midwives of pregnant women assigned to the HC intervention were notified of their clients' participation, implying that they could start the HC intervention with these clients; the pregnant women assigned to the CT intervention automatically received programmed emails with CT feedback.

The effectiveness of the $\mathrm{HC}$ and $\mathrm{CT}$ intervention was tested with a threearm cluster randomized trial comparing a $\mathrm{HC}$ and a CT group to a control group, receiving usual care (UC) from their midwives. Measurements took place at baseline (T0) and at three (T1) and six months (T2) after baseline. During all measurements, participants had to complete an online questionnaire via the study website. The primary outcome of the study was alcohol use during pregnancy (yes/no) at three and six month follow-up. The secondary outcome was the average weekly alcohol consumption since the previous questionnaire, assessed with the Quantity Frequency-Variability (QFV) questionnaire (Lemmens, Tan, \& Knibbe, 1992), also at three and six month follow-up.

With this effect evaluation, the present dissertation aimed to answer the following research questions:

10. Is HC provided by midwives effective for Dutch women to stop or reduce their alcohol use in pregnancy?

11. Is CT effective for Dutch women to stop or reduce their alcohol use in pregnancy?

In this project, also a process evaluation was conducted to assess the extent of the implementation of the $\mathrm{HC}$ intervention by midwives. For this purpose, semi-structured telephone interviews were conducted with all midwives who participated in the effectiveness trial. This evaluation took place after the 6 month follow-up measurement of the 
HC participants. Experiences of midwives with the implementation of a brief intervention to reduce prenatal alcohol use provide valuable information for developers of future brief interventions (e.g. Wilson, et al., 2012). Although research in the related field of smoking cessation during pregnancy has shown that difficulties for health professionals can be detrimental for the implementation of a $\mathrm{HC}$ intervention (Lumley, et al., 2009), there is a lack of data on process evaluations of $\mathrm{HC}$ interventions to prevent prenatal alcohol use (Stade, et al., 2009). It is also important to assess the implementation of the $\mathrm{CT}$ intervention. However, within the time available to conduct this research, this was unfortunately impossible.

With regard to the process evaluation, the final research question addressed by this dissertation is:

12. What are midwives' experiences with the implementation of a brief health counselling intervention to prevent prenatal alcohol use?

\section{Outline of this dissertation}

In this dissertation the development, evaluation and implementation of two brief interventions to prevent prenatal alcohol use are described.

Chapter 2 presents an online cross-sectional study among 158 Dutch pregnant women and their partners. This quantitative study aimed to investigate the influence of partners on pregnant women's alcohol consumption within the context of other factors. This chapter answers research questions 1 to 4 .

1. What are motivational determinants of prenatal alcohol use?

2. How do partner's norm, modelling and support as perceived by the pregnant woman and reported by her partner relate to prenatal alcohol use? 
3. How are perceived and reported partner's behaviour regarding prenatal alcohol use related to each other?

4. How important are perceived and reported partner's behaviour in explaining alcohol use of pregnant women?

Chapter 3 presents an online cross-sectional study among 237 Dutch partners of pregnant women. This quantitative study aimed to identify determinants of this partner support to abstain from prenatal alcohol use by analysing differences between partners reporting low versus high support. This chapter answers research question 5.

5. What are determinants of partner support to abstain from prenatal alcohol use?

Chapter 4 answers research questions 6 to 9 by presenting two qualitative studies. The first study among 10 midwives aimed to explore what alcohol advice Dutch midwives give to their clients. The second study among 25 pregnant women and 9 partners aimed to explore what information Dutch pregnant women and partners receive about alcohol in pregnancy.

6. What alcohol advice do Dutch midwives give?

7. Which difficulties do midwives experience with providing alcohol abstinence advice?

8. How do pregnant women experience midwives' alcohol advice?

9. How do partners of pregnant women experience midwives' alcohol advice?

Chapter 5 presents the effect evaluation of two brief interventions to prevent prenatal alcohol use. In this cluster randomized trial, sixty Dutch midwifery practices recruited 135 health counselling (HC), 116 computer tailoring (CT) and 142 Usual Care (UC) respondents. This chapter answers research questions 10 and 11.

10. Is HC provided by midwives effective for Dutch women to stop or reduce their alcohol use in pregnancy? 
11. Is CT effective for Dutch women to stop or reduce their alcohol use in pregnancy?

Chapter 6 presents the process evaluation of the HC intervention. This qualitative study among 14 midwives aimed to explore midwives' experiences with the implementation of the $\mathrm{HC}$ intervention to prevent prenatal alcohol use. This chapter answers the final research question 12.

12. What are midwives' experiences with the implementation of a brief health counselling intervention to prevent prenatal alcohol use?

Chapter 7, the final chapter of this dissertation, discusses the results of the studies in this dissertation. Important findings are presented in relation to other relevant studies. Methodological limitations of the studies in this dissertation are also discussed. Based on the findings from the research questions, five concrete recommendations are formulated that appear promising to reduce prenatal alcohol use. 


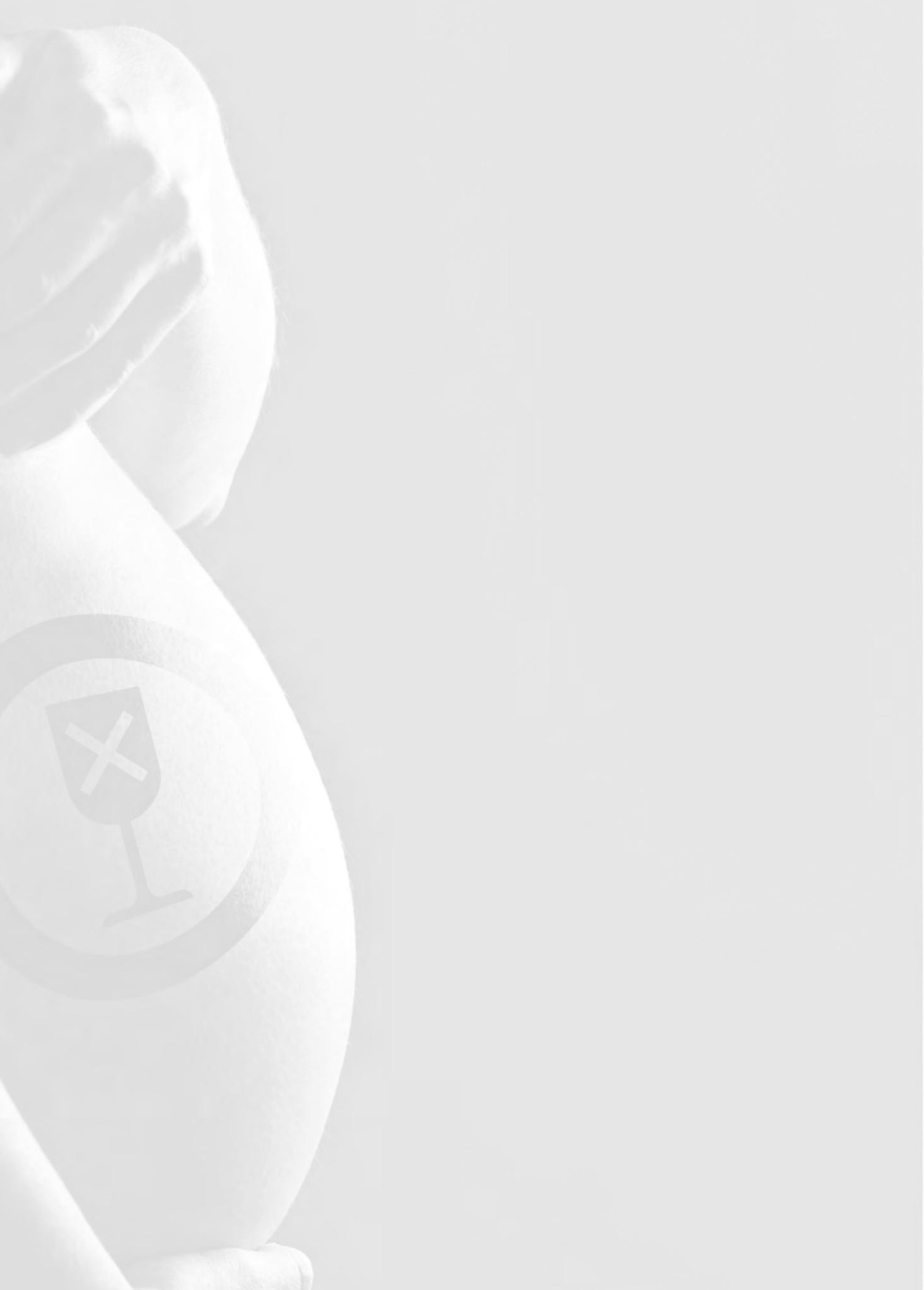




\section{CHAPTER 2}

\section{Partner's influences and other correlates of prenatal alcohol use}

Published as: Van der Wulp, N.Y., Hoving, C., de Vries, H. (2015).

Partner's influences and other correlates of prenatal alcohol use.

Maternal and Child Health Journal, 19(4): 908-916. 


\section{Abstract}

Objectives: To investigate the influence of partners on alcohol consumption in pregnant women within the context of other factors.

Methods: A Dutch nationwide online cross-sectional study among 158 pregnant women and their partners was conducted. To identify correlates of prenatal alcohol use, including perceived and reported partner norm (i.e. partner's belief regarding acceptability of prenatal alcohol use), partner modeling (i.e. partner's alcohol use during the woman's pregnancy) and partner support (i.e. partner's help in abstaining from alcohol during pregnancy), independent sample T-tests and chi-square tests were conducted. Correlation analyses tested the relationship between perceived and reported partner influence. Multivariate logistic hierarchical regression analyses tested the independent impact of partner's perceived and reported influence next to other correlates from the I-Change Model.

Results: Pregnant women who consumed alcohol perceived a weaker partner norm $(\mathrm{p}<.001)$ and less partner modeling $(\mathrm{p}<.05)$, with the partner reporting a weaker norm $(\mathrm{p}<.001)$, more drinking days per week $(\mathrm{p}<.05)$ and weaker support $(\mathrm{p}<.05)$. Perceived and reported partner norm, modeling and support were positively related (respectively $\mathrm{p}<$ $.01, \mathrm{p}<.01$ and $\mathrm{p}<.05$ ). The multivariate analyses demonstrated that pregnant women with a higher education who perceived lower severity of harm due to prenatal alcohol use and a weaker partner norm were more likely to use alcohol $\left(\mathrm{R}^{2}=.42\right)$.

Conclusions: This study demonstrated that perceived partner norm was the most critical of the constructs of perceived and reported partner influences in explaining prenatal alcohol use. 


\section{Introduction}

Alcohol use by pregnant women is one of the leading preventable causes of damage to an unborn child (Centers for Disease Control and Prevention, 2002). Because no safe alcohol intake level during pregnancy has been established (Streissguth \& O'Malley, 2000), abstaining from alcohol during pregnancy is highly recommended in many countries (O'Leary, et al., 2007). Still, many pregnant women continue to drink alcohol, with estimates ranging from 35 to 50 percent in the Netherlands (Health Council of the Netherlands, 2005). Effective interventions must be developed to reduce prenatal alcohol use and the identification of critical correlates of prenatal alcohol use may aid in the development of such interventions.

Various studies have previously investigated correlates of prenatal alcohol use (Skagerstrom, Chang, \& Nilsen, 2011). Women who continue to drink alcohol during pregnancy are older (e.g. Haynes, Dunnagan, \& Christopher, 2003; Nilsen, et al., 2008), have a higher education (e.g. Perham-Hester \& Gessner, 1997), drink more alcohol before pregnancy (e.g. Chang, et al., 2007), and smoke cigarettes more often during pregnancy (e.g. Comasco, et al., 2012) compared to women who do not use alcohol during pregnancy. Lower perceived risk of prenatal alcohol use (Testa \& Reifman, 1996) and a neutral or positive attitude toward prenatal alcohol use (Peadon, et al., 2011) appeared to predict heavier drinking. All of the previous studies concentrated on maternal characteristics. However, in line with theoretical models that emphasize the importance of social influence in determining health behavior (Ajzen, 1985; De Vries, et al., 1995), a growing body of research has additionally focused on the role of the partner in prenatal alcohol use.

Research on the effects of partners on prenatal alcohol use in women can be classified according to three constructs of social influence: partner norm (i.e. the partner's belief regarding acceptability of prenatal alcohol use), partner modeling (i.e. the partner's own alcohol use during the woman's pregnancy) and partner support (i.e. the partner's help in abstaining from alcohol during pregnancy; De Vries, et al., 1995). 
One study which examined partner norm demonstrated that partners consented to prenatal alcohol use more often than pregnant women (van der Wulp, Hoving, \& de Vries, 2013). Research focusing on partner modeling demonstrated that a partner's alcohol use is positively associated with prenatal alcohol use (Bakhireva, et al., 2011; Chang, et al., 2011; Waterson, Evans, \& Murray-Lyon, 1990). Finally, with regard to partner support, Chang and colleagues (2005) demonstrated that a brief intervention to reduce prenatal alcohol use is more effective when a partner chosen by the pregnant woman participated in the intervention and explicitly stated how he or she could support the pregnant woman in abstaining from prenatal alcohol use.

Although previous studies have yielded important information about the correlates of prenatal alcohol use, questions regarding a partner's influence on prenatal alcohol use in women remain. Thus far, no study has simultaneously investigated partner norm, modeling and support, in addition to the previously reported maternal characteristics. Moreover, most studies rely on the perception of the pregnant woman in assessing her partner's influence, although the woman's perception may differ from her partner's view. Finally, this study seeks to determine the relative impact of a partner's influence as perceived by the pregnant woman versus as reported by the partner in order to understand whether perceived and reported partner influence should be included in interventions targeting prenatal alcohol use.

By identifying important correlates of prenatal alcohol use, this study strives to assist in the development of effective interventions to reduce prenatal alcohol use and improve children's health. This study used the Integrated Model for Motivational and Behavioral Change (I-Change Model; De Vries, et al., 2003) as the theoretical framework. This model posits that a person's behavior is the result of intentions and abilities as determined by motivational factors such as attitudes, social influences (De Vries, et al., 1994) and self-efficacy (Bandura, et al., 1988; De Vries, Dijkstra, \& Kuhlman, 1988). The motivational factors are in turn determined by various predisposing factors (e.g. demographics and pregnancy characteristics), information factors (e.g. messages and sources) and awareness factors (e.g. knowledge and risk perceptions). 


\section{Methods}

\section{Procedure}

Respondents of this cross-sectional study were pregnant women and their partners, recruited through Dutch midwife practices in September and October of 2009. In the Netherlands, the midwifery system plays a pivotal role among Dutch pregnant women. Approximately 75 percent of Dutch women at the beginning of their pregnancy and 36 percent of Dutch women receive care exclusively from a midwife (Wiegers, van der Velden, \& Hingstman, 2005). All midwife practices in the Netherlands (approximately 500) were asked to distribute flyers among their clients and respective partners. Each flyer contained separate informational sections for the client and her partner, plus two separate login-codes for each to gain access to the online study questionnaire.

After three weeks, a reminder e-mail was sent to all of the midwife practices. The questionnaires were completed anonymously; however, the combination of log-in codes allowed for pairing of the couples. The number of couples invited to participate by each midwife practice is uncertain. Moreover, because the names of the participating midwife practices were not identified in the online questionnaire, the number of couples per practice who took part in the study remains unclear. Ethics approval for this study was granted by a human research ethics committee (METC Atrium MC - Maaslandziekenhuis; 08-N-50).

\section{Questionnaires}

Questionnaires for both the pregnant woman and her partner - which are available upon request - were based on questionnaires from previous studies that applied the I-Change Model (De Vries, et al., 2006a; Dijkstra, de Vries, \& Bakker, 1996; Dijkstra, De Vries, \& Roijackers, 1998a; Hoving, Mudde, \& de Vries, 2007a; Hoving, et al., 2010). 


\section{Questionnaire for Pregnant Women}

Pregnant women's predisposing factors were measured by four items assessing demographics: age, education ( 1 = Low: primary school/basic vocational school; 2 = Medium: secondary vocational school/high school degree; 3 = High: higher vocational school/college degree/university degree), income ( $1=€ 850$ or less; $2=€ 850$ to $€ 3500 ; 3=$ more than $€ 3500$ ), and marital status. Three items assessed pregnancy characteristics: first pregnancy $(0=$ no; $1=$ yes $)$, number of weeks pregnant, and complications in previous pregnancy ( $0=$ no/not applicable; $1=$ yes $)$. Four items assessed alcohol use and cigarette smoking before and during pregnancy $(0=$ no; $1=$ yes $)$.

Information factors were measured by one item, which acquired as to whether the midwife had or had not given alcohol abstinence advice (What advice regarding alcohol consumption did you receive from your midwife? 0 = no advice/advice to drink only small amounts of alcohol/ different advice/I don't know; 1 = advice to abstain from alcohol).

Awareness factors were measured by questions relating to knowledge and risk perception. Knowledge was measured by nine statements ( 0 = false/don't know; $1=$ true) relating to the harmfulness of alcohol consumption during pregnancy (e.g. Alcohol use during pregnancy can lead to brain damage of the unborn child). A total knowledge score was calculated by adding the number of items that were correctly answered. Risk perception was measured by two items: perceived likelihood that the baby would experience harm ( 1 = very low; 5 = very high); and perceived severity of harm to the baby ( $1=$ not serious; 5 = very serious) resulting from prenatal consumption of one standard glass of alcohol (that is, 10 grams of alcohol) per month.

Motivational factors were measured by questions pertaining to attitude, social influences and self-efficacy. Participants' attitude toward not drinking alcohol during pregnancy was assessed using 13 items, which were measured on a 5 -point Likert scale ( $1=$ fully disagree; $5=$ fully agree). Six questions measured the advantages of not drinking alcohol during pregnancy (e.g. Not drinking alcohol in pregnancy is better for 
my baby's health; $\alpha=.81$ ), and seven questions measured disadvantages (e.g. If I do not drink alcohol in pregnancy I feel more stressed; $\alpha=.83$ ). Social influences were assessed using three items, which were measured on a 5 -point scale ( $1=$ fully disagree; $5=$ fully agree) to determine the pregnant woman's perception of her partner's influence. These items concerned perceived norms (My partner considers it important that I do not drink alcohol in pregnancy), perceived modeling (My partner does not drink alcohol in my presence) and perceived support (My partner supports me in drinking no alcohol during pregnancy). Self-efficacy was measured by ten items asking respondents to indicate the level of difficulty with abstaining from alcohol during pregnancy on a 5-point scale ( 1 = very difficult; 5 = not difficult at all). The scree plot of a factor analysis using oblimin rotation revealed two underlying factors that explained 67 percent of the variation. Five items loaded on the social self-efficacy factor (e.g. When my friends are drinking alcohol; $\alpha=.87$ ); and five items loaded on the stress self-efficacy factor (e.g. When I am feeling sad; $\alpha=.85$ ).

Prenatal alcohol use by the pregnant woman was the outcome variable. Because even light alcohol use may pose a risk to the fetus (Nathanson, Jayesinghe, \& Roycroft, 2007), and complete alcohol abstinence is recommended in many countries (O'Leary, et al., 2007), including the Netherlands (Health Council of the Netherlands, 2005), we divided our respondents into two groups based on their answer to the question: Have you consumed alcohol since you knew you were pregnant, even if you had only one sip? $(0=$ no; $1=$ yes $)$

\section{Questionnaire for Partners}

Partner demographics were measured by the same four items used to assess the pregnant women's demographics (age, education, income and marital status). In addition, partners were asked if this pregnancy was the first he had experienced as a partner $(0=$ no; $1=$ yes $)$.

Partner influence was assessed by the following questions. One item measured the partner's reported norm (I believe that my partner should not drink any alcohol while she is pregnant; $0=$ no; $1=$ yes). The partner's 
average number of standard drinks per day and his average number of drinking days per week were computed by four questions assessing how often the partner used alcohol during weekdays ( 0 = never; $4=$ four days) and weekend days ( $0=$ never; $3=$ three days) in the previous four weeks, and how many standard glasses (equivalent to 10 grams of alcohol) he drank on these respective occasions. The partner's support was measured with one item on a 5-point scale (I support my spouse in not drinking alcoholic beverages during her pregnancy; 1 = no, definitely not; 5 = yes, definitely).

\section{Data Analysis}

Data were analyzed using SPSS 20.0. To assess correlates of prenatal alcohol use, independent sample t-tests and chi-square tests were used to detect differences between pregnant women who did and did not use alcohol. In addition, to assess the relationship between the partner's influence as perceived by the pregnant woman and as reported by her partner, Pearson's correlation analyses for pairs of continuous variables and Spearman's correlation analyses for pairs of an ordinal and a continuous/dichotomous variable were conducted (Field, 2005). Finally, hierarchical logistic regression analyses were conducted. To assess the relative impact of perceived and reported partner influence next to other cognitive factors as derived from the I-Change Model in explaining alcohol use among pregnant women, we tested three models: (a) a model with all I-Change items, including partner influence as perceived by the pregnant woman; (b) a model with all I-Change items in which the pregnant woman's perception of the partner's influence was replaced by her partner's report of this influence; (c) a model with all I-Change items with both the pregnant woman's perception and her partner's report of his influence included. The items were entered in two steps. In the first step, all items were included except partner influence; the second step included perceived and/or reported partner influence. Because of the exploratory nature of this analysis, we used a backward approach (Field, 2005), whereby in both steps, variables were excluded from the model if the probability was greater than .10 based on the likelihood ratio statistic (LR). 


\section{Results}

\section{Sample Characteristics}

The complete sample consisted of 158 couples. The mean age of the pregnant women was 31 years; the mean age of the partners was 34 years. Half of the sample reported a high educational level, and the majority reported a net income of more than $€ 3500$ (which is at least twice as high as the net medium household income in the Netherlands). More than a quarter of the pregnant women had consumed alcohol while they were aware that they were pregnant. A more detailed description of the overall sample is presented in Table 2.1.

\section{Univariate Analyses of Correlates of Prenatal Alcohol Use}

Table 2.2 lists the results of the chi-square tests and T-tests concerning the correlates of prenatal alcohol use, including perceived and reported partner norm, modeling and support. The results indicated that pregnant women using alcohol perceived that their partner considered alcohol abstinence important less often than pregnant women not drinking alcohol. Additionally, pregnant women using alcohol perceived that their partner drank alcohol in their presence more often when compared to pregnant women abstaining from alcohol. Compared to partners of pregnant women abstaining from alcohol, partners of pregnant women using alcohol reported that they believed that their pregnant spouse should abstain from alcohol less often, that they were using alcohol on more days per week; and that they provided a lower amount of support.

Pregnant women using alcohol were older, had a higher education, had less often experienced complications in a previous pregnancy, and had been drinking more drinks per day and reported more drinking days per week before pregnancy when compared to pregnant women who abstained from alcohol. Moreover, pregnant women using alcohol had less often received a midwife's advice to abstain from alcohol; had 
less knowledge of the negative consequences of prenatal alcohol use; underestimated the likelihood and severity of the risks associated with prenatal alcohol use; and perceived fewer pros and more cons concerning alcohol abstinence during pregnancy.

Table 2.1 Characteristics of sample

\begin{tabular}{|c|c|c|c|c|}
\hline & \multicolumn{2}{|c|}{ Pregnant women } & \multicolumn{2}{|l|}{ Partners } \\
\hline & $\mathrm{N}=158$ & & $\mathrm{~N}=158$ & \\
\hline & $\%$ & Mean (SD) & $\%$ & Mean (SD) \\
\hline Age & & $30.7(4.6)$ & & $33.5(5.9)$ \\
\hline \multicolumn{5}{|l|}{ Education: } \\
\hline $\begin{array}{l}\text { Primary school/basic } \\
\text { vocational school }\end{array}$ & 3.8 & & 10.1 & \\
\hline $\begin{array}{l}\text { Secondary vocational } \\
\text { school/high school } \\
\text { degree }\end{array}$ & 37.3 & & 39.2 & \\
\hline $\begin{array}{l}\text { Higher vocational } \\
\text { school/college } \\
\text { degree/university } \\
\text { degree }\end{array}$ & 58.9 & & 48.7 & \\
\hline Missing & 0 & & 1.9 & \\
\hline \multicolumn{5}{|l|}{ Income: } \\
\hline Low income & 2.5 & & 1.9 & \\
\hline Medium income & 38.0 & & 36.1 & \\
\hline High income & 46.8 & & 50.6 & \\
\hline Missing & 12.7 & & 11.4 & \\
\hline $\begin{array}{l}\text { Marital status: Married (or officially } \\
\text { registered) }\end{array}$ & 62.7 & & 60.1 & \\
\hline Percentage first pregnancy: & 58.2 & & 57.6 & \\
\hline Number of weeks pregnant* & & $27.6(9.8)$ & & \\
\hline \multicolumn{5}{|l|}{ Complications in a previous pregnancy*: } \\
\hline Not applicable & 57.6 & & & \\
\hline Yes & 16.5 & & & \\
\hline No & 25.9 & & & \\
\hline Drinks during pregnancy* & 27.8 & & & \\
\hline Drank alcohol before pregnancy* & 91.1 & & & \\
\hline Smokes during pregnancy* & 7.6 & & & \\
\hline Smoked before pregnancy* & 17.2 & & & \\
\hline
\end{tabular}

* partners had not received these questions 
Table 2.2 Univariate analyses of correlates of prenatal alcohol use ( $\mathrm{N}=158)$

\begin{tabular}{|c|c|c|c|c|c|c|c|}
\hline & & \multicolumn{2}{|c|}{$\begin{array}{l}\text { Pregnant women } \\
\text { not using alcohol } \\
\qquad(\mathrm{N}=44)\end{array}$} & \multicolumn{2}{|c|}{$\begin{array}{l}\text { Pregnant women } \\
\text { using alcohol } \\
(\mathrm{N}=114)\end{array}$} & \multirow{2}{*}{$\begin{array}{l}\text { Chi- } \\
\text { square- } \\
\text { test } \\
\chi^{2}\end{array}$} & \multirow{2}{*}{$\begin{array}{l}\text { T-test } \\
\mathrm{T} \\
\end{array}$} \\
\hline & & $\%$ & Mean (SD) & $\%$ & Mean (SD) & & \\
\hline \multirow{10}{*}{$\begin{array}{l}\text { Pre-disposing } \\
\text { factors }\end{array}$} & Age & & $30.21(4.63)$ & & $31.84(4.22)$ & & -2.03 \\
\hline & Education & & $2.47(0.60)$ & & $2.75(0.44)$ & & 3.19 \\
\hline & Income & & $2.46(0.56)$ & & $2.61(0.54)$ & & 1.41 \\
\hline & Married & 65.8 & & 54.5 & & 1.72 & \\
\hline & First pregnancy & 54.4 & & 68.2 & & 2.48 & \\
\hline & $\begin{array}{l}\text { Complications } \\
\text { previous } \\
\text { pregnancy }\end{array}$ & 20.18 & & 6.82 & & $4.12 *$ & \\
\hline & $\begin{array}{l}\text { Drinks per } \\
\text { day before } \\
\text { pregnancy }\end{array}$ & & $0.36(0.76)$ & & $0.75(0.99)$ & & $\begin{array}{l}-2.36 \\
*\end{array}$ \\
\hline & $\begin{array}{l}\text { Drinking days } \\
\text { per week before } \\
\text { pregnancy }\end{array}$ & & $1.06(1.48)$ & & $2.34(2.06)$ & & $\begin{array}{l}-3.77 \\
* * * *\end{array}$ \\
\hline & $\begin{array}{l}\text { Smokes during } \\
\text { pregnancy }\end{array}$ & 9.6 & & 2.3 & & 2.46 & \\
\hline & $\begin{array}{l}\text { Smoked before } \\
\text { pregnancy }\end{array}$ & 19.3 & & 11.6 & & 1.29 & \\
\hline $\begin{array}{l}\text { Information } \\
\text { factors }\end{array}$ & $\begin{array}{l}\text { Received } \\
\text { midwife's advice } \\
\text { not to drink }\end{array}$ & 57.9 & & 38.6 & & $4.72^{*}$ & \\
\hline \multirow[t]{3}{*}{$\begin{array}{l}\text { Awareness } \\
\text { factors }\end{array}$} & Knowledge & & $5.38(1.55)$ & & $4.64(1.86)$ & & $\begin{array}{l}2.54 \\
*\end{array}$ \\
\hline & $\begin{array}{l}\text { Perceived } \\
\text { likelihood }\end{array}$ & & $2.45(1.34)$ & & $1.61(1.10)$ & & $\begin{array}{l}4.00 \\
* * * *\end{array}$ \\
\hline & $\begin{array}{l}\text { Perceived } \\
\text { severity }\end{array}$ & & $2.79(1.19)$ & & $1.77(0.99)$ & & $\underset{* * *}{5.47}$ \\
\hline
\end{tabular}


Table 2.2 (continued). Univariate analyses of correlates of prenatal alcohol use ( $\mathrm{N}=158)$

\begin{tabular}{|c|c|c|c|c|c|c|c|}
\hline & & \multicolumn{2}{|c|}{$\begin{array}{l}\text { Pregnant women } \\
\text { not using alcohol } \\
\qquad(\mathrm{N}=44)\end{array}$} & \multicolumn{2}{|c|}{$\begin{array}{l}\text { Pregnant women } \\
\text { using alcohol } \\
(\mathrm{N}=114)\end{array}$} & \multirow{2}{*}{$\begin{array}{c}\begin{array}{c}\text { Chi- } \\
\text { square- } \\
\text { test }\end{array} \\
\chi^{2}\end{array}$} & \multirow{2}{*}{$\begin{array}{l}\text { T-test } \\
\mathrm{T} \\
\end{array}$} \\
\hline & & $\%$ & Mean (SD) & $\%$ & Mean (SD) & & \\
\hline $\begin{array}{l}\text { Motivational } \\
\text { factors: }\end{array}$ & Pros & & $4.26(0.58)$ & & $3.69(0.95)$ & & 3.70 \\
\hline Attitude & Cons & & $1.46(0.51)$ & & $1.68(0.69)$ & & -1.92 \\
\hline \multirow[t]{2}{*}{ Self efficacy } & $\begin{array}{l}\text { Social Self } \\
\text { Efficacy }\end{array}$ & & $1.79(0.84)$ & & $2.01(0.98)$ & & -1.44 \\
\hline & $\begin{array}{l}\text { Stress Self } \\
\text { Efficacy }\end{array}$ & & $1.44(0.74)$ & & $1.26(0.42)$ & & 1.83 \\
\hline \multirow{3}{*}{$\begin{array}{l}\text { Perceived } \\
\text { partner's } \\
\text { influence }\end{array}$} & $\begin{array}{l}\text { Perceived } \\
\text { partner's norm }\end{array}$ & & $4.42(0.96)$ & & $3.36(1.37)$ & & $\underset{* * * *}{4.71}$ \\
\hline & $\begin{array}{l}\text { Perceived } \\
\text { partner's } \\
\text { modeling }\end{array}$ & & $2.15(1.16)$ & & $1.73(0.97)$ & & $\begin{array}{l}2.14 \\
*\end{array}$ \\
\hline & $\begin{array}{l}\text { Perceived } \\
\text { partner's } \\
\text { support }\end{array}$ & & $3.94(1.20)$ & & $3.86(1.03)$ & & 0.37 \\
\hline \multirow[t]{4}{*}{$\begin{array}{l}\text { Reported } \\
\text { partner's } \\
\text { influence }\end{array}$} & $\begin{array}{l}\text { Reported } \\
\text { partner's norm } \\
\text { (no alcohol in } \\
\text { pregnancy) }\end{array}$ & 82.5 & & 54.5 & & $\underset{* * *}{13.08}$ & \\
\hline & $\begin{array}{l}\text { Reported } \\
\text { partner's } \\
\text { drinks per day }\end{array}$ & & $0.78(0.64)$ & & $0.92(0.82)$ & & -1.09 \\
\hline & $\begin{array}{l}\text { Reported } \\
\text { partner's } \\
\text { drinking days } \\
\text { per week }\end{array}$ & & $2.21(1.95)$ & & $2.98(2.06)$ & & -2.17 \\
\hline & $\begin{array}{l}\text { Reported } \\
\text { partner's } \\
\text { support }\end{array}$ & & $4.26(1.03)$ & & $3.82(1.04)$ & & 2.43 \\
\hline
\end{tabular}

$* \mathrm{p}<0.05 ; * * \mathrm{p}<0.01 ; * * * \mathrm{p}<.001$

\section{Correlation Analyses of Partner's Influence}

Table 2.3 lists the results of the Pearson's and Spearman's correlation analyses. These analyses indicated that the pregnant woman's perception corresponded to her partner's report of his norm, modelling and support. Specifically, the pregnant woman's perception of her partner's norm was significantly positively correlated to her partner's report of his norm. Moreover, the pregnant woman's perception of her partner's alcohol abstinence during her pregnancy had a significant negative correlation with the partner's report of his drinks per day and drinking days per week. 
Finally, the pregnant women's perception of her partner's support was significantly positively correlated to her partner's report of his support. In addition, the pregnant woman's perceived norm related significantly to her partner's reported drinking days per week and support, and her perceived support also related significantly to her partner's reported norm.

Table 2.3 Bivariate correlations of perceived and reported partner's norm, modeling and support $(\mathrm{N}=158)$

\begin{tabular}{lllll}
\hline & & \multicolumn{2}{c}{ Perceived partner's influence } \\
\cline { 3 - 5 } Reported & Norm & Norm & Modeling & Support \\
partner's influence & Partner's drinks per day & $-.58^{* *}$ & .09 & $.22^{* *}$ \\
& $\begin{array}{l}\text { Partner's drinking days per } \\
\text { week }\end{array}$ & $-.20^{*}$ & $-.42^{* *}$ & -.06 \\
& Support & $.32^{* *}$ & $-.48^{* *}$ & -.15 \\
& & & .14 & $.19 *$ \\
\hline
\end{tabular}

$* \mathrm{p}<0.05 ; * * \mathrm{p}<0.01$.

\section{Multivariate Analysis of Prenatal Alcohol Use}

The first step of the models demonstrated that pregnant women were more likely to use alcohol when they had a higher education; were accustomed to drinking on more drinking days per week before their pregnancy; perceived lower severity of harm due to prenatal alcohol use; and perceived fewer pros associated with prenatal alcohol abstinence. However, drinking days per week before pregnancy and pros lost their significance when perceived partner influence was added in the second step of the first model. This first model demonstrated that perceived partner norm was a significant correlate, meaning that pregnant women were more likely to use alcohol when their partner did not consider alcohol abstinence important. In the second step of the second model, which included reported rather than perceived partner influence, reported partner norm was a significant correlate of prenatal alcohol use, whereas pros were no longer significant. In other words, pregnant women were more likely to use alcohol when they had a partner who had reported that he did not believe she should abstain from alcohol during pregnancy. The final model, including both perceived and reported partner influence, 
revealed the same significant correlates as the first model, uncovering the independent influence of perceived partner norm, in addition to education and perceived severity, in explaining prenatal alcohol use. Reported partner influence was not significant. The three models are presented in Table 2.4 .

\section{Discussion}

This study aimed to investigate the influence of partners on pregnant women's alcohol consumption within the context of other factors. Prenatal alcohol use was examined in a dichotomous manner (yes/no), because research has shown that even light alcohol use may pose a risk to the fetus (Nathanson, et al., 2007), and many countries recommend complete abstinence from alcohol (O'Leary, et al., 2007). This study is the first to demonstrate that partner norm as perceived by a pregnant woman is the most important of the perceived and reported constructs of partner influence in explaining prenatal alcohol use, demonstrating that a woman is more likely to use alcohol during pregnancy when she thinks that her partner agrees with this decision.

This study also assessed whether the constructs of perceived and reported partner influence were independently related to prenatal alcohol use. To the best of our knowledge, this study is the first to demonstrate that women who used alcohol during pregnancy more often had a partner whom they perceived and who himself reported that he did not consider prenatal alcohol abstinence important. The present study replicated other research demonstrating that a partner's alcohol use as perceived by the pregnant woman (Bakhireva, et al., 2011) and reported by the partner (Waterson, et al., 1990) is positively related to prenatal alcohol use. Finally, and to the best of our knowledge, this study is the first to demonstrate that partner support as reported by the partner rather than as perceived by the pregnant woman had a significant negative association with prenatal alcohol use. 
Partner's influences and other correlates of prenatal alcohol use

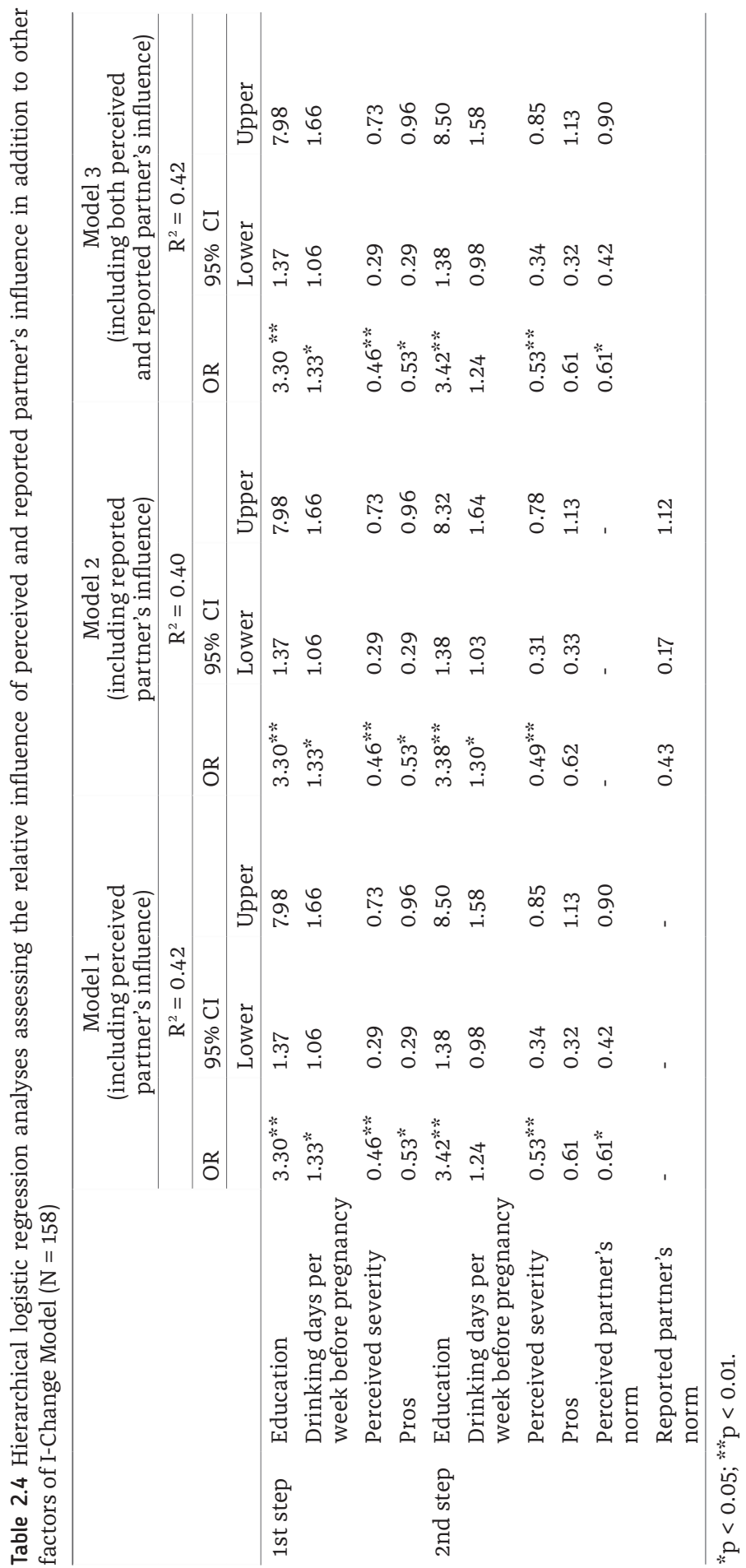


Altogether, the present study demonstrates that prenatal alcohol use is associated with various constructs of partner influence (norm, modeling and support), and it also demonstrates that the strength of the association is dependent upon how the pregnant woman perceives her partner's influence, and what the partner reports regarding his influence. Future research is thus recommended to carefully define which construct of partner influence is being studied and clearly differentiates between the perception of the pregnant woman and the report of the partner.

In the present research, we examined how a pregnant woman's perception related to her partner's report of his influence. We found a relatively strong relationship between perceived and reported partner norm. Apparently, pregnant women are relatively accurate about how much importance their partner attaches to their abstinence from alcohol during pregnancy. The strong relationship between perceived and reported partner modeling indicates strong agreement among couples regarding alcohol use of the partner during the woman's pregnancy. However, we found only a weak relationship between perceived and reported social support. In related research on social support, McNamara and colleagues (2006) explain the discrepancy in their study between perceived and reported social support as representative of a disconnnect between what pregnant women need and what partners think they are providing in terms of support. Altogether, our results further corroborate studies that rely on the perception of the pregnant woman with regard to her partner's norm or modeling. However, caution should be exercised with regard to perceived partner support.

In addition to partner influence, this study investigated other cognitive factors related to prenatal alcohol use. Previous research supports our findings from the multivariate analyses that pregnant women who used alcohol had a higher education (Perham-Hester \& Gessner, 1997), used more alcohol before pregnancy (Chang, et al., 2007), perceived a lower risk of prenatal alcohol use (Testa \& Reifman, 1996) and had a more positive attitude toward prenatal alcohol use (Peadon, et al., 2011) when compared to pregnant women who abstained from alcohol. We could not confirm that pregnant women who used alcohol more often 
also smoked cigarettes (Alvik, et al., 2006; Comasco, et al., 2012). Our non-significant finding is supported by research demonstrating that prenatal smoking is associated with a lower socioeconomic status (Lumley, et al., 2009), whereas prenatal alcohol use is associated with a higher socioeconomic status (Perham-Hester \& Gessner, 1997; Waterson, et al., 1990).

Finally, the correlates of prenatal alcohol use found in the present Dutch study are generally consistent with the correlates found in other studies with Western populations (Perham-Hester, \& Gessner, 1997; Chang, et al., 2007; Testa, \& Reifman, 1996; Peadon, et al., 2011) and might therefore be valid in other countries as well.

\section{Strengths and Limitations}

This study has several strengths. This study is the first to differentiate between the three constructs of partner influence and to relate partner influence to prenatal alcohol use. Moreover, by studying the correlation between a pregnant woman's perception and her partner's report of his influence, this study demonstrated which constructs we can rely on the pregnant woman's perception for interpretation and which constructs also require the partner's report for interpretation. Still, uncertainty remains as to whether either the pregnant woman's perception or the partner's report reflects actual behavior. Finally, because we found that partner norm influences prenatal alcohol use, these findings suggest the importance of addressing both pregnant women and their partners in prenatal alcohol prevention programs.

This study is also subject to limitations. First, this study used a convenience sample of couples who were recruited by midwives. Thus, not all midwives may have been willing to participate, and participating midwives may have selected couples to invite for participation. As such, there may have been participation bias among those couples willing to participate. Indeed, the relatively high levels of education and income confirm participation bias, and unfortunately due to the 
lack of a representative population, the results of this study cannot be generalized. However, a participation bias does not diminish the results of this study, because the whole range of the studied factors was found in our sample, allowing the investigation of these variables as correlates of prenatal alcohol use. Second, the study was of a crosssectional nature. Therefore, we were unable to draw conclusions about the causality of the associations found. Third, our study addressed social norms, modeling and support of partners as they relate to alcohol consumption in pregnant women. Yet, other social influences also exist that may have an influence on a pregnant woman's decision regarding alcohol consumption, such as concordant or discordant drinking habits (e.g. Torvik, et al., 2013) and intimate partner violence (e.g. Devries, et al., 2014). Our study did not address these issues, and more research may be needed, particularly in subgroups of women who experience partner violence, to study the influence of these factors. Finally, throughout the paper, we regarded the partners as male. However, we did not actually assess the gender of the partner, and we cannot be certain that all of the partners were male. Thus, we cannot differentiate between the influence of male and female partners on the pregnant women, nor can we differentiate between partners who were or were not the biological fathers of the respective pregnant women's babies.

\section{Conclusions}

Because no safe level of alcohol consumption has been determined and many pregnant women still use alcohol, effective interventions are needed to prevent prenatal alcohol use. The present study contributed to this development by identifying important correlates of prenatal alcohol use, with special attention given to partner influence, both as perceived by the pregnant woman and reported by the partner. Hence, the effectiveness of interventions can be improved when such interventions address both the pregnant woman and her partner. 



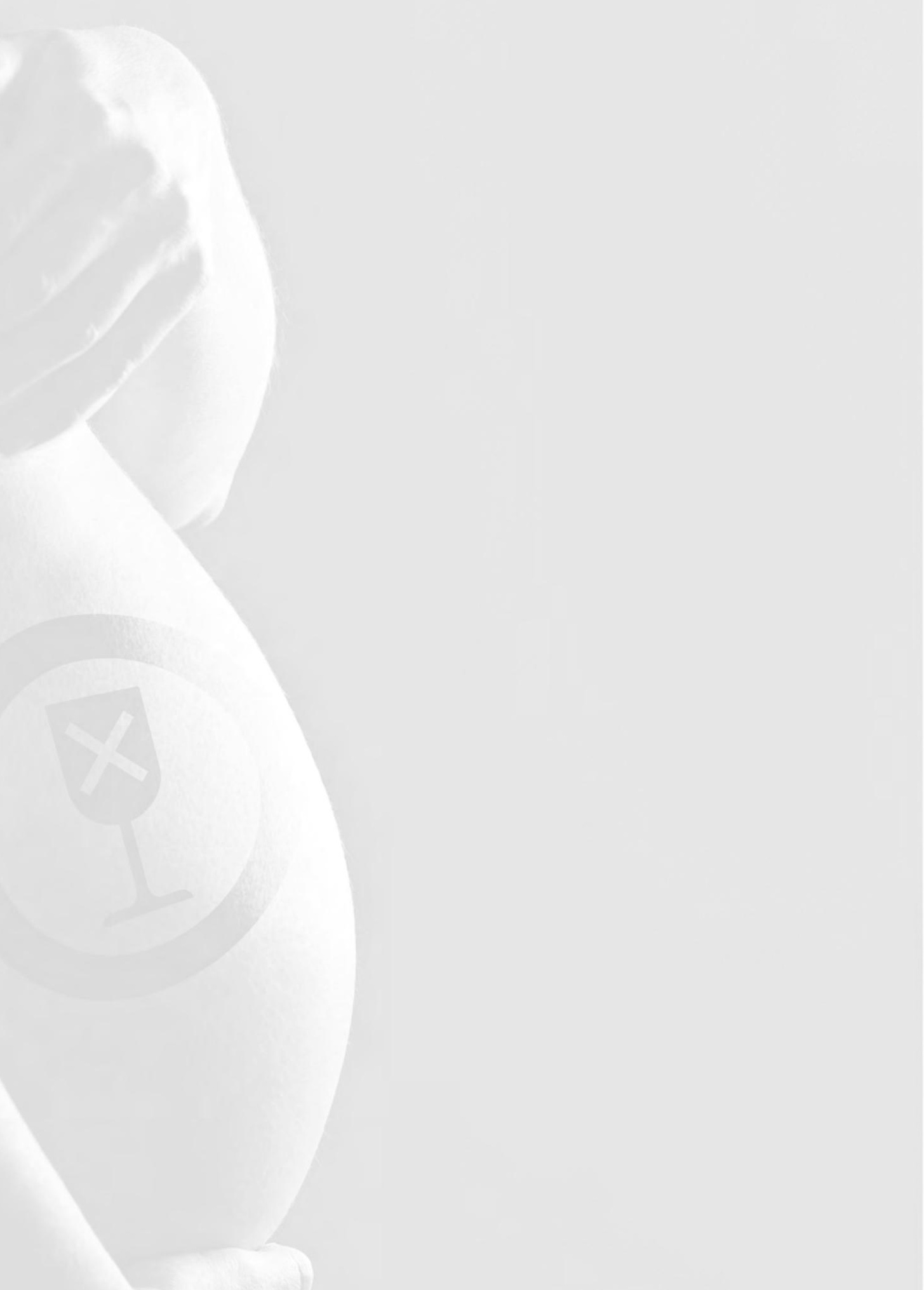




\section{CHAPTER 3}

\section{Correlates of partner support to abstain from prenatal alcohol use: A cross-sectional survey among Dutch partners of pregnant women}

Published as: Van der Wulp, N.Y., Hoving, C., de Vries, H. (2015). Correlates of partner support to abstain from prenatal alcohol use: A cross-sectional survey among Dutch partners of pregnant women. Health and Social Care in the Community. 


\section{Abstract}

Background: Partners can play an important role, but are often ignored in interventions targeting the prevention of prenatal alcohol use. A better understanding of the correlates of partner support to abstain from prenatal alcohol use can help to make a better use of partner support. Objectives: The aim of this study was to identify correlates of this support by analysing differences between partners reporting low versus high support.

Methods: An online cross-sectional study among 237 Dutch partners of pregnant women was conducted. Respondents were recruited through Dutch midwifery practices in September-October 2009. Questionnaires were based on the I-Change Model.

Results: Chi-square and t-tests showed that partners reporting high support were more likely to desire their partner to abstain from alcohol use and to have received advice from their pregnant spouse or midwife that abstinence was desirable. They also had stronger perceptions that the baby would experience harm from prenatal alcohol use and that harm could be more severe, and they saw more advantages and fewer disadvantages of providing support. They also reported more influence from their social environment encouraging their support, had greater self-efficacy and had a stronger intention to support their partner during the remainder of the pregnancy compared to partners reporting low support.

Conclusions: Health professionals may improve their alcohol advice by discussing the advantages and disadvantages of support with the partner and by encouraging couples to discuss and propose solutions for the situations in which partners find it difficult not to support alcohol abstinence. By providing insight on important correlates of partner support, this study expands the research area aiming to reduce prenatal alcohol use. 


\section{Introduction}

Alcohol use by pregnant women is one of the leading preventable causes of damage to the unborn child (Centers for Disease Control and Prevention, 2002). Several psychological and educational interventions have been developed to prevent prenatal alcohol use (Stade, et al., 2009). In line with many cognitive behavioural models (Ajzen, 1985; Janz \& Becker, 1984; De Vries, et al., 2003) important cognitive factors have been taken into account in these interventions, such as attitude towards abstinence and self-efficacy (Reynolds, et al., 1995; Chang, et al., 1999). However, despite the importance attached to social influence in determining health behaviour (Ajzen, 1985; De Vries, et al., 1995), partner support has thus far received relatively limited attention in these intervention studies.

There is sufficient evidence for the effect of partner support on a pregnant woman's behaviour. For example, research on smoking during pregnancy has shown that partner support has a positive effect on smoking cessation during pregnancy (Ginsberg, Hall, \& Rosinski, 1991; McBride, et al., 2004; Koshy, et al., 2010). Furthermore, research on breastfeeding has consistently identified fathers as an important source of support in the decision to breast-feed and its implementation (Wolfberg, et al., 2004; Tarrant, et al., 2010). With regard to prenatal alcohol use, research has also shown that partner support affects a pregnant woman's drinking behaviour (Ockene, et al., 2002). One randomised trial showed the effectiveness of a patient-partner intervention on prenatal alcohol consumption for heavy drinkers (Chang, et al., 2005). These studies imply that prenatal alcohol use can more effectively be prevented when partner support is incorporated in these interventions. This requires that researchers are aware of how to increase partner support. Nevertheless, no study has thus far been conducted on the correlates of partner support to abstain from prenatal alcohol.

This study was designed to fill this gap and aimed to identify the correlates of partner support to abstain from prenatal alcohol. The Integrated Model for Motivational and Behavioural Change (I-Change 
Model; De Vries, et al., 2003) provided the theoretical framework for this study. The I-Change Model combines several socio-cognitive behavioural models (Prochaska \& DiClemente, 1983; Janz \& Becker, 1984; Ajzen 1985) and has been used effectively to assess important determinants of a diversity of health behaviours (e.g. De Vries, et al., 2006b; Hoving, Mudde \& De Vries, 2006a; E.S. Smit, De Vries, \& Hoving, 2013a). The I-Change Model posits that a person's behaviour is the result of intentions and abilities. The motivational factors attitudes, social influences (De Vries, et al., 1994) and self-efficacy (Bandura, et al., 1988; De Vries, Dijkstra, \& Kuhlman, 1988) determine the level of intention to execute a certain health behaviour, or in this case providing support to the pregnant spouse to abstain from alcohol use. The motivational factors are in turn determined by various predisposing factors (e.g. demographics and behavioural factors), information factors (e.g. messages and sources) and awareness factors (e.g. knowledge and risk perceptions).

This is the first study aiming to identify the correlates of partner support to abstain from prenatal alcohol use. The explorative nature demands that this study amplifies the differences in support between partners. We therefore tested differences between partners who were low versus high in partner support with regard to factors of the I-Change Model. Specifically, we tested whether partners low versus high in partner support differed with regard to predisposing (i.e. demographics, drinking behaviour and partner's desire that his spouse abstains from alcohol use), information (i.e. advice about prenatal alcohol use and support), awareness (i.e. knowledge and risk perceptions), motivational factors (i.e. perceived pros and cons, social influences and self-efficacy) and intention. 


\section{Methods}

\section{Procedure}

Respondents of this cross-sectional study were partners of pregnant women, recruited through Dutch midwifery practices in SeptemberOctober 2009. Midwives are important care providers for pregnant women and their partner as $75 \%$ of the Dutch women at the beginning of their pregnancy and 36\% of the Dutch women throughout their entire pregnancy receive care only from a midwife (Wiegers, Van der Velden, \& Hingstman, 2005). All midwifery practices in the Netherlands (approximately 500) were asked to distribute flyers among their clients' partners with information about the purpose of the study and a link to an online questionnaire developed with Tailorbuilder software (OSE, the Netherlands). Each flyer contained an informational section plus a login-code to gain access to the online study questionnaire.

After three weeks a reminder email was sent to all midwifery practices. The questionnaires were filled in anonymously; the name of the respondent as well as the name of the attending midwifery practice were not collected. Ethics approval for this study was granted by a human research ethics committee (METC Atrium MC - Maaslandziekenhuis; 08-N-50).

\section{Questionnaire}

The online questionnaire consisted of 115 questions and was a modification of questionnaires used in previous studies applying the I-Change Model (Dijkstra, De Vries, \& Bakker, 1996; Dijkstra, De Vries, \& Royackers, 1998a; Hoving, Mudde, \& De Vries, 2007a).

The first set of questions assessed predisposing factors, which included demographics, drinking behaviour and partner's desire that his spouse abstains from alcohol use. Demographics were assessed with three items: age, education $(1=$ primary school/basic vocational school; 
2 = secondary vocational school/high school degree; 3 = higher vocational school/college degree/university degree), and income $(1=0.5$ or less; $2=$ $0.5-2 ; 3=$ more than 2 times net Dutch median household income). Partners' drinking behaviour was measured by asking respondents whether they have ever used alcohol ( $0=$ no; $1=$ yes). Whether the partner desired that his pregnant spouse abstains from alcohol use was measured by one item $(0=$ no, small amounts/regular amounts of alcohol is okay/no opinion, don't know; $1=$ yes, abstinence desired).

The next set of questions assessed information factors. Specifically, four questions assessed whether the respondent had received advice about prenatal alcohol use and support. The partner was asked whether he had received advice (a) from the pregnant spouse that abstinence by pregnant women from prenatal alcohol use is desirable; (b) advice from the midwife that abstinence by pregnant women from prenatal alcohol use is desirable; (c) advice from the pregnant spouse that support to abstain from alcohol use is needed; and (d) advice from the midwife that support to abstain from alcohol use is needed ( $0=$ no advice, different advice, don't know, $1=y e s$, received this advice).

Awareness factors were assessed with questions about knowledge and risk perceptions. Knowledge was measured by an index of nine statements ( $0=$ false/don't know, $1=$ true) which assessed whether participants were aware about the harmfulness of drinking alcohol during pregnancy (e.g. alcohol use during pregnancy can lead to brain damage of the unborn child). An index of total knowledge was calculated by adding the number of items which were correctly answered. Risk perception was assessed with two items, that is perceived likelihood that the baby would experience harm ( 1 = very low; 5 = very high) and perceived severity of that harm to the baby $(1=$ not serious; $5=$ very serious) if the pregnant woman would drink one glass of alcohol per month.

The subsequent set of questions assessed motivational factors, which included attitudes, social influences and self-efficacy. Attitudes towards providing support to the pregnant partner to abstain from alcohol use 
were assessed with 10 items measured on a 5-point Likert scale ( 1 = fully disagree, 5 = fully agree). Five questions measured pros or advantages (e.g. If I support my pregnant partner to abstain from alcohol during pregnancy, my partner will appreciate that; $\alpha=0.84$ ) and five questions measured cons or disadvantages (e.g. If I support my partner to abstain from alcohol during pregnancy, my partner will get irritated; $\alpha=0.86$ ) of providing support. Social influences with regard to providing support were assessed with six items on a 5-point scale ( 1 = fully disagree; 5 = fully agree) concerning norms (My friends/family consider(s) it important that I support my pregnant partner not to drink alcohol; $\alpha=$ 0.95), modelling (My friends/family support(s) their partner to abstain from alcohol during pregnancy; $\alpha=0.93$ ) and encouragement (My friends/family encourage(s) me to support my partner to abstain from alcohol during pregnancy; $\alpha=0.94)$. Self-efficacy was measured by eight items asking respondents to indicate on a 5-point scale ( 1 = very difficult; $5=$ not difficult at all) their difficulty with supporting their partner to abstain from alcohol in certain situations (e.g. When my partner and I have something to celebrate; $\alpha=0.94$ ).

Intention was assessed with one item asking respondents to indicate on a 5 -point scale ( $1=$ no, definitely not; $5=$ yes, definitely) if they had the intention to support their partner not to drink alcohol during the remainder of the pregnancy.

The outcome variable was partner support to abstain from alcohol during pregnancy. This variable was assessed by one item: I support my spouse not to drink alcoholic beverages during her pregnancy $(1=$ no, definitely not; 5 = yes, definitely).

\section{Data analysis}

Data were exported from the Tailorbuilder programme and analysed using SPSS 19.0. Descriptive statistics of sample characteristics were estimated as frequencies and percentages or means and standard deviations as considered appropriate. Before analysing the correlates, 
the variable of partner support to abstain from alcohol was carefully examined. With MPlus 6.11, a latent class analysis (Collins \& Lanza, 2010) was conducted to make a well-founded, data-driven decision on the number of groups that were most parsimonious and descriptive of the data. Several statistical indicators were used, including the Bayesian Information Criterion (BIC), the Lo-Mendell-Rubin likelihood ratio test (LMR-LRT), and entropy (Nagin, 2005). The LMR-LRT showed a significantly better fit with two groups than with one, but not a significantly better fit with three groups than with two, supported by the other indicators. The group low in partner support consisted of participants who had scored 1, 2 or 3 on the 5-point scale; the group high in partner support consisted of participants who had scored 4 or 5. Statistical differences between respondents low and high in partner support to abstain from alcohol during pregnancy with regard to predisposing, information, awareness, motivational factors and intention were analysed using chi-square test for dichotomous data, the chi-square test for trend for ordinal data and the t-test for interval data, as appropriate. We followed Norman (2010) in reporting the results of t-tests to compare the 5-point Likert scale variables between the two groups. Rerunning the analyses with chi-square tests for trend revealed that the results did not differ noteworthy from the results of the t-tests.

\section{Results}

\section{Sample characteristics}

The total sample consisted of 237 partners. The age of respondents varied from 20 to 54 years, with a mean of 33.35 (SD = 5.59; see Table 3.1). Half of the sample reported a high educational level, the majority had an income more than twice the net Dutch medium household income ( $€ 1700$ per month) or higher. Respondents reported a mean partner support of 4.14 $(S D=1.03)$, indicating that on average respondents greatly supported their pregnant partner to abstain from alcohol. According to the latent class analysis, respondents could be divided in one group low in partner 
support, consisting of 64 participants $(27.0 \%)$ and one group high in partner support, consisting of 173 participants (73.0\%).

\section{Differences in predisposing, information and awareness factors}

Predisposing, information and awareness factors were compared between respondents providing low partner support and those providing high partner support. There were no significant differences with regard to demographic characteristics or drinking behaviour. However, respondents reporting high support significantly more often desired their pregnant spouse to abstain from alcohol use compared to respondents low in partner support (high 85.9\%; low 57.1\%).

With regard to information factors, respondents reporting high support had more often received advice either from their pregnant spouse (high $71.7 \%$; low $56.2 \%$ ) or from the midwife (high $61.8 \%$; low $43.8 \%$ ) that abstinence from prenatal alcohol use was desirable compared to respondents reporting low support. In addition, the first group had more often received advice from the midwife that support to abstain from prenatal alcohol use was needed (high 12.1\%; low 1.6\%).

Respondents reporting high support perceived it being more likely that the baby would experience harm from one glass of prenatal alcohol per month (high $\mathrm{M}=2.29$; low $\mathrm{M}=1.67$ ) and perceived this harm to be more serious (high $M=2.55$; low $M=1.94$ ) compared to respondents reporting low support. The two groups did not significantly differ with regard to their knowledge about the harmfulness of drinking during pregnancy.

\section{Differences in motivational factors and intention}

Respondents reporting high versus low partner support also differed significantly from each other with regard to motivational factors (see Table 3.2). 
Table 3.1 Differences in predisposing, information and awareness factors between respondents low $(\mathrm{N}=64)$ versus high $(\mathrm{N}=173)$ in partner support

\begin{tabular}{|c|c|c|c|c|c|c|}
\hline \multirow[t]{2}{*}{ Characteristic } & \multirow[t]{2}{*}{ Overall } & Partner sup & port & \multirow{2}{*}{$\begin{array}{l}\text { Chi- } \\
\text { square } \\
\text { test } \\
\chi^{2}\end{array}$} & \multirow{2}{*}{$\begin{array}{l}\text { Chi- } \\
\text { square } \\
\text { test for } \\
\text { trend } \\
\chi_{\text {TREND }}^{2}\end{array}$} & \multirow{2}{*}{$\begin{array}{l}\text { t-test } \\
\mathrm{T}\end{array}$} \\
\hline & & Low & High & & & \\
\hline \multicolumn{7}{|l|}{ Partner support } \\
\hline $\begin{array}{l}\text { I support my spouse } \\
\text { not to drink } \\
\text { alcoholic beverages } \\
\text { during her } \\
\text { pregnancy; Mean } \\
\text { (SD) }\end{array}$ & $4.14(1.03)$ & $2.67(0.59)$ & $4.68(0.47)$ & & & $27.28 * * *$ \\
\hline \multicolumn{7}{|l|}{ Predisposing factors } \\
\hline Mean Age (SD) & $33.35(5.59)$ & $32.83(5.76)$ & $33.54(5.53)$ & & & 0.87 \\
\hline Education: & & & & & 1.25 & \\
\hline $\begin{array}{l}\text { Primary school/basic } \\
\text { vocational school }\end{array}$ & $8.5 \%$ & $4.8 \%$ & $9.9 \%$ & & & \\
\hline $\begin{array}{l}\text { Secondary } \\
\text { vocational } \\
\text { school/high school } \\
\text { degree }\end{array}$ & $41.5 \%$ & $41.3 \%$ & $41.5 \%$ & & & \\
\hline $\begin{array}{l}\text { Higher vocational } \\
\text { school/college } \\
\text { degree/university } \\
\text { degree }\end{array}$ & $50.0 \%$ & $54.0 \%$ & $48.5 \%$ & & & \\
\hline Income: & & & & & 0.04 & \\
\hline $\begin{array}{l}0.5 \text { or less times net } \\
\text { Dutch median } \\
\text { household }\end{array}$ & $0.5 \%$ & $1.7 \%$ & $0 \%$ & & & \\
\hline $\begin{array}{l}0.5-2 \text { times net } \\
\text { Dutch median } \\
\text { household }\end{array}$ & $39.8 \%$ & $36.2 \%$ & $41.2 \%$ & & & \\
\hline $\begin{array}{l}\text { more than } 2 \text { times } \\
\text { net Dutch median } \\
\text { household }\end{array}$ & $59.7 \%$ & $62.1 \%$ & $58.8 \%$ & & & \\
\hline Has used alcohol & $97.5 \%$ & $98.4 \%$ & $97.1 \%$ & 0.33 & & \\
\hline $\begin{array}{l}\text { Desired abstinence of } \\
\text { his pregnant } \\
\text { spouse's alcohol use }\end{array}$ & $78.1 \%$ & $57.1 \%$ & $85.9 \%$ & $22.21 * * *$ & & \\
\hline
\end{tabular}


Table 3.1 (continued). Differences in predisposing, information and awareness factors between respondents low $(\mathrm{N}=64)$ versus high $(\mathrm{N}=173)$ in partner support

\begin{tabular}{|c|c|c|c|c|c|c|}
\hline \multirow[t]{2}{*}{ Characteristic } & \multirow[t]{2}{*}{ Overall } & \multicolumn{2}{|c|}{ Partner support } & \multirow{2}{*}{$\begin{array}{l}\begin{array}{l}\text { Chi- } \\
\text { square } \\
\text { test }\end{array} \\
x^{2}\end{array}$} & \multirow{2}{*}{$\begin{array}{l}\text { Chi- } \\
\text { square } \\
\text { test for } \\
\text { trend } \\
\chi_{\text {TREND }}^{2}\end{array}$} & \multirow{2}{*}{$\begin{array}{l}\text { t-test } \\
\mathrm{t}\end{array}$} \\
\hline & & Low & High & & & \\
\hline \multicolumn{7}{|l|}{ Information factors } \\
\hline $\begin{array}{l}\text { Received advice from } \\
\text { pregnant spouse that } \\
\text { abstinence from } \\
\text { prenatal alcohol use } \\
\text { is desirable }\end{array}$ & $67.5 \%$ & $56.2 \%$ & $71.7 \%$ & $5.07^{*}$ & & \\
\hline $\begin{array}{l}\text { Received advice from } \\
\text { midwife that } \\
\text { abstinence from } \\
\text { prenatal alcohol use } \\
\text { is desirable }\end{array}$ & $57.0 \%$ & $43.8 \%$ & $61.8 \%$ & $6.24^{*}$ & & \\
\hline $\begin{array}{l}\text { Received advice from } \\
\text { pregnant spouse that } \\
\text { support to abstain } \\
\text { from prenatal alcohol } \\
\text { use is needed }\end{array}$ & $6.8 \%$ & $4.7 \%$ & $7.5 \%$ & 0.59 & & \\
\hline $\begin{array}{l}\text { Received advice from } \\
\text { midwife that support } \\
\text { to abstain from } \\
\text { prenatal alcohol use } \\
\text { is needed }\end{array}$ & $9.3 \%$ & $1.6 \%$ & $12.1 \%$ & $6.21^{*}$ & & \\
\hline \multicolumn{7}{|l|}{ Awareness factors } \\
\hline Knowledge & $5.01(1.82)$ & $4.70(1.89)$ & $5.12(1.79)$ & & & 1.57 \\
\hline Perceived likelihood & $2.12(1.30)$ & 1.67 (1.09) & $2.29(1.33)$ & & & $3.65 * * *$ \\
\hline Perceived severity & $2.38(1.21)$ & $1.94(1.10)$ & $2.55(1.21)$ & & & $3.55 * * *$ \\
\hline
\end{tabular}

*p $<0.05 ; *$ p $<0.01 ; * * * \mathrm{p}<.001$

First, respondents reporting high support perceived more advantages (high $\mathrm{M}=4.02$; low $\mathrm{M}=3.28$ ) and fewer disadvantages (high $\mathrm{M}=1.47$; low $M=1.81$ ) of providing support than respondents reporting low support. In addition, the respondents reporting high support were more convinced that partner support was the social norm among friends and family (high $M=4.00$; low $M=2.82$ ), were more exposed to social modelling (high $\mathrm{M}=4.13$; low $\mathrm{M}=3.41$ ) and were more socially encouraged to provide support (high $M=3.26$; low $M=2.60$ ) compared to the respondents reporting low support. Finally, respondents reporting high support had more self-efficacy compared to respondents reporting low support (high $\mathrm{M}=4.26$; low $\mathrm{M}=3.94$ ). This significant difference 
in self-efficacy was largely attributable to the finding that respondents reporting high support found it easier to provide partner support when their partner had not had a drink for a long time compared to respondents reporting low support (high $\mathrm{M}=4.29$; low $\mathrm{M}=3.83$ ).

Table 3.2 Motivational and intentional differences between respondents low $(\mathrm{N}=64)$ versus high $(\mathrm{N}=173)$ in partner support

\begin{tabular}{|c|c|c|c|c|c|}
\hline \multirow[t]{3}{*}{ Characteristic } & \multirow{3}{*}{$\begin{array}{l}\text { Overall } \\
\text { Mean (SD) }\end{array}$} & \multicolumn{2}{|c|}{ Partner support } & \multirow{2}{*}{\multicolumn{2}{|c|}{ t-test }} \\
\hline & & Low & High & & \\
\hline & & Mean (SD) & Mean (SD) & $\mathrm{T}$ & \\
\hline \multicolumn{6}{|l|}{ Attitude } \\
\hline \multicolumn{6}{|l|}{$\begin{array}{l}\text { If I support my pregnant partner to } \\
\text { abstain from alcohol during } \\
\text { pregnancy, then ... }\end{array}$} \\
\hline Advantages & $3.82 \overline{(0.89)}$ & $3.28(0.86)$ & $4.02(0.80)$ & 6.19 & - \\
\hline ... that is better for my child's health & $4.28 \overline{(1.06)}$ & $3.83 \overline{(1.19)}$ & $4.45 \overline{(0.96)}$ & $\begin{array}{l}4.14 \\
* * *\end{array}$ & - \\
\hline ... my partner will appreciate that & $3.91(1.00)$ & $3.36(1.05)$ & $4.12(0.91)$ & $\underset{* * *}{5.46}$ & \\
\hline $\begin{array}{l}\text {... it is easier for my partner not to } \\
\text { drink }\end{array}$ & $3.52(1.23)$ & $3.03(1.20)$ & $3.71(1.20)$ & $\underset{* * *}{3.84}$ & \\
\hline ... I am a good partner & $3.70(1.14)$ & $3.08(1.13)$ & $3.93(1.06)$ & 5.40 & \\
\hline ... I am a good father & $3.66(1.18)$ & $3.08(1.15)$ & $3.88(1.12)$ & $\underset{* * *}{4.85}$ & \\
\hline Disadvantages $\overline{ }$ & $1.56 \overline{(0.67)}$ & $1.81 \overline{(0.75)}$ & $1.47 \overline{(0.61)}$ & $\begin{array}{l}-3.27 \\
* *\end{array}$ & \\
\hline $\begin{array}{l}\text {... my friends will not consider me } \\
\text { cool }\end{array}$ & $1.46 \overline{(0.81)}$ & $1.75 \overline{(1.01)}$ & $1.36 \overline{(0.69)}$ & -2.87 & - \\
\hline ... I will be considered 'patronizing' & $1.64(0.86)$ & $1.97(0.94)$ & $1.51(0.80)$ & $\begin{array}{l}-3.70 \\
* * * *\end{array}$ & \\
\hline ... my partner will get irritated & $1.58(0.82)$ & $1.84(0.93)$ & $1.48(0.75)$ & $\begin{array}{l}-2.81 \\
* *\end{array}$ & \\
\hline ... parties are less fun & $1.61(0.89)$ & $1.77(0.96)$ & $1.55(0.87)$ & -1.66 & \\
\hline ... it will cost me too much energy & $1.52(0.77)$ & $1.72(0.86)$ & $1.45(0.73)$ & $\begin{array}{l}-2.26 \\
*\end{array}$ & \\
\hline \multicolumn{6}{|l|}{ Social Influences } \\
\hline Social norms & $3.71(1.20)$ & $2.82(1.26)$ & $4.00(1.03)$ & $\underset{* * *}{6.64}$ & \\
\hline $\begin{array}{l}\text { My friends consider it important that I } \\
\text { support my pregnant partner not to } \\
\text { drink alcohol }\end{array}$ & $3.63 \overline{(1.27)}$ & $2.78 \overline{(1.27)}$ & $3.94 \overline{(1.12)}$ & $\begin{array}{l}6.07 \\
* * *\end{array}$ & \\
\hline $\begin{array}{l}\text { My family consider it important that I } \\
\text { support my pregnant partner not to } \\
\text { drink alcohol }\end{array}$ & $3.75(1.22)$ & $2.86(1.31)$ & $4.04(1.03)$ & $\underset{* * *}{5.77}$ & \\
\hline
\end{tabular}


Table 3.2 (continued). Motivational and intentional differences between respondents low $(\mathrm{N}=$ 64) versus high $(\mathrm{N}=173)$ in partner support

\begin{tabular}{|c|c|c|c|c|}
\hline \multirow[t]{3}{*}{ Characteristic } & \multirow[t]{2}{*}{ Overall } & \multicolumn{2}{|c|}{ Partner support } & \multirow[t]{2}{*}{ t-test } \\
\hline & & Low & High & \\
\hline & Mean (SD) & Mean (SD) & Mean (SD) & $\mathrm{t}$ \\
\hline Social modeling & $3.97(0.93)$ & $3.41(1.08)$ & $4.13(0.82)$ & 3.88 \\
\hline $\begin{array}{l}\text { My friends support their partner to } \\
\text { abstain from alcohol during } \\
\text { pregnancy }\end{array}$ & $3.95 \overline{(0.98)}$ & $3.43 \overline{(1.12)}$ & $4.10 \overline{(0.88)}$ & $\begin{array}{l}3.35 \\
* *\end{array}$ \\
\hline $\begin{array}{l}\text { My family supports their partner to } \\
\text { abstain from alcohol during pregnancy }\end{array}$ & $4.00(0.95)$ & $3.44(1.10)$ & $4.16(0.84)$ & 3.82 \\
\hline Social encouragement & $3.09(1.18)$ & $2.60(1.23)$ & $3.26(1.11)$ & $\underset{* *}{3.47}$ \\
\hline $\begin{array}{l}\text { My friends encourage me to support } \\
\text { my partner to abstain from alcohol } \\
\text { during pregnancy }\end{array}$ & $3.05 \overline{(1.22)}$ & $2.63 \overline{(1.25)}$ & $3.19 \overline{(1.18)}$ & 2.73 \\
\hline $\begin{array}{l}\text { My family encourages me to support } \\
\text { my partner to abstain from alcohol } \\
\text { during pregnancy }\end{array}$ & $3.14(1.21)$ & $2.63(1.23)$ & $3.32(1.16)$ & 3.52 \\
\hline Self efficacy & $4.18(0.82)$ & $3.94(0.91)$ & $4.26(0.78)$ & $\underset{*}{2.38}$ \\
\hline \multicolumn{5}{|l|}{$\begin{array}{l}\text { I find it difficult to support my partner } \\
\text { to abstain from alcohol during } \\
\text { pregnancy when... }\end{array}$} \\
\hline ... my partner craves for alcohol & $4.04(1.12)$ & $3.76(1.11)$ & $4.13(1.11)$ & 1.88 \\
\hline $\begin{array}{l}\text {... my partner has not had a drink for a } \\
\text { long time }\end{array}$ & $4.19(0.92)$ & $3.83(1.00)$ & $4.29(0.87)$ & 2.89 \\
\hline $\begin{array}{l}\text {... my partner and I are in a café or } \\
\text { restaurant }\end{array}$ & $4.12(1.02)$ & $3.89(1.05)$ & $4.20(1.00)$ & 1.78 \\
\hline $\begin{array}{l}\text {... my partner and I have something to } \\
\text { celebrate }\end{array}$ & $4.02(1.07)$ & $3.79(1.08)$ & $4.10(1.06)$ & 1.73 \\
\hline ... I have an argument with my partner & $4.39(0.79)$ & $4.24(0.93)$ & $4.44(0.73)$ & 1.41 \\
\hline ... I long for a drink myself & $4.16(0.99)$ & $3.96(1.10)$ & $4.23(0.94)$ & 1.66 \\
\hline ... I am stressed & $4.30(0.87)$ & $4.13(0.96)$ & $4.35(0.84)$ & 1.50 \\
\hline ... I am tired & $4.32(0.84)$ & $4.11(0.99)$ & $4.39(0.77)$ & 1.96 \\
\hline Intention & $4.50(1.17)$ & $3.67(1.66)$ & $4.81(0.72)$ & $\begin{array}{l}5.29 \\
* * * x\end{array}$ \\
\hline
\end{tabular}

$* \mathrm{p}<0.05 ; * \mathrm{p}<0.01 ; * * \mathrm{p}<.001$.

Lastly, respondents reporting high support had a stronger intention to support their partner to abstain from alcohol during the remainder of the pregnancy compared to respondents reporting low support (high $\mathrm{M}$ = 4.81; low $\mathrm{M}=3.67$ ). 


\section{Discussion}

In this study, we compared the cognitive factors of partners who were high versus low in partner support. This study showed that partners reporting high support were more likely to desire their partner to abstain from alcohol use and to have received advice from their pregnant spouse or midwife that abstinence was desirable. They also had stronger perceptions that the baby would experience harm from prenatal alcohol use and that harm could be more severe, and they saw more advantages and fewer disadvantages of providing support. They also reported more influence from their social environment encouraging their support, had greater self-efficacy and had a stronger intention to support their partner during the remainder of the pregnancy compared to partners reporting low support.

The significant difference between partners reporting high versus low support with regard to motivational factors and intention is in line with the I-Change Model and other theories explaining health behaviour (e.g. Ajzen, 1985). These findings suggest several intervention points for the involvement of partner support to prevent prenatal alcohol use. First, health professionals may improve their alcohol advice by discussing the advantages and disadvantages of support with the partner. Second, as social norms are important correlates of partner support to abstain from alcohol, governmental organizations may consider to increase public awareness of the risks of alcohol use during pregnancy. Third, previous research (van der Wulp, Hoving, \& De Vries, 2013) has shown that partners are willing to discuss prenatal alcohol use with their pregnant spouse using alcohol. By encouraging couples to discuss the situations in which partners find it difficult not to support alcohol abstinence, health professionals may help to increase partner support. However, with respect to these suggestions, the cross-sectional design of the present study should be remembered. Longitudinal research is needed to confirm that an increase of the motivational factors and intention leads to an increase of partner support to abstain from prenatal alcohol use. 
The present study's finding that partners reporting high versus low support differed with regard to the advice they had received from their midwife is supported by previous research stressing the role of health professionals in preventing prenatal alcohol use (Chang, 2004). The present study showed that partners reporting high support had more often received advice from their midwife that abstinence from prenatal alcohol use is desirable and partner support is needed compared to partners reporting low support. Midwives in the Netherlands (van der Wulp, et al., 2013) as well as in other countries (Holmqvist \& Nilsen, 2010; Jones, et al., 2011; Kesmodel \& Kesmodel, 2011) do not always advise their clients to abstain from alcohol. Moreover, the limited research conducted on midwives' advice to partners of pregnant women shows that midwives largely ignore partners in their alcohol advice (van der Wulp, et al., 2013). Despite the exploratory nature of this study, these findings advocate the involvement of the partner in the midwife's alcohol advice, presenting an extra opportunity to increase prenatal alcohol abstinence.

This study showed that almost half of the partners low in partner support did not desire complete alcohol abstinence from his pregnant spouse. This result is consistent with findings from a qualitative study showing that partners were relatively tolerant towards prenatal alcohol use (van der Wulp, et al., 2013). Due to the scarcity of studies focusing on partners and prenatal alcohol use, it is unclear why partners are tolerable with regard to prenatal alcohol use. The opposite should be expected as his pregnant spouse's alcohol use also affects his child. Perhaps this tolerance results from the fact that in many Western countries, including the Netherlands, the partner has no juridical saying about the foetus (Mavroforou, Koumantakis, \& Michalodimitrakis, 2010). Thus, he may believe that because the foetus is in his spouse's body, she has the final say over it (Hales, 1996). It is important to understand why some partners are tolerable with regard to prenatal alcohol use, as this may present ways to influence this tolerance. More research is subsequently needed to find out whether a decrease in tolerance with regard to prenatal alcohol use will lead to an increase in partner support. 
This study was not designed to confirm that a high amount of selfreported partner support actually relates to low prenatal alcohol use. First, it is unclear whether the self-reported partner support reflects actual support. Future research is recommended to investigate the validity of these self-reports. Moreover, it is not clear how this selfreported support relates to the pregnant spouse's perception of this support. Previous research found only a weak relation between partner support as perceived by the pregnant woman and provided by the partner. McNamara and colleagues (2006) explain this discrepancy as a representation of a disconnect between what pregnant women need and what partners think is provided in terms of support. More research is needed to replicate this finding in other samples. Finally, it is uncertain how partner support is related to actual prenatal alcohol use. A previous cross-sectional study showed that support as reported by the partner was negatively related to the alcohol use of his pregnant spouse (Van der Wulp, Hoving, \& De Vries, 2014a). However, more longitudinal research is recommended to reveal whether partner support is significant in predicting prenatal alcohol use.

\section{Strengths and limitations}

The strength of this study is that it is, to our knowledge, the first to address the correlates of partner support to abstain from prenatal alcohol use. By focusing on partner support, this study provides new insights, expanding the research area aiming to reduce prenatal alcohol use.

This study is also subject to some limitations. First, partner support was measured by one item, i.e. "I support my spouse not to drink alcoholic beverages during her pregnancy". In order to avoid a list of too many questions we opted to measure this by one question. Responses to this question may have been subject to social desirable answers. More research is needed to obtain more specific indicators of partner support, such as listing various actions that are indicators for support or combining support scores from partners with experienced 
support by their pregnant spouse. Second, midwives may have made a selection of partners to invite for participation, even though midwives were instructed to invite all partners. Online data collection might also have contributed to a participation bias. It was decided to use the Internet for data collection as the advantages (e.g. improved data input accuracy, reduced costs and increased response rates; Gosling, et al., 2004; Bishop, et al., 2010) outweighed this disadvantage and others (such as the potential of higher drop-out rates due to the less personal nature of online studies; Murray, 2007). Although a selection bias may have been present, a wide range of support levels were found in our sample, providing insight in differences between partners high versus low in partner support. Third, the study had a cross-sectional design. Longitudinal research is needed to examine the causality of the relationship between the investigated factors and partner support.

\section{Conclusion}

This is the first study on self-reported partner support to abstain from prenatal alcohol use, showing differences between partners high versus low in partner support. This study provides indications for how to use partner support to prevent prenatal alcohol use. More research is recommended to explore interventions to increase partner support, as effective interventions to increase partner support may lead to a decrease of prenatal alcohol use. 


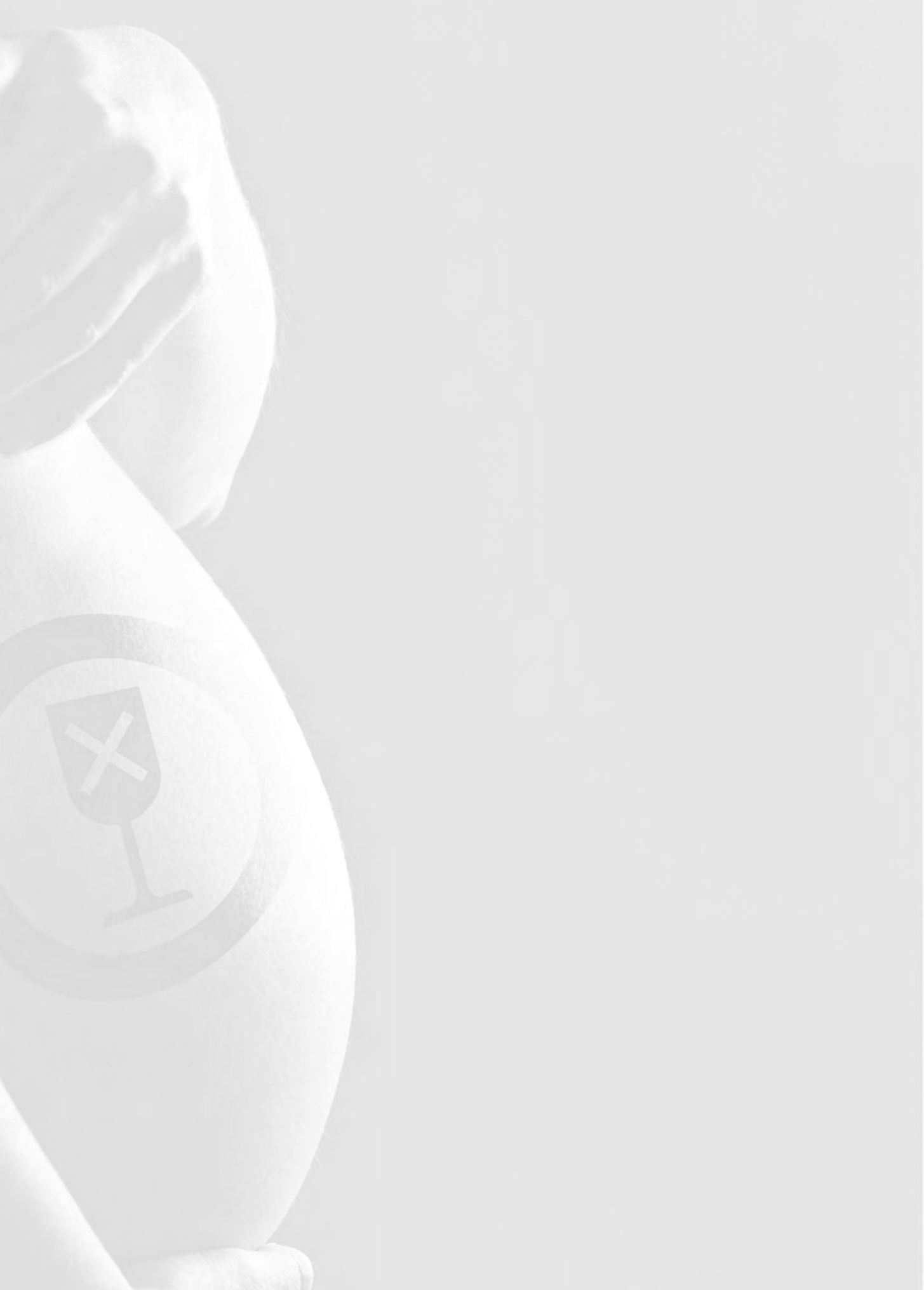




\section{CHAPTER 4}

\section{A qualitative investigation of alcohol use advice during pregnancy: Experiences of Dutch midwives, pregnant women and their partners}

Published as: Van der Wulp, N.Y., Hoving, C., de Vries, H. (2013). A qualitative investigation of alcohol use advice during pregnancy: experiences of Dutch midwives, pregnant women and their partners. Midwifery, 29(11): e89-98. 


\section{Abstract}

Objective: Two studies aimed to explore the advice Dutch midwives give and the information Dutch pregnant women and partners of pregnant women receive about alcohol consumption in pregnancy.

Methods: Study 1 included individual semi-structured interviews with 10 midwives. Study 2 involved focus groups and individual semi-structured interviews with 25 pregnant women and 9 partners. Interview content was based on the I-Change Model. Study 1 was conducted nation-wide; Study 2 was conducted in the central and southern regions of the Netherlands.

Results: Study 1 showed that midwives intended to advise complete abstinence, although this advice was mostly given when women indicated to consume alcohol. Midwives reported to lack good screening skills and sufficient knowledge about the mechanisms and consequences of prenatal alcohol use and did not involve partners in their alcohol advice. In Study 2, the views of pregnant women and partners were congruent to findings reported in Study 1. In addition, pregnant women and partners considered midwives as an important source of information on alcohol in pregnancy. Partners were interested in the subject, had a liberal view on prenatal alcohol use and felt ignored by midwives and websites. Pregnant women indicated to receive conflicting alcohol advice from their health professionals.

Conclusions: Midwives' alcohol advice requires improvement with regard to screening, knowledge about mechanisms and consequences of prenatal alcohol use and the involvement of the partners in alcohol advice during pregnancy. Training should be given to Dutch midwives to increase their screening skills and their alcohol related knowledge to pregnant women. Research is needed to determine how the midwife's alcohol advice to the partner should be framed in order to optimize the partner's involvement concerning alcohol abstinence in pregnancy. More attention to the topic at a national level, for example via mass media campaigns, should also be considered to change views about alcohol use during pregnancy in all stakeholders. 


\section{Introduction}

Maternal prenatal alcohol use is one of the leading preventable causes of mental retardation in children (Centers for Disease Control and Prevention, 2002). The range of damage includes mild and subtle changes, such as slight learning difficulties or deviant behavior, through full-blown Fetal Alcohol Syndrome (FAS) which is characterized by severe learning disabilities, growth deficiencies, abnormal facial features, and central nervous system disorders (O'Leary, 2004). Due to accumulating evidence that even low levels of prenatal alcohol exposure could cause adverse neurobehavioral effects in children (Nathanson, Jayesinghe, \& Roycroft, 2007), many Western countries, such as the United States, Australia and the Netherlands, officially recommend that pregnant women completely abstain from alcohol (American Academy of Pediatrics Committee on Substance Abuse, 1993; Health Council of the Netherlands, 2005; National Health and Medical Research Council, 2009). Nevertheless, more than $20 \%$ of pregnant women worldwide consume alcohol (Chang, et al, 2000), with estimations mounting to 35$50 \%$ in the Netherlands (Health Council of the Netherlands, 2005).

Comprehensive alcohol advice has been shown to be effective in reducing prenatal alcohol use (Chang, 2004). However, research has revealed that presently this advice is often insufficiently implemented (Jones, et al., 2011). Consequently many pregnant women remain ignorant of the consequences of prenatal alcohol use and underestimate the risks (Butters \& Howie, 1990; Floyd, Decouflé, \& Hungerford, 1999; Kesmodel \& Schioler Kesmodel, 2002; Toutain, 2010). Cognitive behavioral models such as the Health Belief Model (HBM; Janz \& Becker, 1984) suggest that advice aimed to change risky behavior should include information which illuminates the risks of the behavior and increases self-consciousness that someone engages in this risky behavior. Indeed, research by Chang (2004) confirmed that information about the health risks and other outcomes of alcohol use in pregnancy as well as feedback about pregnant women's alcohol use increasing their awareness are important elements of effective alcohol advice. 
Another effective component of advice aimed to increase alcohol abstinence in pregnancy is the inclusion of the partner of a pregnant woman. Indeed, several studies confirm that the expectant father or partner influences the behavior of a pregnant woman. One study with pregnant women in their third trimester found that social support was significantly related to the reduction of alcohol use in pregnancy (Coleman, Coleman, \& Murray, 1990). Additionally, research showed that the partner's smoking habit was one of the most powerful predictors of smoking cessation by the pregnant woman (Olsen, 1993; Waterson, Evans, \& Murray-Lyon, 1990). Furthermore, a study testing the effectiveness of a brief intervention in the reduction of prenatal alcohol consumption found enhanced effects when the brief intervention was given to both the pregnant woman and her partner instead of to only the pregnant woman (Chang, et al, 2005). This variety of research evidence is in line with some cognitive behavioral models such as Theory of Planned Behavior (TPB; Ajzen, 1985), suggesting that social influence is an important factor of behavior change.

Thus, alcohol abstinence in pregnancy is expected to increase when pregnant women are given comprehensive alcohol advice, and when this information is also addressed to the partner. Providing alcohol advice in pregnancy is often considered to be the responsibility of midwives (Kesmodel \& Kesmodel, 2011; Wiegers, Van der Velden, \& Hingstman, 2005). Research in Denmark, Sweden and Australia has revealed that midwives encounter difficulties in providing this advice (Kesmodel \& Kesmodel, 2011). For instance, midwives' advice is hindered by their perceived lack of detailed knowledge about the risks of alcohol use in pregnancy and by their perceived underdeveloped skills to deliver advice without distressing women who have already used alcohol in pregnancy (Kesmodel \& Kesmodel, 2011; Jones, et al, 2011). Moreover, research showed that midwives found it difficult to screen for pregnant women consuming alcohol (Holmqvist \& Nilsen, 2010).

The difficulties of midwives with regard to providing good alcohol advice can be related to the dissatisfaction of pregnant women with the advice they receive. Pregnant women often do not recall being actively engaged 
by the midwife in a discussion about alcohol consumption (Jones, et al, 2011) and when they receive alcohol advice, they describe this advice as being confusing, unclear or lacking sufficient detail (Jones, et al, 2011; Kesmodel \& Schioler Kesmodel, 2002; Raymond, et al, 2009).

The present research aimed to extend previous research on providing and receiving alcohol advice in two ways. First, this research focused on the Dutch situation with regard to alcohol advice in pregnancy. Due to the differences between midwifery systems (McKay, 2004; Kateman \& Herschderfer, 2005), the findings of the Danish, Swedish and Australian studies may not be applicable to the Dutch situation. Thus, at present, it is unclear whether the difficulties encountered by midwives in Denmark, Sweden and Australia are also met by midwives in the Netherlands. Moreover, it is unknown how Dutch pregnant women experience the information they receive about alcohol in pregnancy. Neither topic has yet been investigated in the Netherlands. It is important to explore the situation in the Netherlands as the Dutch midwifery system plays a pivotal role for Dutch pregnant women with $75 \%$ of the Dutch women at the beginning of their pregnancy and $36 \%$ of the Dutch women throughout their entire pregnancy receiving care only from a midwife (Wiegers, et al, 2005). Second, this research aimed to extend previous research by focusing on the experiences of partners with regard to receiving alcohol advice in pregnancy. Thus far, no research has been conducted to assess whether partners are included in midwives' alcohol advice and how partners evaluate this advice. Previous research has shown that discussing alcohol use in pregnancy is a sensitive subject (Jones, et al., 2012). When studying a sensitive subject, it is important to leave the participants' perspective in tact while collecting rich, detailed and valid data. As recommended by Steckler and colleagues (1992), a qualitative design is most suitable for this purpose.

The I-Change Model (De Vries, et al, 2003, 2008; see Figure 4.1) served as the theoretical framework of this research. This model incorporates concepts from several social cognitive models, including the HBM (Janz \& Becker, 1984) and the TPB (Ajzen, 1985). The concepts included in the I-Change Model have been constructed and tested within European 
populations. The I-Change Model has been used successfully to explain a variety of health behaviors, including alcohol use and prenatal smoking (De Vries, et al, 2006a), postnatal care (Kools, Thijs, \& De Vries, 2005a) and adoption processes (Segaar, et al, 2007). The I-Change Model assumes that individual behavior is most indirectly determined by preceding factors and information factors. Preceding factors (also referred to as predisposing factors) pertain to behavioral factors (e.g. other substance abuse), psychological factors (e.g. level of neuroticism), and socio-cultural factors (e.g. acceptance of alcohol consumption in a society); information factors pertain to factors that determine the quality of the messages (De Vries, \& Mudde, 1998), and include message factors (e.g. the utilization of positive or negative frames; Van 't Riet, et al, 2010) and the type of sources (e.g. potential differences of a GP or midwife explaining the effects of alcohol to a pregnant women). Both preceding and information factors influence the processing of information by raising awareness, creating motivation and behavior change. Readiness to process information will be dependent on a level of awareness of a problem. Factors that increase levels of awareness are knowledge, risk perceptions and cues to action (e.g. campaigns about the dangers of alcohol during pregnancy). When a person is aware of a certain health problem, such as alcohol for the unborn baby, he or she will be motivated to process information further. Motivation to change unhealthy behavior is then influenced by attitudes (perceptions of pros and cons of the desired behavior), social influence perceptions (e.g. support by their partner; De Vries et al, 1994) and self-efficacy (feeling confident to realize the behavior in difficult situations; Bandura, et al, 1988; De Vries, Dijkstra, \& Kuhlman, 1988; De Vries, et al, 1994). A high level of motivation determines a person's intention to change. Yet, a positive intention does not automatically translate into behavior. For this, again self-efficacy and action planning strategies (e.g. I will tell my friends that I will not drink alcohol anymore) and skills are important factors (De Vries, et al., 2008, Gollwitzer, 1999; Locke \& Latham, 2002; Van Osch, et al., 2010). 


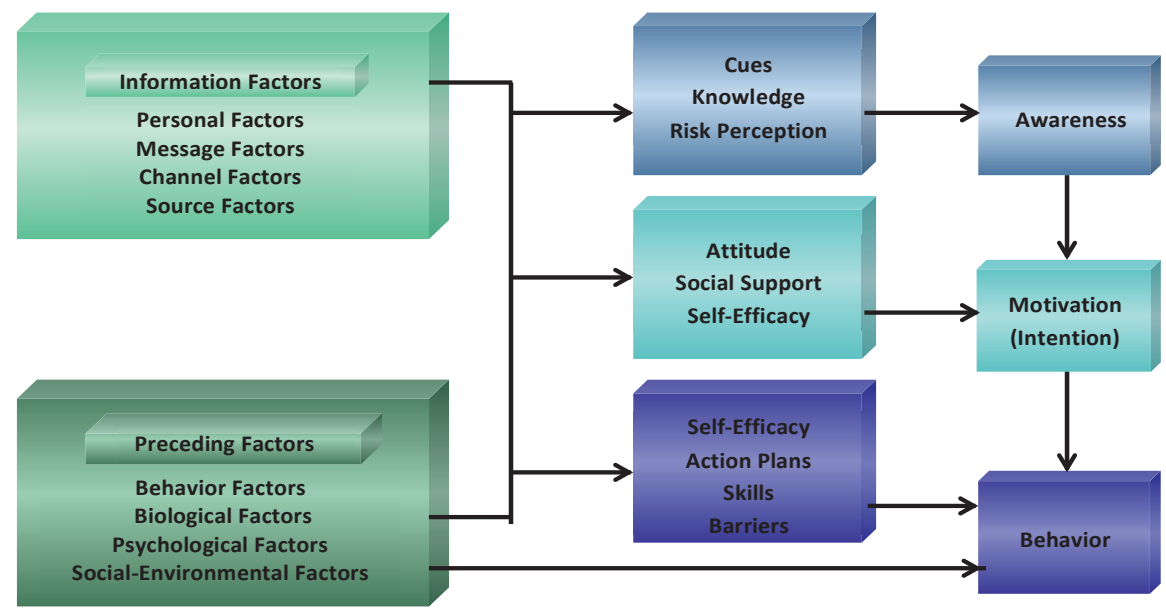

Figure 4.1 The I-Change Model 2.1

The I-Change Model was used in two studies, both presented here. The first study aimed to explore what alcohol advice Dutch midwives give to their clients. The second study aimed to explore what information Dutch pregnant women and partners receive about alcohol in pregnancy.

\section{Methods}

A qualitative design was used to optimally capture the experiences of midwives, pregnant women and partners with regard to alcohol use advice during pregnancy. In Study 1, ten interviews were conducted with midwives to gain insight into their provision of information about alcohol in pregnancy. In Study 2, five focus groups and four interviews with a convenience sample of pregnant women and their partners were conducted to gain insight into the information pregnant women and partners receive about alcohol in pregnancy. The respondents of Study 1 and 2 were recruited from different practices, assuring that the respondents of Study 1 could not influence the results of Study 2 and vice versa. Data saturation was reached before cessation of the interviews (Francis, et al, 2010). Ethics approval for this research was granted by a human research ethics committee (METC Atrium MC Maaslandziekenhuis; 08-N-50). 


\section{Study 1: Individual interviews with midwives}

\section{Procedure}

Interviews with midwives were conducted between September and December 2009. To obtain a variation in participating midwife practices, midwives were recruited on the basis of geographical location and type of midwife practice. Ten midwife practices were initially invited by email, with information about the purpose of the study. Five midwife practices were not able or willing to participate, mostly due to time constraints. Therefore, five additional midwife practices were approached; all of them consented to their participation. Two participating practices were located in each part of the Netherlands (north, east, south, west and central), one located in an urban and another in a rural setting. Interviews lasted approximately 30 minutes. Three midwives were interviewed by telephone; detailed notes were taken. Seven interviews were face-to-face; these interviews took place at the midwife's practice and were audio-taped.

\section{Measurement}

The interview routing was semi-structured in nature, allowing for midwives to raise additional subjects during the interviews. Questions were based on concepts of the I-Change Model (De Vries, et al, 2003). Table 4.1 shows the interview question route for midwives. 
Table 4.1 Midwife interview question route

Preceding factors

Principal questions: What do you think of alcohol use in pregnancy? Do you use alcohol yourself?

Discussion topics:

Do you believe many pregnant women use alcohol?

Do you think your clients use alcohol in pregnancy? Can you estimate how many?

Do you believe that your clients are honest about their alcohol use, when you ask

them about it?

Do you drink alcohol yourself?

How often do you drink alcohol?

Behavior

Principal question: What is your advice to your clients with regard to alcohol in pregnancy?

Discussion topics:

What kind of advice do you give?

Do you give the same advice to all of your clients?

During which consultation do you discuss alcohol in pregnancy?

How much time do you spend on this topic?

Do you include clients' partners in your alcohol advice? How do you include them?

Do you use tools to provide information about alcohol in pregnancy, such as

written material or websites?

Are there circumstances which make it easier or more difficult for you to discuss

alcohol with your clients?

Information factors

Principal question: Where do you obtain information about alcohol in pregnancy?

Discussion topics:

How do you keep your knowledge up to date?

How do you know that the information you receive is reliable?

Awareness factors

Principle question: What do you know about the consequences of alcohol in pregnancy? Discussion topics:

In which phase of pregnancy do you think alcohol can be dangerous for mother and unborn child?

What are the risks of alcohol use in pregnancy?

What is a safe amount of alcohol in pregnancy?

How risky is alcohol use in pregnancy?

How serious are the consequences of alcohol use in pregnancy?

Attitudes

Principle question: What are advantages and disadvantages of giving alcohol advice to your clients?

Social influence perceptions

Principle question: What do your colleagues think about alcohol in pregnancy?

Discussion topics:

Do you discuss alcohol advice with your colleagues?

Do they consider it important to give alcohol advice to pregnant women?

Which alcohol advice do they give their clients?

Which institutions or people are important for you to help you provide alcohol to your clients?

Self efficacy expectations

Principal question: Do you find it difficult to discuss alcohol with your clients?

Discussion topics:

When is it difficult for you? Can you give an example of a difficult situation?

Do you feel competent in advising your clients not to drink alcohol in pregnancy?

Why or why not?

Would you like to obtain certain skills to give alcohol advice?

Do you feel competent to determine whether and how much alcohol your clients

drinks?

Do you believe you lack knowledge about alcohol in pregnancy? 
Table 4.1 (continued). Midwife interview question route

Intention

Principal question: Do you have the intention to provide a different advice about alcohol in pregnancy in the future?

Discussion topics:

Why or why not?

Action planning

Principal question: What could help you to give good advice on alcohol in pregnancy?

Discussion topics:

Do you think your advice on alcohol consumption during pregnancy can be improved? How?

\section{Study 2: Focus groups and individual interviews with pregnant women and partners}

\section{Procedure}

From April to June 2011, five focus groups were conducted. Focus group 1 consisted of five pregnant women not using alcohol during their pregnancy. Focus groups 2 and 3 consisted of six pregnant women with respectively one and two pregnant women consuming alcohol. Focus group 4 was conducted among five partners of pregnant women (two partners of pregnant women consuming alcohol). Focus group 5 consisted of four couples (one pregnant woman consuming alcohol). Additionally, four individual interviews were conducted with pregnant women consuming alcohol.

Participants were recruited through midwife practices, pregnancy courses, prenatal childbearing classes, and pregnancy yoga classes. Midwives practicing in the central and southern regions of the Netherlands were invited to participate in this study by email and telephone. Midwives willing to contribute to the study received recruitment flyers that were to be distributed among their clients. Pregnant women and partners willing to participate could contact the researcher through the contact details noted in the flyers. Women and partners participating in pregnancy courses or classes were recruited at class meetings. 
Focus groups lasted about 75 minutes and took place at the research institute or at the midwife practice or pregnancy course where participants were recruited. Individual interviews lasted about 60 minutes and were conducted at the homes of the participants or at the institute. Before each session, participants were asked to give written informed consent. Focus groups were video-recorded and individual interviews were audio-taped. All participants received a $€ 15$ gift certificate.

\section{Measurement}

When pregnant women and partners were interested to participate, they received a screening questionnaire, consisting of seven questions regarding gender, age, place of residence, level of education (low, medium or high educational level), numbers of weeks pregnant, numbers of pregnancies experienced (current pregnancy included) and prenatal alcohol use (yes/no). Prenatal alcohol use determined the allocation of participants to a type of focus group or interview.

The interview routing was semi-structured in nature, allowing participants to raise additional subjects during the interviews. Questions were based on concepts of the I-Change Model (De Vries, et al, 2003). During the interview, the pregnant woman's alcohol consumption (i.e. behavior), whether partners discussed alcohol in pregnancy, information factors and awareness factors were explored. Table 4.2 shows the interview question route for the (focus group) interviews with pregnant women and partners. 
Table 4.2 Interview question route for pregnant women and partners

\section{Behavior}

Principal question for pregnant women not using alcohol: Is it a conscious decision not to use alcohol during pregnancy? Can you tell more?

Principal question for pregnant women using alcohol: Can you tell more about using alcohol during your pregnancy?

Principal question for partners: Has your partner used alcohol in her pregnancy?

Partners' discussion about alcohol in pregnancy

Principal question: Have you discussed prenatal alcohol use with your partner?

Discussion topics:

$$
\begin{aligned}
& \text { What did you talk about specifically? } \\
& \text { What does your partner think of alcohol use in pregnancy? }
\end{aligned}
$$

Information factors

Principal question: Did you receive or search for information about alcohol consumption during pregnancy?

Discussion topics: Source of information

From whom did you receive information about alcohol in pregnancy?

Did you search for this information yourself? Where?

When did you receive information about alcohol in pregnancy?

How did you know that the information you received about alcohol in pregnancy

was true and reliable?

In your opinion, which sources give reliable information about alcohol in pregnancy?

Have you ever received conflicting information?

From whom or what would you like to receive information about alcohol in pregnancy?

How would you have liked to receive information about alcohol in pregnancy?

When would you have liked to receive information about alcohol in pregnancy?

How do you feel about receiving information about alcohol advice in pregnancy

from your midwife?

Discussion topics: Content of information

How do you feel about the information you received about alcohol in pregnancy?

In your opinion, how can information about prenatal alcohol use be improved?

What type of information would make you reflect on the issue of alcohol in pregnancy?

Awareness factors

Principle question: What do you know about the use of alcohol in pregnancy?

Discussion topic:

How do you think that alcohol use in pregnancy affects your baby?

\section{A preliminary version of the interview guide was tested by research experts on question route design and content, as well as a pregnant woman and her partner; the interview guide was adjusted according to their comments.}




\section{Analysis of the data of midwives, pregnant women and partners}

Videotapes and audiotapes were transcribed in abbreviated form. A qualitative content analysis method was used to analyze the interviews. This involved identifying, coding and categorizing the primary patterns that emerged from the collected data (Krippendorff, 1980; Patton, 1990). For this purpose, transcripts were read through repeatedly to obtain a composite perspective. Sentences or phrases with information relevant to the questions were selected and merged into main categories, based on concepts of the I-Change Model.

\section{Findings}

\section{Study 1: Midwives}

\section{Sample}

A total of 10 midwives were interviewed. The age of the midwives ranged from 27 to 49 years. On average, they had 9 years of professional experience. A more detailed description of the sample is presented in Table 4.3. As there were no clear differences in responses between subgroups of midwives with regard to urban/rural setting, type of practice or having followed an additional course on prenatal alcohol use, the results of the total sample are presented.

\section{Behavior}

Eight of the ten midwives declared that they recommended complete abstinence to all of their clients, which meant that they advised all of their clients not to drink any alcohol, including on special occasions.

"... During the first visit I always ask the client: "do you smoke cigarettes, do you drink alcohol, do you use drugs?”. Generally, clients respond that they do not use alcohol. Sporadically, a client mentions that a glass once in a while is not harmful. I always advise my clients not to drink any alcohol." (Midwife 2) 
Table 4.3 Characteristics of the sample of midwives

\begin{tabular}{ll}
\hline Characteristic & Midwives $(\mathrm{N}=10)$ \\
\hline Female (N) & 9 \\
Mean age (SD) & $34.4(7.6)$ \\
Mean years of profession (SD) & $8.9(4.7)$ \\
Received information on prenatal alcohol use during midwife's & 9 \\
training (N) & 3 \\
Followed additional course on prenatal alcohol use (N) & \\
Place of residence (N) & 5 \\
$\quad$ City & 5 \\
$\quad$ Village/Rural & \\
Type of midwife practice (N) & 1 \\
Solo & 3 \\
Duo & 6 \\
Group & 8 \\
Recommends prenatal abstinence (N) & 5 \\
Uses alcohol (N) & 5 \\
\hline
\end{tabular}

One midwife did not give any advice, considering it unnecessary as she was convinced that her clients already abstained from alcohol. One midwife advised her clients not to drink alcohol except on special occasions as she considered light prenatal alcohol use to be harmless.

“... When a client asks me if she can drink a glass of alcoholic beverage at a special occasion, for example when she is getting married, than I tell her that is ok." (Midwife 3)

Midwives asked the pregnant woman whether she used alcohol as part of the regular substance use assessment (i.e. tobacco, alcohol and drug use) during the first visit. Most clients responded negatively. Followup questions about this denial were asked by two midwives, which resulted in a minority of the clients admitting their alcohol use. A minority of midwives also asked whether the woman's partner used alcohol. The majority of the midwives only gave alcohol advice to clients admitting their alcohol use. This was despite the fact that half of the midwives doubted the truthfulness of their clients about their alcohol consumption. 
"... I wonder if my clients are honest. In my whole career, there was only one client who admitted that she found it difficult not to drink alcohol. I do not believe she was the only one in over ten years..." (Midwife 8)

Besides verbal advice, the majority of midwives provided their clients with generic written prenatal information, in which a small section related to alcohol use. Furthermore, a few midwives referred clients to a Dutch website specifically focused on alcohol use during pregnancy (STAP, 2012).

\section{Information factors}

The midwives had learned most about alcohol in pregnancy during their midwifery training. In addition, a minority of the midwives had followed voluntary extra courses on alcohol in pregnancy organized by the midwifery association (Royal Dutch Organization of Midwifery; KNOV). Finally, midwives obtained information about alcohol in pregnancy from the media, such as television programs and interviews.

"... I heard last year that it is ok to drink one glass of alcohol when you are pregnant. I hope a gynecologist does not say that on television when it is not true." (Midwife 10)

All of the midwives declared that information obtained during their midwifery training and from the midwifery association was reliable.

"... I judge the reliability of information by the source. I regard information from the midwifery association as trustworthy." (Midwife 2)

\section{Awareness factors}

Only a few midwives knew the present official recommendations of no safe amount of prenatal alcohol use. Moreover, many midwives stated that they considered small amounts of alcohol to be harmless. 
"... When you drink two glasses of wine at Christmas or on New Year's Eve, well, that really will not do any harm" (Midwife 7)

Midwives reported several misconceptions regarding the mechanisms and consequences of alcohol in pregnancy. For example, although all midwives knew that alcohol can reach the fetus, the majority wrongly believed that alcohol could not reach the embryo as long as the development of the maternal blood supply to the placenta is incomplete.

“... Women often feel guilty when they drink alcohol before they knew they were pregnant. I try to downgrade their feelings of guilt by telling them that alcohol is not dangerous when there is no blood contact between mother and child" (Midwife 3)

Most midwives knew of Fetal Alcohol Syndrome (FAS) as a consequence of prenatal alcohol use, although none of them could correctly define it.

"... I know that facial abnormalities and growth deficiencies characterize FAS, but I don't know if there are additional characteristics" (Midwife 5)

\section{Attitude}

Most midwives considered the greatest advantage of recommending complete abstinence of prenatal alcohol use that this contributed to the health of the baby. The greatest disadvantage of providing this advice during prenatal care sessions was that a pregnant woman could develop feelings of guilt or anxiety when unwittingly having consumed alcohol while pregnant.

"... A woman, who had been drinking before receiving this advice, may feel guilty. I nevertheless find it important to advise complete abstinence. The greatest advantage is the benefits for the health of the baby." (Midwife 4) 


\section{Social influence perceptions}

Most midwives reported that discussions with colleagues could lead to adjustments in the alcohol advice for their clients. Midwives assumed that they gave the same advice about alcohol in pregnancy as their colleagues. However, some had never talked with their colleagues about this topic. Half of the midwives mentioned the midwifery organization as an important source of information regarding prenatal alcohol advice.

"... If the midwifery association publishes research which supports a certain way of providing alcohol advice, I will follow that." (Midwife 1)

\section{Self-efficacy expectations}

In general, midwives reported that they felt confident in advising their clients to abstain from alcohol in pregnancy. For a minority of midwives a lack of knowledge was a reason to discuss alcohol only shortly. Other difficulties mentioned were comforting clients who had already used alcohol in pregnancy or obtaining truthful information about the clients' alcohol use.

“... I don't find it difficult to discuss alcohol use with my clients. It is only difficult when I make my clients anxious when they had alcohol at an earlier stage." (Midwife 2)

\section{Intention}

Midwives intended to retain the content and the procedure of giving alcohol advice. Yet, reasons to change their alcohol advice were being urged by colleagues or the midwifery association or new scientific insights.

"... When research shows that prenatal alcohol use is not harmful, then I would change my recommendations. But I do not believe that will happen. So, no, I will not change my advice in the future." (Midwife 4) 


\section{Action planning}

Most midwives believed that their alcohol advice could not be improved and thus were not planning additional actions. A minority of midwives mentioned that extra knowledge about the mechanisms and consequences of alcohol in pregnancy could make their advice better, however they did not report any plans to look for additional information.

"...I would like to have a better explanation for why women should not drink alcohol in pregnancy." (Midwife 6).

\section{Study 2: Pregnant women and partners}

\section{Sample}

A total of 34 participants (25 pregnant women and nine male partners) were interviewed. The age of the participants varied from 24 to 38 years. The majority of the sample reported a relatively high educational level. A more detailed description of the sample is presented in Table 4.4.

Table 4.4 Characteristics of the sample of pregnant women and partners

\begin{tabular}{lll}
\hline Characteristic & $\begin{array}{l}\text { Pregnant women } \\
(\mathrm{N}=25)\end{array}$ & $\begin{array}{l}\text { Partners } \\
(\mathrm{N}=9)\end{array}$ \\
\hline $\begin{array}{l}\text { Mean Age (SD) } \\
\text { Place of residence }\end{array}$ & $31.0(2.7)$ & $34.0(5.0)$ \\
$\quad$ & $80 \%$ & $56 \%$ \\
$\quad$ City & $20 \%$ & $44 \%$ \\
$\quad$ Village/Rural & & \\
Level of education & $28 \%$ & $22 \%$ \\
$\quad$ Secondary vocational school/high school degree & $72 \%$ & $78 \%$ \\
$\quad$ Higher vocational school/college degree/university & & $30.4(5.4)$ \\
degree & $29.5(4.0)$ & $78 \%$ \\
Mean number of weeks pregnant (SD) & $72 \%$ & $33 \%$ \\
Percentage first pregnancy & $32 \%$ & \\
Prenatal alcohol use & &
\end{tabular}

\section{Behavior}

Prenatal alcohol use was reported by approximately one third of the pregnant women and partners and varied from a few sips to a number of glasses during the whole pregnancy. All pregnant women who had consumed alcohol since they knew they were pregnant reported to have reduced their prenatal alcohol use since. 
"... Occasionally, I have a sip of wine. In my whole pregnancy, it totaled to less than two glasses of wine. I like to drink wine very much and I really miss it during my pregnancy, but I consciously decided to restrict my wine consumption. (Pregnant woman 7 - consuming alcohol)

\section{Partners' discussion about alcohol in pregnancy}

Non alcohol consuming pregnant women rarely discussed alcohol with their partner, whereas pregnant women consuming alcohol often indicated to do so. Both pregnant women and partners reported that these discussions included conversations about the guidelines of prenatal drinking, checking each other's view regarding prenatal drinking, and discussing decreasing or ceasing alcohol intake during the pregnancy.

“... I have checked my partner's opinion about drinking the occasional sip, because it is also his child" (Pregnant woman 24 - consuming alcohol)

Partners were more liberal than their spouses with regard to prenatal alcohol use. All of the partners of the pregnant women using alcohol agreed with her alcohol use. In addition, some of the partners of the pregnant women not using alcohol would also not mind her to drink a sip occasionally.

"... I don't believe that a sip once in a while, for example with a toast or on Christmas Eve, is harmful. So that would be fine to me." (Partner 6 - non alcohol consuming pregnant woman)

Partners often asked their pregnant spouse whether they should cut down their own alcohol use, which most of the women did not find necessary.

“... When I would like to have an alcoholic drink, my spouse says: Enjoy your drink! And then she does not even look at my glass." (Partner 1 - alcohol consuming pregnant woman) 
Nevertheless, many partners still reduced their alcohol use around their spouse to support her to refrain from alcohol and because they missed their spouse as drinking companion.

“... I do not open a bottle of wine as often as I did before my spouse was pregnant, because now I have to finish it by my self or it gets bad and I have to throw it away. Hence, I drink less because I miss my drinking companion." (Partner 1 - alcohol consuming pregnant woman)

\section{Information factors}

Pregnant women and partners obtained information about alcohol in pregnancy mostly through their midwife, general practitioner (GP) or the internet. The majority of pregnant women reported that their midwife had asked them whether they used alcohol in pregnancy, which was negated by all of the women. Some pregnant women mentioned that their midwife had advised them not to drink alcohol; however most of the pregnant women consuming alcohol and partners reported that their midwives had told them that limited alcohol use would not be harmful.

"... During the first consultation, my midwife told me that a couple of sips was no problem." (Pregnant woman 13 consuming alcohol)

Compared to non alcohol consuming pregnant women, women consuming alcohol more often reported having received conflicting information from different sources about the harmfulness of alcohol.

"... The midwife told me it is better not to drink alcohol at all. However, I once visited my GP and we discussed alcohol use in pregnancy. He said: 'you can enjoy a glass of wine every now and then'." (Pregnant woman 24 - consuming alcohol)

In general, non alcohol consuming pregnant women were satisfied with the information they received from the midwives. In contrast, 
the majority of pregnant women consuming alcohol and partners were less satisfied; they wished that their midwives were more effective in screening for prenatal alcohol use. Moreover, they preferred having received more information on the consequences of alcohol use for the fetus, on safe amounts of alcohol in pregnancy and the mechanisms of harm due to prenatal alcohol use.

“... The midwife's alcohol advice can be improved. She can give an indication of the risks. What happens when you use alcohol. How risky is it when you drink one glass or when you drink five glasses. She can give more elaborate information instead of just stating that alcohol is not good." (Pregnant woman 25 consuming alcohol)

Respondents only considered websites reliable when they were provided by health professionals, governments or specialized institutes.

"... The most reliable website is provided by midwife practices. Midwives make sure that the information on their website is accurate." (Pregnant woman 17 - not consuming alcohol)

Partners declared that websites about pregnancy seemed largely designed for women, as they were published in pink and contained pictures of happy pregnant women. These partners proposed that a different design of websites or a section especially designed for expectant fathers would increase the involvement of partners with regard to prenatal alcohol use.

"As a partner, your presence often feels pointless ... If you want to provide information to men about alcohol in pregnancy, it would help if men feel that the information is addressed specifically to them." (Partner 4 - non alcohol consuming pregnant woman)

\section{Awareness factors}

Pregnant women not using alcohol were able to report in more detail on the consequences of prenatal alcohol use compared to women using 
alcohol. Fetal Alcohol Syndrome as a possible consequence of prenatal alcohol use was mentioned only by a few participants, who were all pregnant women not using alcohol or partners. Compared to pregnant women not using alcohol, women using alcohol and partners more often believed that small amounts of alcohol were not harmful.

"... We all know that excessive and prolonged alcohol consumption is very risky for the baby. So, we would never do that. But there is no evidence that light drinking is harmful." (Partner 3 - alcohol consuming pregnant woman)

\section{Discussion}

Study 1 revealed that the majority of the interviewed Dutch midwives recommended complete abstinence. However, they did not provide pro-active but only reactive advice, when clients admitted alcohol consumption. Given the fact that alcohol consumption is likely to be underreported (Morrow-Tlucak, et al, 1989), this implies that Dutch midwives may not provide information about the dangers of alcohol even though women consume alcohol. A recommendation to overcome this issue is to introduce a standard advice about alcohol consumption for any pregnant woman.

Moreover, the findings of Study 1 suggest that the Dutch midwives' advice is suboptimal as most of the interviewed midwives failed in screening for prenatal alcohol use and their information about the mechanisms and consequences of alcohol in pregnancy was too limited. These difficulties have also been reported by midwives in previous studies (Kesmodel \& Kesmodel, 2011; Holmqvist \& Nilsen, 2010; Jones, et al, 2011). A Swedish national education project showed that the use of the Alcohol Use Disorders Identification Test (AUDIT) in the regular consultations of prenatal care improved the screening of prenatal alcohol use (Skagerström, et al, 2012). Moreover, the knowledge of midwives with regard to alcohol in pregnancy was improved by the project's facilitation of training in general information on alcohol- 
related health issues (Skagerström, et al, 2012). The role of the Swedish midwives in educating pregnant women is comparable to the Dutch situation (Kateman \& Herschderfer, 2005). It is therefore recommended that the possibilities are explored of adopting effective components of the Swedish project to improve the alcohol advice of Dutch midwives.

Study 1 also demonstrated that the interviewed midwives largely ignored their clients' partner in their alcohol advice. Previous research has demonstrated the effect of the partner on a pregnant woman's behavior (De Vries, et al, 2006a; Ginsberg, Hall, \& Rosinski, 1991; Koshy, et al, 2010; McBride, et al, 2004; Tarrant, et al, 2010; Wolfberg, et al, 2004). Accordingly, by not involving the partner in her alcohol advice, the midwife may miss an opportunity to increase alcohol abstinence. More research is needed to find out how the midwife's alcohol advice to the partner should be framed to optimize the partner's influence on increasing alcohol abstinence in pregnancy.

The findings of Study 1 were confirmed in Study 2, which additionally demonstrated that pregnant women and partners considered the midwife as an important and reliable source of information on alcohol in pregnancy. This suggests that there may be a relation between the midwives' limited knowledge concerning alcohol and pregnancy and the low level of knowledge in pregnant women and partners reported in Study 2. However, the present study was not designed to establish this relation and we are not aware of any other research supporting this. Nevertheless, there is evidence that the pregnant women's knowledge is weakly negatively associated with prenatal alcohol consumption (Chang, et al, 2006a). Thus, the low level of knowledge in pregnant women found in the present study, which can be considered as a precondition for further processing information influencing attitude and self-efficacy beliefs, clearly suggests that clear messages for this target group about the effects and dangers of alcohol for their unborn child is required.

With regard to the partners' opinion about the alcohol advice in pregnancy, Study 2 showed three main findings: the interviewed partners were dissatisfied with the limited amount of information provided by 
midwives and their poor screening skills; partners' view on alcohol in pregnancy was more liberal than their pregnant spouse's; and partners were actively involved in the subject of alcohol use in pregnancy, as they discussed this issue with their spouses and appreciated an objective website about alcohol in pregnancy. Because this is to our knowledge the first study investigating partners' experiences with alcohol advice in pregnancy, we cannot generalize the findings to other situations. However, the previously demonstrated effect of the partner on a pregnant woman's behavior (De Vries, et al, 2006a; Ginsberg, et al, 1991; Koshy, et al, 2010; McBride, et al, 2004; Tarrant, et al, 2010; Wolfberg, et al, 2004), implies that it is worthwhile to improve the midwives' advice and encourage her to direct it towards the partner as well. This is expected to increase partner's level of satisfaction concerning this advice as well as a more positive view on complete prenatal alcohol abstinence. As the partner is actively involved in the subject, he may have an influence on the reduction of his pregnant spouse's alcohol use.

Finally, Study 2 showed that especially pregnant women consuming alcohol had received conflicting alcohol advice from their health professionals. Apparently, not all Dutch health professionals are convinced that complete abstinence yields to better pregnancy outcome. Research has shown that inconsistent or conflicting advice by health professionals undermines women's confidence and efforts to breastfeed (Hailes \& Wellard, 2000; Hauck, et al, 2011). The national Health Department as well as professional associations are recommended to make an effort to ensure that all health professionals provide the same alcohol advice in pregnancy.

\section{Strengths and limitations}

This study has several strengths. This is the first study investigating how partners perceive alcohol advice in pregnancy, along with pregnant women and midwives. By also focusing on the partner, this study provides new insights, expanding the research area aiming to reduce prenatal alcohol use. Moreover, this study is based on a 
theoretical framework, which has been used in a variety of research on a diversity of health behaviors (e.g. De Vries, et al, 2006b; De Vries, et al, 2003; Hoving, Reubsaet, \& De Vries, 2007b). Finally, the qualitative character of this study allowed the exploration of the Dutch situation of midwives, pregnant women and partners with regard to alcohol advice in pregnancy. In order to generalize to a larger population and to assess determinants of providing or accepting alcohol advice in pregnancy, it is recommended that subsequent research quantifies the findings of the present research (Steckler, et al., 1992).

This study is also subject to some limitations. First, the sample size was relatively small. However, data saturation was reached before cessation of the interviews. Second, responses given may have been subject to a social desirability bias. However, strategies to reduce the social desirability bias were applied, such as wording questions about channels of information in a neutral fashion as well as creating an atmosphere of acceptance through nonjudgmental listening and showing consideration for the answers given by the respondents (Langeland, Drayer, \& Van den Brink, 2003). Future research may consider an alternative data collection method which is less prone to the social desirability bias. For example, camera recordings can be used during booking visits to measure when and what kind of information midwives provide and how it is received by pregnant women and their partners. Di Matteo and colleagues (2003) provided evidence for the validity of camera recordings of physicianpatient visits compared to medical chart records provided by the physician. On the downside, the use of camera recordings is more prone to refusals to participate (Coleman, 1996) and may increase selfawareness, which subsequently enhances the social desirability bias. An alternative method is computer-assisted, self-administered interviews (CASI), which has been demonstrated to produce more valid reports of sensitive behaviors compared to face to face interviews (Kissinger, et al, 1999; Tideman, et al, 2007). Disadvantageously, this is a quantitative method, whereas a qualitative method is preferred for the exploration of a complex subject such as prenatal alcohol use (Steckler, et al, 1992). Finally, in the second study, the only demographical factors assessed were age and education. This showed that participants had a high 
level of education, indicating that selection bias may have occurred. Generalizability is not a main issue in qualitative studies, where it is more important to have a variation in respondents to increase understanding of complex human issues (Marshall, 1996). The presence of respondents with a lower level of education in Study 2 contributed to this variation. Ethnicity and income have not been assessed, due to restrictions of the length of the screening questionnaire. We suggest that future quantitative research also assesses these demographical background factors in order to examine a possible relation between ethnicity and socio-economical status on the one hand and giving and receiving information on a sensitive topic as alcohol consumption on the other hand.

\section{Conclusions and implications}

From the viewpoint of the midwives as well as of pregnant women and partners it can be concluded that the alcohol advice provided by midwives requires improvement with regard to screening, information about mechanisms and consequences of prenatal alcohol use and involvement of the partners. It is therefore recommended that midwives use a standard advice about alcohol consumption for any pregnant woman as well as a validated screening tool. Moreover, midwives should be offered training to increase their knowledge on prenatal alcohol use. The possibilities to implement effective components of the Swedish national education project in the Dutch prenatal care should be explored. In addition, more research is needed to find out how the midwife's alcohol advice to the partner should be framed to optimize the partner's influence on increasing alcohol abstinence in pregnancy. Finally, views about alcohol use in pregnancy in all stakeholders can most effectively be changed when information about this topic is not only directed to the individual but also to the individual's social environment. Thus, more attention for this topic at a national level, for example via mass media campaigns, is recommended. 



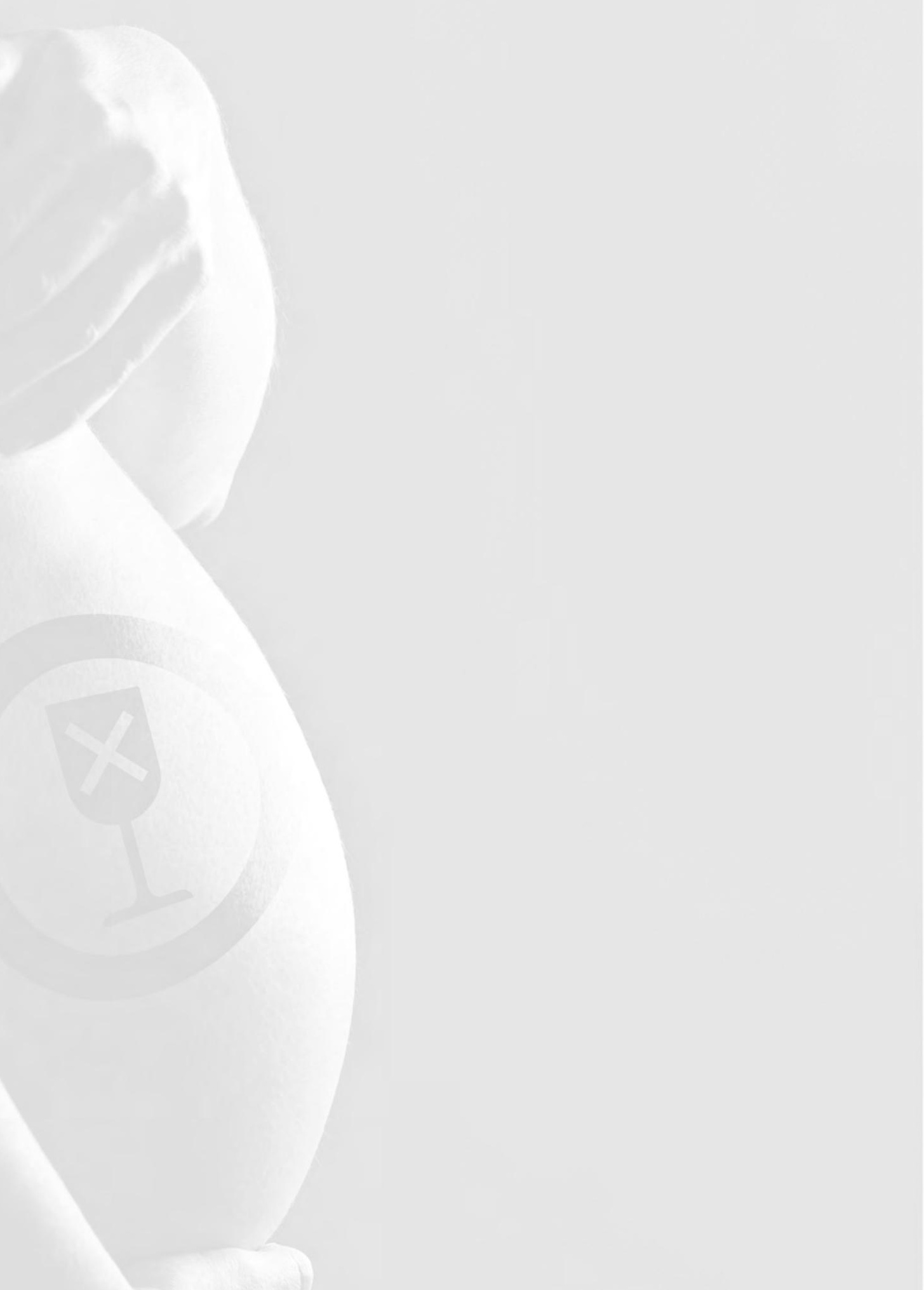




\section{CHAPTER 5}

\section{Reducing alcohol use during pregnancy via health counselling by midwives and internet-based computer-tailored feedback: A cluster randomized trial}

Published as: Van der Wulp, N.Y., Hoving, C., Eijmael, K., Candel, M.J.J.M., van Dalen, W., \& de Vries, H. (2014). Reducing alcohol use during pregnancy via health counselling by midwives and internet-

based computer-tailored feedback: A cluster randomized trial. Journal of Medical Internet Research, 16(12): e274. 


\section{Abstract}

Background:Effectiveinterventions are needed to reduce neurobehavioral impairments in children due to maternal alcohol use during pregnancy. Currently, health-counselling interventions have shown inconsistent results to reduce prenatal alcohol use. Thus, more research using health counselling is needed to gain more knowledge about the effectiveness of this type of intervention on reducing alcohol use during pregnancy. An alternative and promising strategy is computer tailoring. However, to date, no study has shown the effectiveness of this intervention mode. Objective: The aim was to test the effectiveness of health counselling and computer tailoring on stopping and reducing maternal alcohol use during pregnancy in a Dutch sample of pregnant women using alcohol.

Methods: A total of 60 Dutch midwifery practices, randomly assigned to 1 of 3 conditions, recruited 135 health counselling, 116 computer tailoring, and 142 usual care respondents from February to September 2011. Health-counselling respondents received counselling from their midwife according to a health-counselling protocol, which consisted of 7 steps addressed in 3 feedback sessions. Computer-tailoring respondents received usual care from their midwife and 3 computer-tailored feedback letters via the Internet. Usual care respondents received routine alcohol care from their midwife. After 3 and 6 months, we assessed the effect of the interventions on alcohol use.

Results: Multilevel multiple logistic regression analyses showed that computer-tailoring respondents stopped using alcohol more often compared to usual care respondents 6 months after baseline $(53 / 68$, $78 \%$ vs 51/93, 55\%; $\mathrm{P}=.04)$. Multilevel multiple linear regression analyses showed that computer-tailoring respondents (mean 0.35, SD 0.31 units per week) with average $(\mathrm{P}=.007)$ or lower $(\mathrm{P}<.001)$ alcohol use before pregnancy or with average $(\mathrm{P}=.03)$ or lower $(\mathrm{P}=.002)$ social support more strongly reduced their alcohol use 6 months after baseline compared to usual care respondents (mean 0.48 , SD 0.54 units per week). Six months after baseline, $72 \%(62 / 86)$ of the health-counselling respondents had 
stopped using alcohol. This $17 \%$ difference with the usual care group was not significant.

Conclusions: This is the first study showing that computer tailoring can be effective to reduce alcohol use during pregnancy; health counselling did not effectively reduce alcohol use. Future researchers are recommended to invest more in recruitment of pregnant women and implementation by health care providers. Because pregnant women are reluctant to disclose their alcohol use to health professionals and computer tailoring preserves a person's anonymity, this effective computer-tailoring intervention is recommended as an attractive intervention for pregnant women using alcohol. 


\section{Introduction}

Alcohol use in pregnancy is a leading preventable cause of intellectual disability in children (Centers for Disease Control and Prevention, 2002). Due to accumulating evidence that even low levels of prenatal alcohol exposure can cause adverse neurobehavioral effects in children (Nathanson, Jayesinghe, \& Roycroft, 2007), many Western countries, such as the United States, Australia, and the Netherlands, officially recommend that pregnant women completely abstain from alcohol (American Academy of Pediatrics Committee on Substance Abuse, 1993; Health Council of the Netherlands, 2005; National Health and Medical Research Council, 2009). Nevertheless, more than $20 \%$ of pregnant women worldwide consume alcohol (Chang, et al., 2000), with estimations accumulating to $35 \%$ to $50 \%$ in the Netherlands (Health Council of the Netherlands, 2005). Effective interventions are needed to reduce the number of pregnant women who endanger the health of their foetuses by using alcohol in pregnancy.

Various interventions to reduce prenatal alcohol use have been described in reviews (Gebara, et al., 2013; Nilsen, 2009; Stade, et al., 2009); however, only 5 studies used a randomized controlled trial to test intervention effectiveness (i.e., Chang, et al., 1999; Chang, et al., 2006b; Handmaker, Miller, \& Manicke, 1999; O'Connor \& Whaley, 2007; Reynolds, et al., 1995). All these interventions applied health counselling; pregnant women were screened for alcohol use and participated in motivational interviews conducted by health professionals (e.g., Miller \& Rollnick, 1991). These studies suggest that health counselling interventions may result in increased abstinence and a reduction in prenatal alcohol consumption. However, because of the inconsistency of the results, the paucity of studies, the relatively low number of total respondents, the high risk of bias of the studies due to lack of information on allocation concealment, and the complexities of interventions, many uncertainties remain about the most optimal conditions of these interventions (Stade, et al., 2009). 
An alternative and promising strategy is computer tailoring, an intervention in which advice is not delivered face-to-face, but via a computer (De Vries \& Brug, 1999). The content of this advice is based on the answers of respondents to questions and generated by a computer program. Consequently, the feedback is adapted to the specific characteristics of a particular individual, yielding the potential to provide messages highly tailored to the individuals' situation (Hawkins, et al., 2008). Computer-tailored messages have been shown to attract and keep an individual's attention (De Vries \& Brug, 1999; Kreuter, et al., 2000) more than generic advice, resulting in a more thorough processing of information (Dijkstra, 2005). Computer tailoring has proven to be effective in changing different health-related behaviours (Krebs, Prochaska, \& Rossi, 2010; De Vries, et al., 2008), such as smoking (E.S. Smit, de Vries, \& Hoving, 2012), vegetable and fruit intake (Oenema, Tan, \& Brug, 2005), and alcohol use (Boon, et al., 2011; Schulz, et al., 2013). To our knowledge, only one computer-tailored intervention for alcohol use in pregnancy has been developed. Tzilos and colleagues (2011) found that users liked the program and appreciated the ease of use. Nevertheless, they did not find any significant difference in the reduction of alcohol use compared to an assessment-only condition, perhaps because their 1-month follow-up was simply too soon to find beneficial effects of the computer tailoring or because their phone-based follow-up led to a social desirability bias concealing real decreases in drinking. Thus, it has not been shown that computer tailoring can be effective in reducing prenatal alcohol use.

The goal of this study was to test the effectiveness of two different brief interventions to reduce prenatal alcohol use: a health counselling and a computer-tailored intervention, in comparison with usual care. In agreement with several national recommendations (American Academy of Pediatrics Committee on Substance Abuse, 1993; Health Council of the Netherlands, 2005; National Health and Medical Research Council, 2009), our primary focus for the development of the interventions was that pregnant women who used alcohol in the beginning of their pregnancy stopped their alcohol use after having received an intervention. Thus, our first hypothesis was that women receiving health counselling or 
computer tailoring were more likely to stop using alcohol in pregnancy compared to women receiving usual care. However, for the pregnant women unwilling or unable to completely stop their alcohol use, we aimed at reducing their alcohol use because research has shown that the risk and severity of the effects of prenatal alcohol use are doserelated (Driscoll, Streissguth, \& Riley, 1990). Consequently, our second hypothesis is that when women continued their alcohol use, those receiving health counselling or computer tailoring were more likely to reduce their alcohol use compared to those receiving usual care.

\section{Methods}

The study was approved by the Medical Ethics Committee of Maastricht University and the University Hospital Maastricht (MEC 09-3-070) and is registered with the Dutch Trial Register (NTR2058).

\section{Sample}

A sample size analysis, with power 0.80 , alpha $=.05$, intraclass correlation coefficient (ICC) of 0.01 (reported in a previous study as the median ICC for cluster-based studies in primary care; Adams, et al., 2004), an estimated quit rate of $40 \%$ in each of the experimental conditions versus $20 \%$ in the control condition, and the estimated inclusion of 30 midwifery practices, revealed that 9 respondents per practice were needed. Estimating 10\% attrition over the trial period, we aimed to include 300 respondents at baseline. The estimated quit rate and attrition were based on a previous Dutch study on smoking cessation during pregnancy (De Vries, et al., 2006a).

Eligibility criteria were ability to understand Dutch, aged 18 years or older, pregnant for a maximum of 12 weeks (because respondents received follow-up questionnaires until 6 months after baseline), and having drunk alcohol since knowing to be pregnant. 


\section{Procedures}

Respondents were recruited from February to September 2011. Recruitment letters were sent to all midwifery practices in the Netherlands $(\mathrm{N}=540)$. Participating practices were randomly assigned to 1 of the 3 conditions (health counselling, computer tailoring, or usual care) by a computer software randomization device to avoid contamination. The practices informed their clients about the study by email or phone. When pregnant women agreed to participate, they were asked to visit the study website before their initial consultation. They could do this where and whenever they had access to the Internet.

During the recruitment period, it appeared that the inclusion of 9 respondents per practice would be too time-consuming. We decided to enrol 60 midwifery practices in total, expecting to recruit 4-5 respondents from each practice.

The study website included the baseline questionnaire (T0). Respondents could choose their own username and password and had to report their email address when signing up for the study. This way we could easily remove respondents with multiple identities from further analyses. Before providing informed consent, pregnant women were informed about the 3 study conditions and received information about the objectives of the study, the randomization procedure, and the incentive of a $€ 10$ voucher when respondents completed all questionnaires and institutional affiliations ("This research is conducted by the Dutch Institute for Alcohol Policy [STAP] and Maastricht University"). After providing online informed consent, eligible women gained access to the baseline questionnaire. Blinding of respondents was not possible because they had to take notice of whether they did or did not receive additional counselling from their midwife (after the baseline questionnaire) or tailored feedback via the computer (during the baseline questionnaire).

At both 3 and 6 months after the baseline questionnaire, all participants received an invitation by email (followed by 2 reminders after 2 and 4 weeks) for the first follow-up questionnaire ( $\mathrm{T} 1$ and $\mathrm{T} 2$, respectively). 
Nonrespondents after 2 reminders were contacted by telephone to collect their data.

\section{Interventions}

The health counselling and computer-tailoring interventions were both based on the I-Change model (De Vries, et al., 2003), a theoretical model incorporating concepts from several social cognitive models, such as the Transtheoretical model (Prochaska \& Velicer, 1997) and the Theory of Planned Behaviour (Ajzen, 1985). The I-Change model distinguishes 3 phases of health behaviour change (awareness, motivation, and action) and has been used successfully for developing various health promoting interventions, such as prenatal smoking cessation (De Vries, et al., 2006a), smoking cessation (E.S. Smit, et al., 2012; Te Poel, et al., 2009; Elfeddali, et al., 2012), and increasing vegetable and fruit intake and physical activity (De Vries, et al., 2008).

\section{Health Counselling}

Midwives in the health-counselling condition received a brief manual explaining the health-counselling protocol and an intervention card with questions for the clients. On this intervention card, midwives could record the dates of the health counselling sessions and the clients' answers to the midwife's questions. Midwives received 3 hours of training on how to provide the health counselling. This training was given either at the research institute of the first author or at the practice of the participating midwife. The materials and training were based on earlier work on tobacco and pregnancy (De Vries, et al., 2003).

The health-counselling protocol consisted of 7 steps which were addressed in 3 feedback sessions. Feedback session 1, approximately 2 weeks after baseline assessment, consisted of 5 steps taking approximately 10 minutes of the initial consultation (Feedback 1-health counselling). In step 1, the midwife assessed the amount and frequency of alcohol use of the pregnant woman before and during pregnancy, of her partner during pregnancy, and the pregnant woman's motivation 
to stop drinking alcohol. In step 2, women strongly motivated to stop alcohol consumption during pregnancy were prompted to state the advantages of abstinence. Moderately or not motivated women were asked to report on their perceived disadvantages of drinking during pregnancy. The midwife then advised them to stop drinking alcohol. In step 3, the barriers for successful abstinence and the mobilization of social support were discussed. In step 4, a self-help guide, adapted from an intervention on smoking in pregnancy (De Vries, et al., 2006a), and relevant websites were mentioned. The midwife stimulated the pregnant woman to develop action plans for abstinence and coping with problems they might encounter when trying not to drink alcohol. If appropriate, access to alcohol addiction services was discussed. In step 5, women were asked to set a date for stopping their alcohol use (goal setting). Feedback session 2, approximately 8 weeks after baseline, consisted of step 6, which was addressed in approximately 1 minute (Feedback 2-health counselling). In this step, midwives again assessed the alcohol use of the pregnant women and asked her if she needed additional support for not drinking alcohol. Feedback session 3, approximately 14 weeks after baseline, consisted of step 7, which was also addressed in approximately 1 minute (Feedback 3-health counselling). In this step, midwives discussed alcohol use and its implications for breastfeeding.

\section{Computer Tailoring}

The computer-tailored intervention was developed using Tailorbuilder software (OSE, the Netherlands), a program which is specifically designed to develop Web-based computer-tailored interventions. Respondents in the computer-tailoring group received usual care from their midwife and computer-tailored feedback via the Internet, which was iterative and item-based (Strecher, Shiffman, \& West, 2006). Feedback 1, given immediately after baseline, consisted of 4 to 5 pages (Feedback 1-computer tailoring). This feedback was tailored to several respondent characteristics assessed in the baseline questionnaire: alcohol use, knowledge, risk perception, attitude, social influence, selfefficacy, intention, and action and coping plans. Specifically, the first feedback letter contained the recommendation of complete alcohol abstinence during pregnancy and information on possible consequences 
of prenatal alcohol use and the associated risk factors. In addition, feedback was provided on the respondent's risk perception of prenatal alcohol use; her attitude (perceived advantages and disadvantages toward prenatal alcohol use and alcohol abstinence; perceived social influence (not) to drink during pregnancy; self-efficacy to refrain from prenatal alcohol use in specific situations, including suggestions on how to cope with these situations; the extent to which respondents were planning to undertake specific actions (action plans) to abstain from prenatal alcohol use; and how to cope with certain difficult situations (coping plans), including the formulation of personal plans in the shape of if-then statements (Van Osch, et al., 2008). The second feedback letter, 6 weeks after baseline, included personalized information on the respondents' choice of characteristics assessed with the baseline questionnaire (e.g., risk perception or attitude; Feedback 2-computer tailoring). Depending on the number of characteristics chosen by the respondent, this feedback consisted of 1 or 2 pages. The third feedback letter, given immediately after T1, consisted of 3 to 4 pages of ipsative feedback tailored to changes in the respondent characteristics assessed at $\mathrm{T} 1 \mathrm{in}$ comparison to the baseline questionnaire (Feedback 3-computer tailoring). Feedback letters were visible on the computer screen and also sent to the respondent by email. Figure 5.1 shows an example of items regarding action plans to abstain from prenatal alcohol use. Figure 5.2 shows an example of a tailored feedback message. 


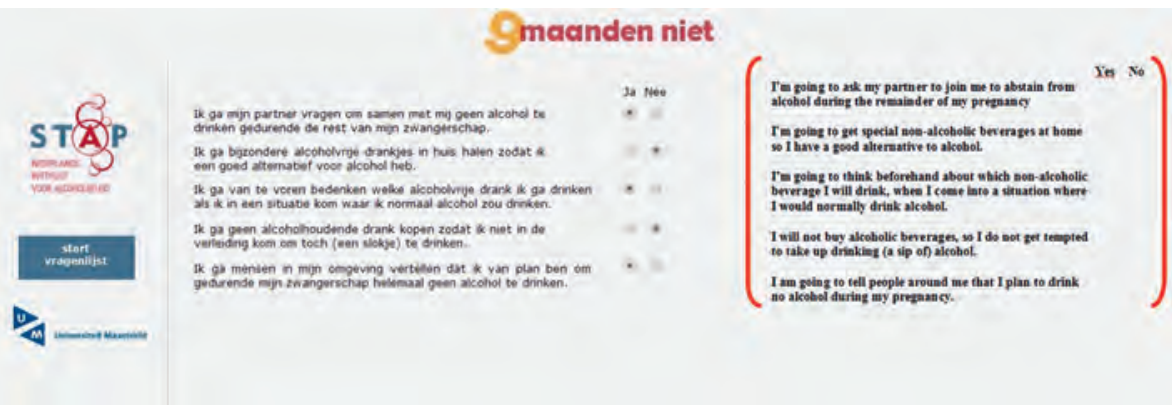

Figure 5.1 Screenshot and English translation of items regarding action plans to abstain from prenatal alcohol use.

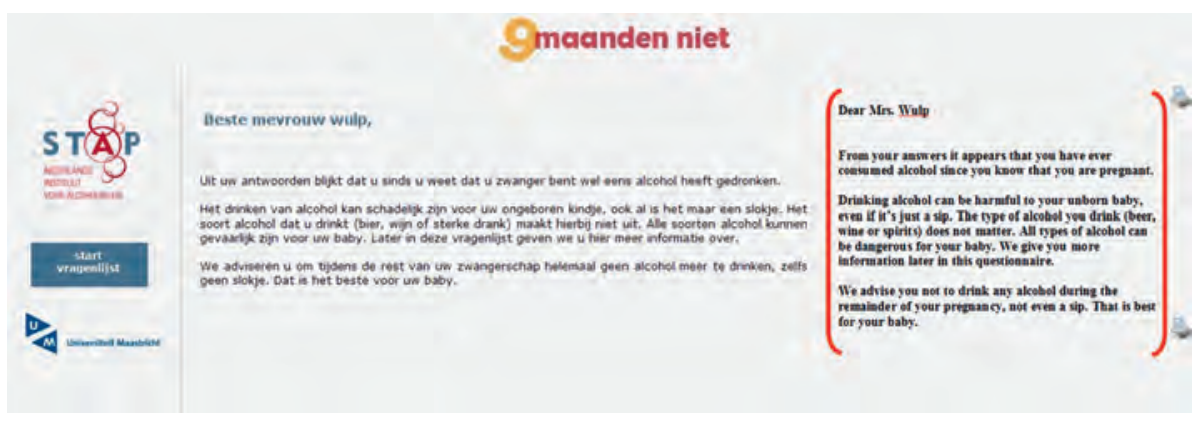

Figure 5.2 Screenshot and English translation of personal advice regarding prenatal alcohol use.

\section{Usual Care}

Midwives in the usual care group were instructed to give routine alcohol care. In-line with the national guidelines, midwives recommend complete alcohol abstinence to clients who are using alcohol in the initial consultation (De Jonge, 2005; De Boer \& Zeeman, 2008). In practice, not much time is spent on this nor is it common to provide additional counselling or other information (Van der Wulp, Hoving, \& de Vries, 2013). 


\section{Pretests of the Interventions}

The midwives' manual of the health counselling intervention was pretested among 5 midwives and the computer-tailoring intervention was pretested among 5 pregnant women using alcohol. The pretests yielded information about unclear questions and formulations in the manual and in the computer-tailoring intervention, which was used to improve the texts in the final versions of the health-counselling manual and computer-tailoring intervention.

\section{Measures}

\section{Baseline Questionnaire}

The baseline questionnaire required 15 minutes to complete, consisted of 92 questions, and was based on questionnaires in previous studies applying the I-Change model (Dijkstra, De Vries, \& Royackers, 1998a; Hoving, Mudde, \& de Vries, 2007a; Hoving, et al., 2010). Questions assessed alcohol use in pregnancy (average alcohol use, binge drinking, and risky drinking), predisposing factors (drinking behaviour before pregnancy, demographics, and smoking behaviour), awareness factors (risk perception), and motivational factors (attitude, social influences, and self-efficacy).

Average alcohol consumption during pregnancy was assessed with the 5-item Dutch Quantity-Frequency-Variability (QFV) questionnaire (Lemmens, Tan, \& Knibbe, 1992). Respondents selected the type of alcoholic drinks that they had consumed since the beginning of their pregnancy, such as beer, wine, or cocktails. Respondents were asked to indicate how many working days (Monday to Thursday) on average they had consumed this type of alcohol since the beginning of their pregnancy. Additionally, they were asked to indicate the quantity (number of sips, glasses, or bottles) they had usually consumed of this type of alcohol on these occasions. Similar questions were asked concerning alcohol consumption during weekend days (Friday to Sunday). The average number of drinking working days multiplied by the average alcohol consumption per working day plus the average 
number of drinking weekend days multiplied by the average alcohol consumption per weekend day comprised the average weekly alcohol consumption during pregnancy.

We assessed 2 types of hazardous prenatal drinking behaviour because previous research on alcohol use in pregnancy often used either of these types (Jones, Bailey, \& Sokol, 2013). First, binge drinking in pregnancy was assessed by asking respondents if they ever had 4 or more standard glasses of alcohol (consisting of 10 grams of pure alcohol) on 1 day since they knew they were pregnant $(0=$ no; $1=y e s)$. Second, risky drinking during pregnancy was assessed with the validated T-ACE screening tool (Sokol, Martier, \& Ager, 1989) (0=not risky drinking; 1=risky drinking).

Average alcohol consumption before pregnancy was also assessed with the QFV and was calculated similarly to the average weekly alcohol consumption during pregnancy.

Demographic information regarding age, education (primary school/ basic vocational school, secondary vocational school/high school degree, higher vocational school/college degree/university degree), income (0.5 or less; $0.5-2$; more than 2 times net Dutch median household income), and relationship status ( $0=$ no steady partner; $1=$ steady partner) was assessed. Pregnancy-related questions included number of weeks pregnant, number of prior pregnancies $(0,1,2$, or more than 2 prior pregnancies), and experience with complications in a previous pregnancy ( $0=$ no; $1=y e s)$.

Respondents' smoking behaviour (in number of cigarettes per day) during and before pregnancy was assessed ("On average, how many cigarettes do you smoke per day").

Risk perception was operationalized with perceived likelihood that the baby would experience harm ("If I drink alcohol in pregnancy, the chance that it damages my baby is..."; $1=$ very low; $5=$ very high) and perceived severity of that harm ("If I drink alcohol in pregnancy, the severity of the damage due to that alcohol is...”; $1=$ not serious; $5=$ very serious) resulting from alcohol use during pregnancy. 
Attitude concerning alcohol use in pregnancy was assessed using 12 items (-2=disagree; $2=$ agree). A factor analysis using varimax rotation revealed 2 components: advantages (pros) and disadvantages (cons) of not drinking alcohol in pregnancy. Pros were assessed by 6 items $(\alpha=.75)$, such as "If I do not drink any alcohol in pregnancy, it is better for my baby's health." Cons of not drinking alcohol in pregnancy were assessed by another 6 items ( $\alpha=.82)$, such as "If I do not drink any alcohol in pregnancy, I feel more tense."

Social support to abstain from alcohol in pregnancy was assessed with 3 items on a 5 -point scale $(\alpha=.91)$ asking respondents whether they were supported by their partner, mother, and friends to abstain from alcohol in pregnancy, such as "My partner supports me not to drink alcohol in pregnancy" (-2=totally disagree; $2=$ totally agree).

Self-efficacy toward alcohol abstinence in pregnancy in social situations was assessed by 6 items on a 5 -point scale $(\alpha=.90)$, such as "How easy it is for you to abstain from alcohol when your partner drinks alcohol" (-2=very difficult; $2=$ very easy).

\section{Follow-Up Questionnaires}

Posttest drinking behaviour ("Have you had at least 1 sip of alcohol since the previous questionnaire?"; $0=$ no; $1=y e s)$ and average weekly alcohol consumption since the previous questionnaire (assessed with the QFV) were assessed at $\mathrm{T} 1$ and at $\mathrm{T} 2$.

\section{Analyses}

The respondents who had had a miscarriage since the baseline were excluded from the analyses. The other respondents who did not complete the posttest questionnaire remained in the dataset and were considered as missing at random (MAR). Because respondents were nested in midwifery practices, all analyses were conducted using a mixed model analysis (SPSS v19). 
To test whether conditions differed with regard to drop out, logistic mixed model analyses of dropout at $\mathrm{T} 1$ and $\mathrm{T} 2$ ( $0=$ no drop out; $1=$ drop out due to miscarriage, being unreachable, or being no longer interested to participate) were conducted with condition, age, education, steady partner, number of prior pregnancies, alcohol use before pregnancy, and smoking as independent variables.

To check for potentially confounding variables, univariate linear regressions with condition as predictor were performed and tested whether baseline characteristics of respondents differed between the 3 conditions.

In a set of multiple logistic mixed model analyses, we investigated the effect of condition in addition to the effect of covariates (concepts of the I-Change model) on posttest drinking behaviour at $\mathrm{T} 1$ and $\mathrm{T} 2(\mathrm{O}=\mathrm{not}$ drinking; $1=$ still drinking). Significant interactions of covariates with condition were detected in a set of multiple logistic regression analyses conducted in a top-down procedure in which the least significant interaction, with $\mathrm{P}>05$, was omitted from a subsequent analysis. Significant main effects of covariates were also detected in a set of multiple logistic regression analyses conducted in a top-down procedure in which the least significant main effect, with $\mathrm{P}>.05$, was omitted from a subsequent analysis. If there were no significant interaction effects with condition, we conducted a final multiple logistic regression with condition and the significant main effects of covariates and drinking behaviour at $\mathrm{T} 1$ and $\mathrm{T} 2$ as outcome variable. If there were significant interaction effects with condition, we probed the interaction to understand the role of condition. Following Hayes and Matthes (2009), we used the pick-a-point approach and tested whether condition was significant at 3 points on the moderator variable (1 standard deviation below average, average, and 1 standard deviation above average).

For respondents who were still drinking alcohol at $\mathrm{T} 1$ and $\mathrm{T} 2$, we tested the effect of condition in addition to the effect of covariates on the reduction of alcohol use. We performed similar sets of analyses as described previously using multiple linear mixed model analysis to 
assess the effect of condition in addition to the effect of confounding and moderating variables on average weekly alcohol consumption. Because of a right-skewed distribution (relatively few respondents had a high average weekly alcohol consumption), a transformation by the natural logarithm was applied to the average weekly alcohol consumption at T1 and $\mathrm{T} 2$.

Finally, sensitivity analyses were conducted to test the robustness of the MAR assumption for the first hypothesis. These sensitivity analyses comprised the elaboration of 3 scenarios. First, all missing values were considered as still drinking alcohol; second, all missing values were considered as having stopped drinking alcohol. The third scenario entailed that women in the health-counselling condition who had quit alcohol were as likely as those who had not quit alcohol to return the follow-up questionnaire (e.g., because of their connection with their midwife) whereas women in the computer tailoring and usual care conditions who had quit alcohol were twice as likely to return the follow-up questionnaire than those who had not quit alcohol (e.g., because they wanted the researchers to know they had been successful). The robustness of the MAR assumption is supported when outcomes of these scenarios (including significant covariates) are similar to the outcomes of the analyses without the imputation of the missing values (Enders, 2010).

\section{Results}

\section{Recruitment Results}

The baseline questionnaire was completed by 393 respondents. In total, 135 respondents were assigned to the health-counselling condition, 116 respondents to the computer-tailoring condition, and 142 respondents to the usual care condition (Figure 5.3). These numbers varied slightly per condition because midwives in the 3 conditions yielded a slightly different number of participating women. 


\section{Selective Dropout}

A total of 99 of 135 health-counselling respondents (73.3\%), 77 of 116 computer-tailoring respondents (66.4\%), and 108 of 142 usual care respondents $(76.1 \%)$ completed T1. Multilevel logistic regression analysis with dropout (no/yes) at $\mathrm{T} 1$ as outcome variable and condition, age, education, steady partner, number of prior pregnancies, alcohol use before pregnancy, and smoking as covariates showed a significant fixed effect for having a steady partner. Respondents without a steady partner (OR 0.497, 95\% CI 0.305-0.809; $\mathrm{P}=.005$ ) were significantly less likely to drop out at T1. Condition and random effects were not significant.

A total of 86 of 135 health-counselling respondents (63.7\%), 68 of 116 computer-tailoring respondents (58.6\%), and 93 of 142 usual care respondents $(65.5 \%)$ completed the T2 questionnaire. Multilevel logistic regression analysis with dropout (no/yes) at $\mathrm{T} 2$ as outcome variable did not show a significant effect for condition or any other factor.

\section{Sample Characteristics}

Analyses on sample characteristics were conducted on the baseline characteristics of all respondents except 44 respondents with a miscarriage (114 health-counselling respondents, 111 computer-tailoring respondents, 124 usual care respondents; see Table 5.1). This sample had a mean age of 32.6 (SD 4.23) years. Most women were highly educated and had a medium income. The respondents were, on average, nearly 8 weeks pregnant, had been drinking almost 6 standard drinks of alcohol per week prior to the pregnancy, and drank 1 standard drink of alcohol per week during pregnancy. 


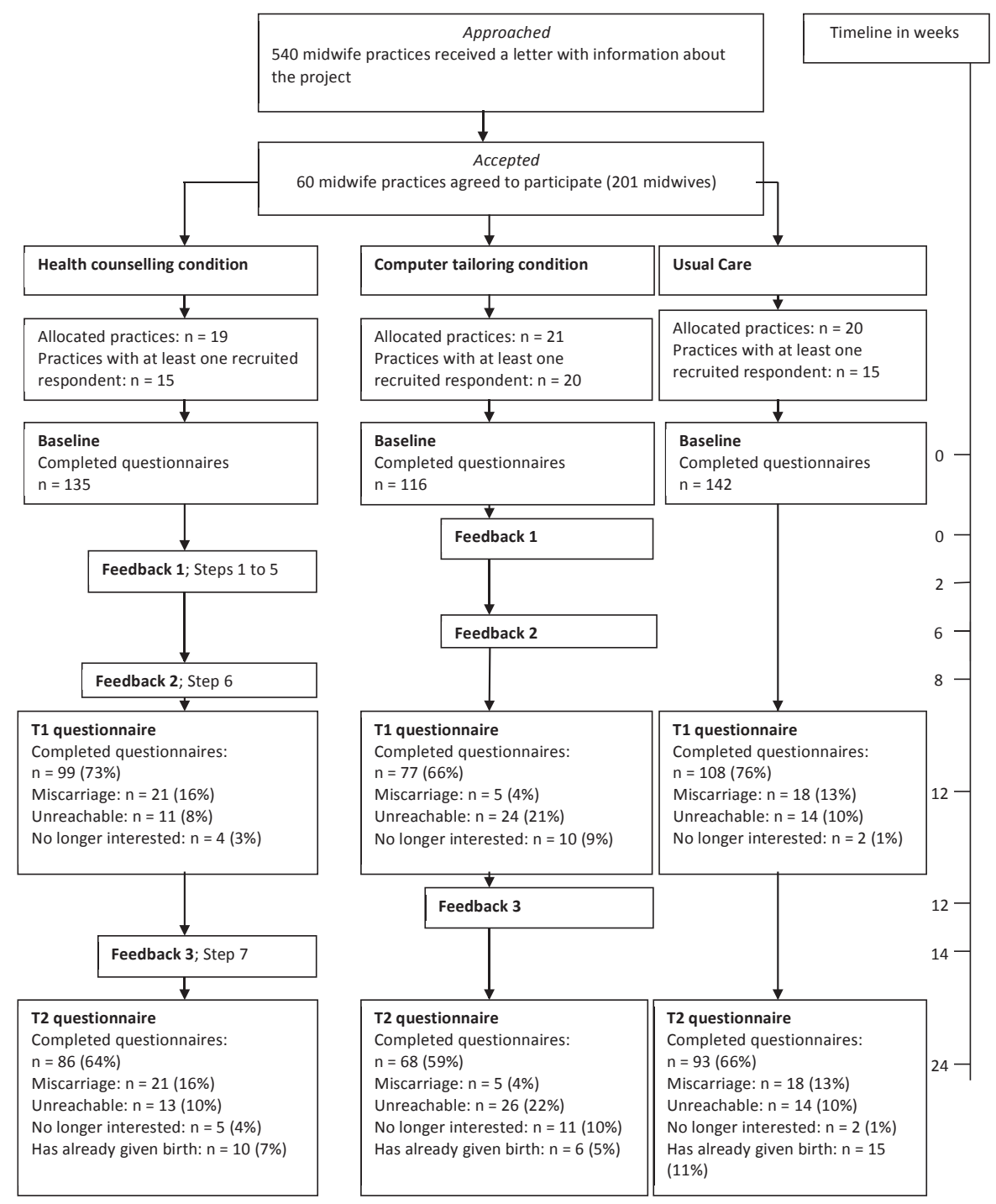

Figure 5.3 Flowchart of cluster randomized trial testing the effectiveness of health counselling and computer tailoring compared to usual care

Multilevel analyses with sample characteristics as outcome variables and condition as predictor showed that computer-tailoring respondents drank significantly less alcohol before pregnancy compared to usual care respondents, and that health-counselling and computer-tailoring respondents smoked cigarettes more often compared to usual care 
respondents. Thus, alcohol use before pregnancy and smoking were considered potentially confounding variables in subsequent analyses. At the level of midwifery practices, respondents differed significantly with regard to educational level, income, and number of weeks of pregnancy indicating the necessity of a multilevel approach in the subsequent analyses.

\section{Drinking Behaviour at T1 and T2}

Our first hypothesis stated that women receiving health counselling or computer tailoring would be more likely to stop using alcohol in pregnancy compared to women receiving usual care at T1 and at T2. The results at T1 did not support our hypothesis. At T1, 64 of 99 healthcounselling respondents (65\%), 54 of 77 computer-tailoring respondents $(70 \%)$, and 49 of 108 usual care respondents (45.4\%) had refrained from alcohol. These differences were not significant $(\mathrm{P}=.79$ for health counselling vs usual care; $\mathrm{P}=.15$ for computer tailoring vs usual care; $\mathrm{P}=.23$ for health counselling vs computer tailoring).

At T2, 62 of 86 health-counselling respondents (72\%), 53 of 68 computertailoring respondents (78\%), and 51 of 93 of the usual care respondents (55\%) had refrained from alcohol. Table 5.2 presents the final model of the multilevel multiple logistic regression analyses with drinking behaviour at T2 as outcome variable. The effects of the covariates (i.e., alcohol use before pregnancy, smoking, age, education, perceived likelihood and perceived severity of risk due to prenatal alcohol use, pros and cons of not drinking alcohol in pregnancy, social support to abstain from alcohol in pregnancy, and social self-efficacy) were tested for significance and, if not significant, removed from the analysis model. The final analysis model showed that computer-tailoring respondents had refrained from alcohol significantly more often compared to usual care respondents, supporting our first hypothesis. However, the difference between health counselling and usual care respondents was not significant $(\mathrm{P}=.26)$. Moreover, the difference between computertailoring and health-counselling respondents was not significant either $(\mathrm{P}=.32)$. 


\section{Average Weekly Alcohol Consumption at T1 and T2}

Our second hypothesis stated that women who continued their alcohol use would be more successful in reducing their alcohol consumption after receiving health counselling or computer tailoring at $\mathrm{T} 1$ and at $\mathrm{T} 2$. This hypothesis was not supported at T1. Only considering respondents who had not stopped drinking alcohol $(\mathrm{n}=35$ in health counselling; $\mathrm{n}=23$ in computer tailoring; $\mathrm{n}=59$ in usual care), health-counselling respondents drank on average 0.56 standard drinks of alcohol per week (SD 0.91), computer-tailoring respondents drank 0.27 units (SD 0.17), and usual care respondents drank 0.51 units (SD 0.54). These differences were not significant $(\mathrm{P}=.58$ for health counselling vs usual care; $\mathrm{P}=.23$ for computer tailoring vs usual care; $\mathrm{P}=.49$ for health counselling vs computer tailoring).

Table 5.1 Baseline sample characteristics of Dutch pregnant women using alcohol $(\mathrm{N}=349)$.

\begin{tabular}{|c|c|c|c|c|c|c|}
\hline Characteristic & $\begin{array}{l}\text { Overall } \\
\text { sample } \\
(\mathrm{N}=349)\end{array}$ & $\begin{array}{l}\text { Health } \\
\text { counselling } \\
(\mathrm{n}=114)\end{array}$ & $\begin{array}{l}\text { Computer } \\
\text { tailoring } \\
(\mathrm{n}=111)\end{array}$ & $\begin{array}{l}\text { Usual care } \\
(\mathrm{n}=124)\end{array}$ & $\begin{array}{l}\text { Condition } \\
\text { effect, P }\end{array}$ & $\begin{array}{l}\text { Random } \\
\text { effect of } \\
\text { mid-wifery } \\
\text { practices, P }\end{array}$ \\
\hline $\begin{array}{l}\text { Age (years), } \\
\text { mean (SD) }\end{array}$ & $32.56(4.20)$ & $31.75(4.37)$ & $32.31(4.22)$ & $33.53(3.85)$ & .17 & .07 \\
\hline $\begin{array}{l}\text { Educational } \\
\text { level, } \mathrm{n}(\%)\end{array}$ & & & & & .15 & .02 \\
\hline Low & $9(2.6)$ & $5(4.5)$ & $1(0.9)$ & $3(2.4)$ & & \\
\hline Medium & $108(31.2)$ & $47(42.0)$ & $41(36.9)$ & $20(16.3)$ & & \\
\hline High & $229(66.2)$ & $60(53.6)$ & $69(62.2)$ & $100(81.3)$ & & \\
\hline Income, $\mathrm{n}(\%)$ & & & & & .93 & .03 \\
\hline Low & $35(11.3)$ & $14(13.9)$ & $9(9.0)$ & $12(11.0)$ & & \\
\hline Medium & $170(54.8)$ & $56(55.4)$ & $62(62.0)$ & $52(47.7)$ & & \\
\hline High & $105(33.9)$ & $31(30.7)$ & $29(29.0)$ & $45(41.3)$ & & \\
\hline $\begin{array}{l}\text { Steady } \\
\text { partner, n (\%) }\end{array}$ & $198(56.7)$ & $73(64.0)$ & $66(59.5)$ & $59(47.6)$ & .17 & .33 \\
\hline $\begin{array}{l}\text { Number } \\
\text { of prior } \\
\text { pregnancies, } \\
\mathrm{n}(\%)\end{array}$ & & & & & .33 & .14 \\
\hline 0 & $150(43.0)$ & $51(44.7)$ & 37 (33.3) & $62(50.0)$ & & \\
\hline 1 & $113(32.4)$ & $30(26.3)$ & $44(39.6)$ & $39(31.5)$ & & \\
\hline 2 & $56(16.0)$ & $23(20.2)$ & $16(14.4)$ & $17(13.7)$ & & \\
\hline$>2$ & $30(8.6)$ & $10(8.8)$ & $14(12.6)$ & $6(4.8)$ & & \\
\hline $\begin{array}{l}\text { Number } \\
\text { of weeks } \\
\text { pregnant, } \\
\text { mean (SD) }\end{array}$ & $7.87(1.96)$ & $7.96(1.81)$ & $7.73(2.06)$ & $7.92(1.99)$ & .72 & .02 \\
\hline
\end{tabular}


Table 5.1 (continued). Baseline sample characteristics of Dutch pregnant women using alcohol $(\mathrm{N}=349)$.

\begin{tabular}{|c|c|c|c|c|c|c|}
\hline Characteristic & $\begin{array}{l}\text { Overall } \\
\text { sample } \\
(\mathrm{N}=349)\end{array}$ & $\begin{array}{l}\text { Health } \\
\text { counselling } \\
(\mathrm{n}=114)\end{array}$ & $\begin{array}{l}\text { Computer } \\
\text { tailoring } \\
(\mathrm{n}=111)\end{array}$ & $\begin{array}{l}\text { Usual } \\
\text { care } \\
(\mathrm{n}=124)\end{array}$ & $\begin{array}{l}\text { Condition } \\
\text { effect, P }\end{array}$ & $\begin{array}{l}\text { Random } \\
\text { effect of } \\
\text { mid-wifery } \\
\text { practices, } P\end{array}$ \\
\hline $\begin{array}{l}\text { Experienced } \\
\text { complications } \\
\text { in previous } \\
\text { pregnancy, n (\%) }\end{array}$ & $76(22.4)$ & 23 (20.9) & $26(23.9)$ & $27(22.3)$ & .87 & .92 \\
\hline $\begin{array}{l}\text { Standard alcohol } \\
\text { drinks per } \\
\text { week during } \\
\text { pregnancy, mean } \\
\text { (SD) }\end{array}$ & $1.13(2.87)$ & $1.44(3.33)$ & $1.21(3.14)$ & $0.76(2.02)$ & .23 & .72 \\
\hline $\begin{array}{l}\text { Binge drinkers } \\
\text { during } \\
\text { pregnancy, }{ }^{b} \mathrm{n}(\%)\end{array}$ & $4(1.2)$ & $3(2.7)$ & $0(0)$ & $1(0.8)$ & .17 & - \\
\hline $\begin{array}{l}\text { Risky drinkers } \\
\text { (T-ACE positive), } \\
\text { n (\%) }\end{array}$ & $198(57.4)$ & $73(64.6)$ & $55(50.9)$ & $70(56.5)$ & .13 & .93 \\
\hline $\begin{array}{l}\text { Standard alcohol } \\
\text { drinks per } \\
\text { week before } \\
\text { pregnancy, mean } \\
\text { (SD) }\end{array}$ & $5.83(7.35)$ & $5.61(8.88)$ & $\begin{array}{l}4.53 a \\
(4.61)\end{array}$ & $7.18(7.59)$ & .06 & .62 \\
\hline $\begin{array}{l}\text { Smokes in } \\
\text { pregnancy, }{ }^{\mathrm{b}} \mathrm{n}(\%)\end{array}$ & 69 (20.2) & $30(27.0)^{a}$ & $25(23.4)^{\mathrm{a}}$ & $14(11.3)$ & .01 & - \\
\hline
\end{tabular}

a Indicates significant difference compared to usual care.

b Single-level analyses were conducted on the characteristics binge drinking and smoking during pregnancy because in the multilevel analyses, the estimates of the variances of the random effects were 0 and the Hessian matrices were not positive definite.

Table 5.2 Final model of the multilevel multiple logistic regression analysis concerning drinking behavior at $\mathrm{T} 2(\mathrm{~N}=241){ }^{\mathrm{a}}$

\begin{tabular}{lllllll}
\hline Fixed effects & $\begin{array}{l}\text { Estimated } \\
\text { variance }\end{array}$ & $\mathrm{B}$ & $\mathrm{SE}$ & $\mathrm{OR}$ & $95 \% \mathrm{CI}$ & $\mathrm{P}$ \\
\hline $\begin{array}{l}\text { Health } \\
\text { counselling }\end{array}$ & & 0.52 & 0.46 & 1.68 & $0.68,4.18$ & .26 \\
$\begin{array}{l}\text { Computer } \\
\text { tailoring }\end{array}$ & & 1.02 & 0.49 & 2.77 & $1.05,7.34$ & .04 \\
$\quad$ Age & -0.11 & 0.05 & 0.89 & $0.82,0.98$ & .01 \\
$\begin{array}{l}\text { Perceived } \\
\text { likelihood }\end{array}$ & 0.48 & 0.16 & 1.61 & $1.18,2.19$ & .003 \\
$\quad$ & & 0.53 & 0.18 & 1.69 & $1.19,2.41$ & .004 \\
$\begin{array}{l}\text { Self-efficacy } \\
\text { Random effect }\end{array}$ & 0.40 & & 0.36 & & & .13 \\
\hline
\end{tabular}

a 6 respondents were lost because they had not filled in the questions about self-efficacy in social situations.

${ }^{\mathrm{b}}$ Usual care is the reference category. 
Our second hypothesis was partially supported at T2. Table 5.3 summarizes the results of the multilevel multiple linear regression analyses with average weekly alcohol consumption at T2 for those respondents who had not stopped drinking alcohol $(n=23$ in health counselling; $n=15$ in computer tailoring; $n=41$ in usual care). Healthcounselling respondents drank on average 0.77 standard drinks of alcohol per week (SD 1.36), computer-tailoring respondents drank 0.35 units (SD 0.31), and usual care respondents drank 0.48 units (SD 0.54). Due to the significant interaction effects of computer tailoring with alcohol use before pregnancy and computer tailoring with social support, the main effect of computer tailoring could not be interpreted (see Table 5.3). To understand for which persons computer tailoring had a significant effect on the reduction of their alcohol use and for which persons computer tailoring was not significant, we probed the interactions by means of the pick-a-point approach. This showed that computer tailoring significantly reduced the alcohol use at T2 compared to usual care among respondents who had an average $(\mathrm{P}=.007)$ or 1 standard deviation below the average of alcohol use before pregnancy $(\mathrm{P}<.001)$, but not among respondents 1 standard deviation above the average of alcohol use before pregnancy $(\mathrm{P}=.57)$. In addition, computer tailoring significantly reduced alcohol use at $\mathrm{T} 2$ compared to usual care among respondents average $(\mathrm{P}=.03)$ or 1 standard deviation below the average of social support $(\mathrm{P}=.002)$, but not among respondents 1 standard deviation above the average of social support $(\mathrm{P}=.87)$. The analyses additionally showed that health counselling was not significant.

\section{Sensitivity Analyses for Missing-at-Random Assumption of Posttest Drinking Behaviour (No/Yes)}

We conducted sensitivity analyses for drinking behaviour at T2 because with this outcome variable computer tailoring differed significantly from usual care. Scenario 1 entailed that all missing values were replaced with 1 (still drinking alcohol at T2). In scenario 1, 62 of 135 health-counselling respondents (45.9\%), 53 of 116 computer-tailoring respondents (45.7\%), and 51 of 142 usual care respondents (35.9\%) refrained from alcohol. A 
multiple logistic regression analysis with drinking behaviour 6 months after baseline according to scenario 1 as outcome variable showed that more computer-tailoring respondents refrained from alcohol than usual care respondents $(\mathrm{P}=.06)$. The difference between health counselling and usual care was not significant $(\mathrm{P}=.46)$.

Scenario 2 entailed that all missing values were replaced by 0 (not drinking alcohol at T2). In scenario 2, 111 of 135 health-counselling respondents (82.2\%), 101 of 116 computer-tailoring respondents (87.1\%), and 100 of 142 usual care respondents (70.4\%) refrained from alcohol. A multiple logistic regression analysis with drinking behaviour 6 months after baseline according to scenario 2 as outcome variable showed that significantly more computer-tailoring respondents refrained from alcohol than usual care respondents $(\mathrm{P}=.04)$. The difference between health counselling and usual care was not significant $(\mathrm{P}=.35)$.

In scenario 3, 36 out 49 missing values in the health-counselling condition (73\%) were randomly replaced by 0 (not drinking alcohol at T2) and 13 out 49 missing values (27\%) by 1 (still drinking alcohol at $\mathrm{T} 2)$; in the computer-tailoring condition, 19 of 48 missing values (40\%) were randomly replaced by 0 and 29 of 48 missing values (60\%) by 1 ; in the usual care condition, 14 of 49 missing values (29\%) were randomly replaced by 0 and 35 of 49 missing values (71\%) by 1 . A multiple logistic regression analysis with drinking behaviour 6 months after baseline according to scenario 3 as outcome variable showed that significantly more computer-tailoring respondents (72/116, 62.1\%) and healthcounselling respondents (98/135 respondents, 72.6\%) refrained from alcohol than usual care respondents (65/142 respondents, 45.8\%; $\mathrm{P}=.04$ and $\mathrm{P}=.01$, respectively).

\section{Discussion}

The goal of this study was to test the effectiveness of 2 different brief interventions to reduce prenatal alcohol use: a health counselling and a computer-tailored intervention in comparison with usual care. We 
hypothesized that women receiving a newly developedhealth counselling or computer-tailored intervention were more likely to stop (hypothesis 1) and reduce (hypothesis 2) their prenatal alcohol use compared to women receiving usual care. This effect study showed that after 6 months and 3 feedback letters, the computer-tailoring program was effective in stopping prenatal alcohol use and in reducing it under certain conditions compared to usual care; the health-counselling protocol was not.

Table 5.3 Final model of the multilevel multiple linear regression analysis with the natural logarithm of average alcohol consumption at T2 as outcome variable among alcohol users only $(\mathrm{N}=73) .^{\mathrm{a}}$

\begin{tabular}{|c|c|c|c|c|c|}
\hline Fixed effects & $\begin{array}{l}\text { Estimated } \\
\text { variance }\end{array}$ & B & SE & $95 \% \mathrm{CI}$ & $\mathrm{P}$ \\
\hline $\begin{array}{l}\text { Health } \\
\text { counselling }^{\mathrm{b}}\end{array}$ & & -1.11 & 0.92 & $-2.94,0.72$ & .23 \\
\hline $\begin{array}{l}\text { Computer } \\
\text { tailoring }\end{array}$ & & 6.41 & 1.75 & $2.92,9.90$ & $<.001$ \\
\hline Not smoking & & -1.23 & 0.40 & $-2.03,-0.43$ & .003 \\
\hline $\begin{array}{l}\text { Alcohol use } \\
\text { before pregnancy }\end{array}$ & & 0.00 & 0.03 & $-0.06,0.06$ & .95 \\
\hline Social support & & 0.16 & 0.16 & $-0.16,0.47$ & .34 \\
\hline $\begin{array}{l}\text { Health } \\
\text { counselling }{ }^{\mathrm{b} *} \\
\text { alcohol use } \\
\text { before pregnancy }\end{array}$ & & -0.05 & 0.05 & $-0.15,0.05$ & .32 \\
\hline $\begin{array}{l}\text { Computer } \\
\text { tailoring }{ }^{\mathrm{b}} * \\
\text { alcohol use } \\
\text { before pregnancy }\end{array}$ & & -0.43 & 0.12 & $-0.67,-0.18$ & .001 \\
\hline $\begin{array}{l}\text { Health } \\
\text { counselling } * \\
\text { social support }\end{array}$ & & 0.39 & 0.24 & $-0.08,0.87$ & .10 \\
\hline $\begin{array}{l}\text { Computer } \\
\text { tailoring }{ }^{\mathrm{b}} * \text { social } \\
\text { support }\end{array}$ & & -1.38 & 0.35 & $-2.08,-0.67$ & $<.001$ \\
\hline Random effect & 0 & & 0 & & \\
\hline
\end{tabular}

The ineffectiveness of the newly developed health-counselling protocol was inconsistent with the significant effects of health counselling interventions in the related field of smoking cessation in pregnancy (De Vries, et al., 2006a; Bolman, de Vries, \& van Breukelen, 2002; Pieterse, 
et al., 2001). One shortcoming of this effectiveness study was the lack of statistical power. The power was planned to be .80 but turned out to be approximately .50 due to a larger intraclass correlation and a higher percentage of usual care participants who continued drinking than estimated beforehand. Although this amount of power was sufficient to show a significant effect of the computer-tailoring intervention at 6 months after baseline, the $20 \%$ difference between health-counselling and usual care respondents who stopped drinking alcohol at T1 and the $17 \%$ difference at T2 were not significant. It is unclear whether health counselling would have been found effective with more statistical power.

A second shortcoming of this study was the suboptimal implementation of the health counselling intervention by the midwives. Our process evaluation showed that the health-counselling midwives had given counselling less extensively as they were trained (Van der Wulp, Hoving, \& de Vries, 2014b). For example, the majority of midwives did not offer the second and third counselling sessions because they thought their clients did not need or like to receive this successive counselling. Also, in the related field of smoking cessation in pregnancy, results were ineffective when health professionals were found to lack skills to implement their tasks as intended (De Vries, et al., 2006a; DePue, et al., 2002). A review on the barriers and facilitators of the effective implementation of brief interventions for alcohol misuse does show that effective implementation requires adequate training in which practitioners obtain sufficient confidence and knowledge to address drinking behaviour without being worried to upset patients (Johnson, et al., 2011).

Both shortcomings imply that the ineffectiveness of the health counselling intervention may not be simply due to an unsuccessful protocol. Perhaps the health-counselling protocol would have led to significant effects on the reduction of prenatal alcohol use with a higher amount of power and a better implementation. Future researchers testing an intervention to reduce prenatal alcohol use are recommended to take these issues into consideration (e.g., Wilson, et al., 2012). 
This is the first study showing that computer tailoring is effective in reducing prenatal alcohol use. The presently reported effect is in-line with previous studies showing how computer tailoring can effectively change health-related behaviours, such as smoking (Dijkstra, et al., 1998a), vegetable and fruit intake (Oenema, et al., 2005), and alcohol use (Boon, et al., 2011). This computer-tailoring intervention is a promising method to reduce prenatal alcohol use. The high percentage of pregnant women using alcohol in the Netherlands (Health Council of the Netherlands, 2005) shows that alternatives to usual care are needed. Previous research has shown that pregnant women are reluctant to disclose their alcohol use to health professionals (e.g., Van der Wulp, et al., 2013). Because computer tailoring preserves a person's anonymity (Cline \& Haynes, 2001), computer tailoring may be an attractive intervention for these women. Moreover, the implementation of computer tailoring is not affected by barriers to the effective implementation of health counselling interventions, such as lack of resources, training, and support from management, as well as workload of practitioners providing health counselling (F. Smit, et al., 2011). Finally, previous research has shown that computer tailoring can be cheaper than a health counselling intervention (F. Smit, et al., 2011; E.S. Smit, et al., 2013b) and may, therefore, be a cost-effective method to decrease prenatal alcohol use, although additional research is needed to support this supposition.

A major strength of the present study was the use of a theoretical framework, which has been previously used in interventions for a variety of health behaviours (e.g., E.S. Smit, et al., 2012; De Vries, et al., 2006a; Van Stralen, et al., 2011). In addition, both interventions used 3 feedback moments. Previous research on computer tailoring has shown that multiple feedback moments are likely to be more effective than a single feedback moment (Brug, et al., 1998; Dijkstra, et al., 1998b; Elfedalli, et al., 2012). More research is needed to explore the optimal number of feedback moments for both computer tailoring and for health counselling interventions. A limitation of the present study is the high percentage of dropout of respondents, especially in the computertailoring condition. Nevertheless, our sensitivity analyses show that the 
effectiveness of the computer-tailoring intervention is robust despite this high percentage of dropout. Another potential limitation is the reliance on self-report of alcohol use. Although the QFV is considered reasonably reliable (Lemmens, et al., 1992), the use of more objective assessments, such as urine tests, may have yielded different results. Nevertheless, self-report methods of drinking (e.g., QFV, the Alcohol Timeline Followback ; Sobell \& Sobell, 1992) have been used in many studies on human drinking behaviour because they are inexpensive, noninvasive, and acceptable to respondents (Del Boca \& Darkes, 2003). Moreover, it is likely that the potential underreporting of alcohol use has occurred to an equal extent in the experimental and control conditions, upholding the effectiveness of computer tailoring. Finally, it was not possible to compare the effectiveness of computer tailoring with health counselling due to various differences in the set-ups of the interventions, including the anonymity of the respondents and the timing of the feedback. Only when the set-ups of the interventions are identical, future research will be able to compare the effectiveness of computer tailoring with health counselling.

To conclude, this research tested the effectiveness of two newly developed interventions to reduce prenatal alcohol use. Despite previous studies showing effects of health counselling in reducing prenatal alcohol use, our health counselling intervention was not effective. Future studies testing health counselling interventions are recommended to invest more in recruitment of pregnant women and implementation by health care providers. Our computer-tailoring intervention was effective in stopping and reducing prenatal alcohol use at 6 month follow-up. A costeffectiveness study is recommended to determine the costs and effects associated with this intervention and compare them with the costs and effects of other interventions and/or usual care. A cost-effective computer-tailoring intervention would call for a broad implementation to prevent adverse neurodevelopmental effects in children due to light or moderate alcohol use. 


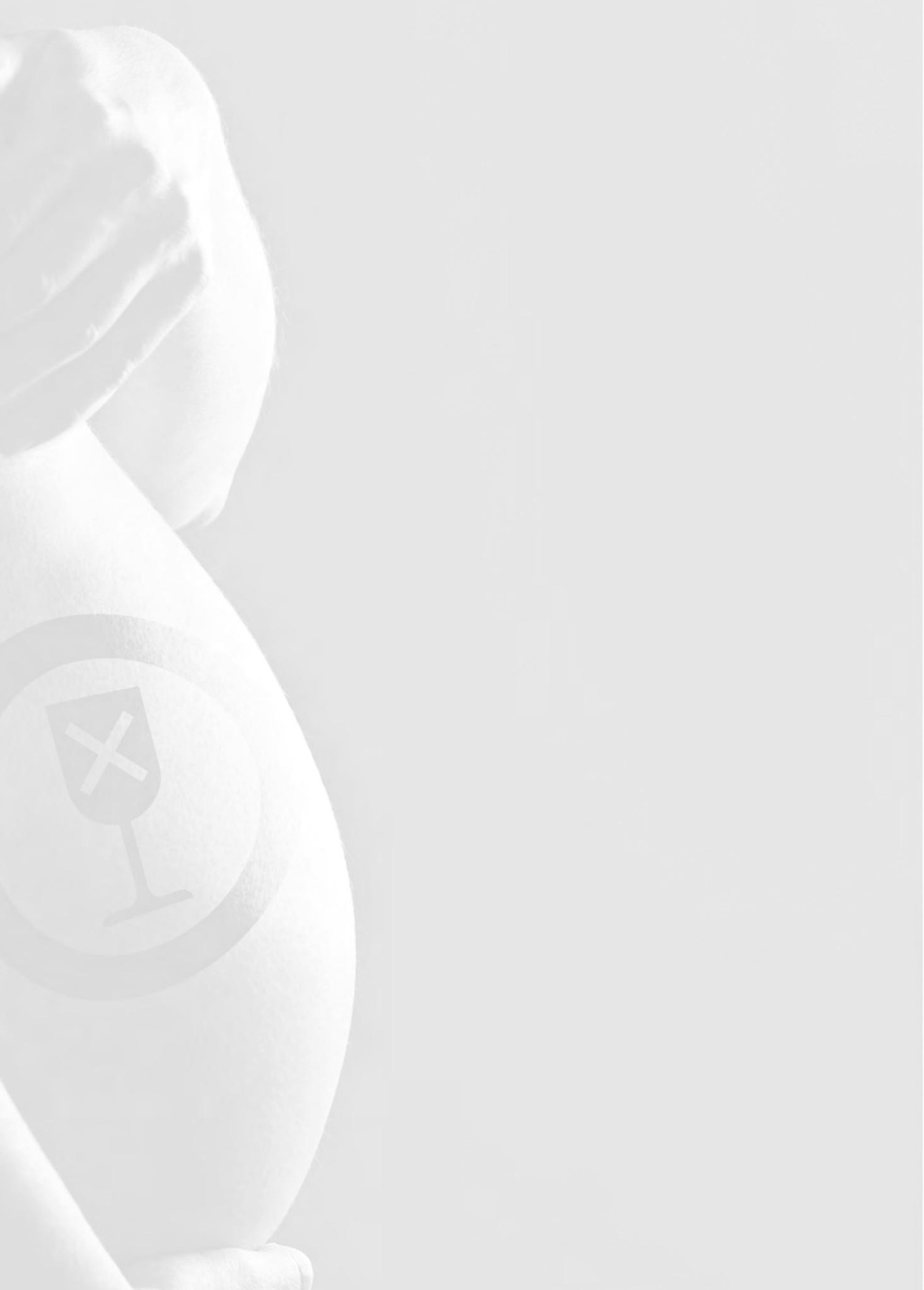




\section{CHAPTER 6}

\section{Dutch midwives' experiences with implementing health counselling to prevent prenatal alcohol use}

Published as: Van der Wulp, N.Y., Hoving, C., \& de Vries, H. (2014). Dutch midwives' experiences with implementing health counselling to prevent prenatal alcohol use. Journal of Clinical Nursing, 23(21-22): 3286-3289. 



\section{Aims}

This study aimed to explore midwives' experiences with the implementation of a brief health counselling intervention to prevent prenatal alcohol use.

\section{Background}

Low levels of prenatal alcohol exposure can cause adverse neurobehavioral effects in children (Sayal, et al., 2007). Still, around $35-50 \%$ of Dutch, pregnant women use alcohol (Health Council of the Netherlands, 2005). Brief interventions are effective in reducing prenatal alcohol use (Stade, et al., 2009), but information regarding facilitators and barriers to implementation of these interventions by health professionals such as midwives is not available. The present study investigated midwives' experiences with implementing health counselling to prevent prenatal alcohol use.

\section{Design}

Semi-structured telephone interviews were conducted with all midwives who had participated in a trial assessing the effect of a brief health counselling intervention to prevent prenatal alcohol use $(\mathrm{N}=14)$. The interview routing and the counselling protocol were based on the I-Change Model incorporating concepts which have been constructed and tested within European populations (De Vries, et al., 2006a; De Vries, et al., 2005). Ethical clearance from the Medical Ethics Committee at Maastricht University was obtained (MEC 09-3-070). The data are securely stored on a password protected computer. 


\section{Methods}

The intervention was delivered from February until December 2011. Midwives received three hour training, a manual explaining the counselling protocol and a paper intervention card to record the progress and commitments of counseled clients during three consultations. Clients were 135 pregnant women who were pregnant for a maximum of 12 weeks and had drunk alcohol since they knew to be pregnant. During the initial consultation, the first five steps of the counselling protocol were incorporated: (1) assessment of alcohol use and motivation to stop drinking, (2) perceived disadvantages of drinking and advantages of not drinking, (3) available social support and barriers for successful quitting, (4) action plans for quitting alcohol during pregnancy and (5) the identification of a quit date. Midwives were asked to advise complete alcohol abstinence during pregnancy and to provide a self-help guide. The consultation six weeks later (step 6) implied reassessing alcohol use and offering additional support for not drinking alcohol. Three months after the initial consultation, midwives discussed alcohol use and its impact on breastfeeding with their clients (step 7).

Interviews of approximately 25 minutes were conducted from April to June 2012. Midwives were interviewed about the training, the counselling intervention (Sample question 1 in Table 6.1), advantages and disadvantages of and perceived self-efficacy in prenatal alcohol use counselling (Sample question 2), the importance of the intervention (Sample question 3) and their willingness to implement the intervention in their daily practice. Eleven interviews were audio-taped and transcribed; detailed notes were taken of the three remaining interviews. Data saturation was checked and confirmed by these detailed notes.

The qualitative data analysis program MAXQDA was used to analyze the transcripts and the detailed notes. This involved identifying, coding and categorizing the primary patterns that emerged from the collected data. Categories were merged into three dominant themes: intervention application, midwives' attitude and self-efficacy in applying the intervention and the importance of the intervention and midwives' willingness to implement it. 


\section{Results}

\section{Applying the intervention at three consultations}

All midwives talked positively about the training and the manual, especially appreciating refreshing the knowledge of alcohol and pregnancy and the explanation of the protocol. Contrary to the instructions, the majority of midwives had not provided alcohol counselling at the second and third consultation, as they thought their clients did not need or like to receive this successive counselling (Citation 1 in Table 6.1). Although regarded as useful to structure the counselling sessions, some midwives had only used the intervention card after the consultation since they used digital files for their clients or did not fill in the card at all when they believed the client had already stopped drinking alcohol (Citation 2).

\section{Attitude and self-efficacy in applying the intervention}

Midwives appreciated the intervention's standardization of alcohol advice among their colleauges, the materials' simple and clear structure, and the extensiveness of the counselling (Citation 3). Disadvantages mentioned included the impracticality of implementing the paper intervention card in the otherwise digital record of the consultation and their lack of time to address the seven steps. Midwives considered the intervention too extensive when the client immediately stated that she wanted to stop using alcohol in pregnancy (Citation 4). Midwives felt capable in applying the intervention into their daily practice.

\section{Importance and implementation}

Midwives doubted the importance of the intervention as they believed that: their clients hardly used any alcohol (Citation 5); it was the client's own responsibility to decide to drink alcohol; and, prenatal alcohol was 
less important and harmful than smoking (Citation 6). Nevertheless, most midwives were willing to implement the intervention in their practice as they considered the intervention an improvement compared to their current practice (Citation 7).

\section{Conclusions}

Our interviewed midwives appreciated the clearly structured, comprehensive intervention, including the training, manual and intervention card. However, its implementation was impeded by practical issues, like the paper intervention card in a computerized consultation and lack of time, as well as doubting the importance of the intervention. Future interventions should take into account that midwives desire quick implementation of required activities, and thus need digital intervention cards when they are used to digital reports of the consultations.

Midwives' doubts about the importance of comprehensive alcohol advice in pregnancy might have led to suboptimal counselling and incorrect use of the intervention card. Hence, the importance of alcohol advice needs to be stressed in midwives' training by providing clear data on pregnant women's alcohol use and the various consequences of prenatal alcohol use versus smoking.

This study was limited to a small number of participants, as these were the only midwives who had delivered this intervention. This is the first study providing information on midwives' experiences with implementing an intervention to prevent prenatal alcohol use. It is recommended that other researchers also report implementation experiences of health professionals. Indeed, this information is necessary for the development of an intervention which can suitably be implemented in daily practice. 
Table 6.1 Examples of questions and citations of Dutch midwives concerning their experiences of implementing a brief health counselling intervention to reduce prenatal alcohol use

\begin{tabular}{ll}
\hline & Offering counselling and using intervention card at three \\
consultations
\end{tabular}

\section{Relevance to clinical practice}

Midwives may well implement a counselling intervention to prevent prenatal alcohol use, as the participating midwives considered the intervention an improvement compared to their current practice. Midwives who currently employ a digital report of their consultation will probably benefit from a compatible digital instead of a paper intervention card. Midwives need to be convinced of the importance of comprehensive alcohol advice in pregnancy, as doubts about this importance may lead to suboptimal counselling. 


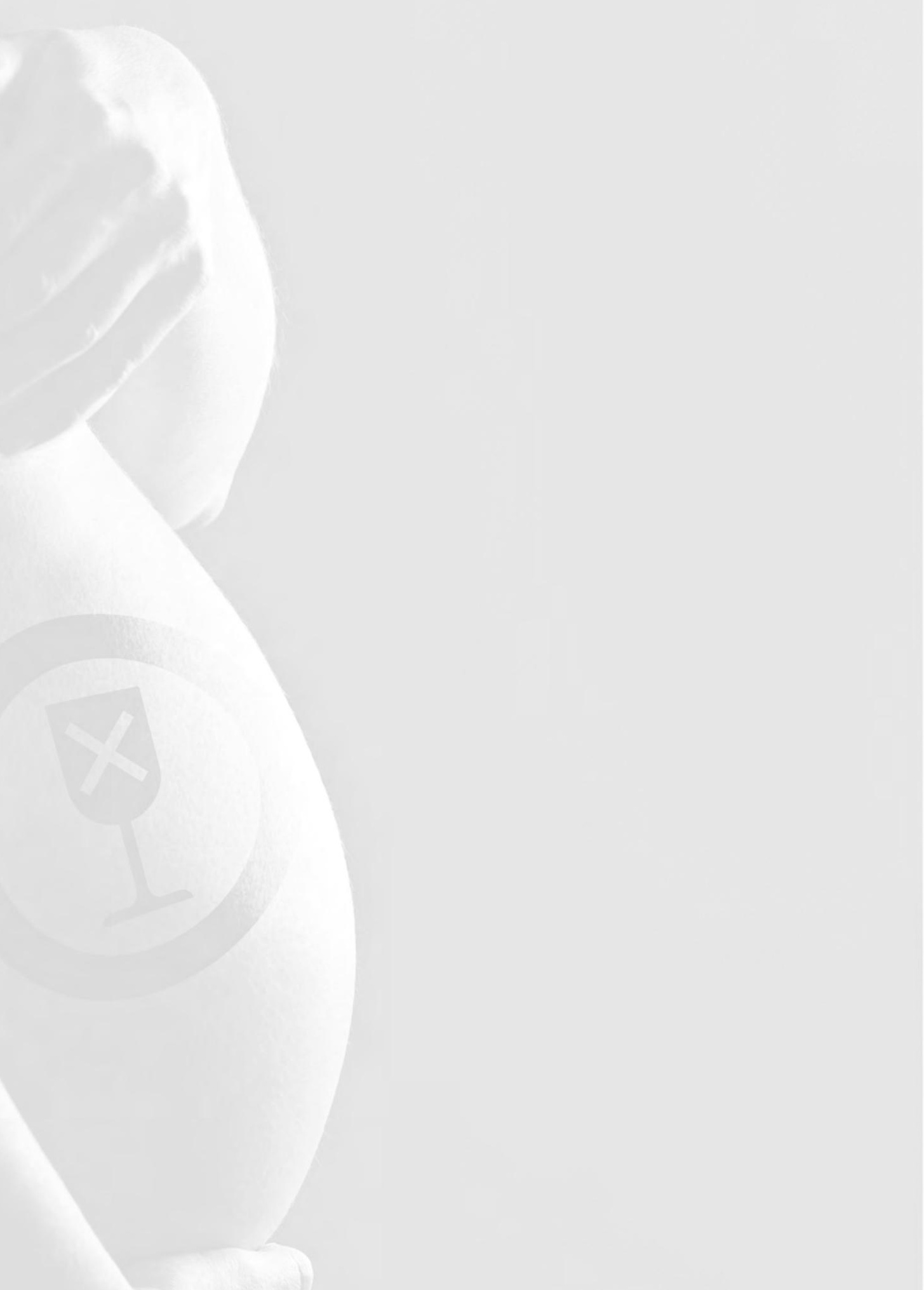


CHAPTER 7

General Discussion 



\section{Introduction}

In this dissertation, the development, effectiveness and implementation of two brief interventions aiming to reduce prenatal alcohol use were described. In doing so, the dissertation provided important information on the following topics:

1. Motivational determinants of prenatal alcohol use

2. The current state of Dutch midwives' alcohol advice

3. Effectiveness of a Health Counselling (HC) and a Computer Tailoring (CT) intervention to reduce prenatal alcohol use

4. Implementation of a $\mathrm{HC}$ intervention to reduce prenatal alcohol use

In this chapter, the main findings of each topic are described in the context of previous research, following the research questions as outlined in Chapter 1. Recommendations are formulated focusing on policy and research. In addition, methodological considerations are discussed. This chapter concludes with the presentation of five overarching, concrete recommendations to improve the reduction of prenatal alcohol use.

\section{Main findings and policy and research recommendations}

\section{Motivational determinants of prenatal alcohol use}

Research questions related to the topic of motivational determinants of prenatal alcohol use:

1. What are motivational determinants of prenatal alcohol use?

2. How do partner's norm, modelling and support as perceived by the pregnant woman and reported by her partner relate to prenatal alcohol use?

3. How are perceived and reported partner's behaviour regarding prenatal alcohol use related to each other? 
4. How important are perceived and reported partner's behaviour in explaining alcohol use of pregnant women?

5. What are determinants of partner support to abstain from prenatal alcohol use?

\section{Main findings}

The studies presented in this dissertation provided substantial new information on motivational factors (i.e. attitude, self-efficacy and social influences) of prenatal alcohol use. With regard to attitude, the results of Chapter 2 supported the findings of Peadon and colleagues (2011), showing that pregnant women using alcohol perceived fewer advantages and more disadvantages concerning alcohol abstinence during pregnancy compared to pregnant women abstaining from alcohol. There was no support for a relation between self-efficacy and prenatal alcohol use. With regard to social influences, results of Chapter 2 supported previous research (Bakhireva, et al., 2011; Chang, et al., 2011; Waterson, et al., 1990) showing a positive relation between prenatal alcohol use and partner's alcohol use ("modelling”), both as perceived by the pregnant woman and reported by her partner. Moreover, the results of Chapter 2 showed a significant relation between prenatal alcohol use and perceived and reported partner's norm. Finally, the results of this chapter showed that prenatal alcohol use was negatively related to reported partner support, whereas the relation between prenatal alcohol use and perceived partner support was not significant. Conclusively, with regard to social influences to abstain from prenatal alcohol use, the results of Chapter 2 showed that perceived partner's norm was the most important of the constructs of perceived and reported partner's influence in explaining prenatal alcohol use.

In Chapter 2, the relation between reported partner support and prenatal alcohol use was described; the determinants of this type of partner support were identified in Chapter 3. This is the first study to show that partners reporting high support to abstain from prenatal alcohol use were more likely to desire their pregnant spouse to abstain from prenatal alcohol use and to have received advice from their pregnant spouse or midwife that abstinence from prenatal alcohol use was desirable. These 
partners also had higher perceptions that the baby would experience harm from prenatal alcohol use and that harm could be more severe. Moreover, this study showed a positive relation between partner support and the partner's positive attitude on providing support, the partner's perception of having more social influences with regard to providing support, high self-efficacy and high intention to provide support during the remainder of the pregnancy.

\section{Recommendations}

The study in Chapter 2 provided important information on factors associated with prenatal alcohol use. This explorative study was crosssectional and therefore precludes statements about the causality of the relations. Some determinants of prenatal alcohol use have previously been studied in a longitudinal setting, showing firm evidence that alcohol use before pregnancy and intention to drink alcohol during pregnancy predict alcohol use during pregnancy (A. E. Anderson, et al., 2013; Skagerstrom, Chang, \& Nilsen, 2011; Zammit, et al., 2008). Thus far, it has not been investigated whether the other determinants, including awareness and motivational factors, for example partner's influence, predict prenatal alcohol use. Therefore, further longitudinal research is recommended to reveal whether knowledge and risk perception of prenatal alcohol use and motivational factors such as attitude, social influence beliefs and self-efficacy are significant in predicting prenatal alcohol use and whether their influence may differ in subgroups (e.g. depending on their motivational status or educational level)

Previous research has shown that partner involvement during pregnancy has positive effects, not only with regard to prenatal alcohol use but also in other health domains. For example, women whose partners were involved in their pregnancy were more likely to receive prenatal care early in their pregnancy and to reduce their cigarette consumption (Martin, et al., 2007). Although these findings resulted in community recommendations for greater appreciation of health care providers for the involvement of men during the prenatal period (Alio, et al., 2013), it is uncertain how this can be done effectively. The qualitative study among partners in Chapter 4 confirms that partners are suboptimally 
involved in the alcohol advice from midwives, despite the fact that they are interested in the topic and discuss it with their pregnant spouse. Partners reported a lack of attractive websites for fathers-to-be with information about their role in preventing prenatal alcohol use. Because the studies in Chapter 4 did not aim to explore how partners wished to be involved and which information they would have liked to receive through which sources and in which format, more qualitative research is needed on these issues.

Based on various theories in health behaviour (Ajzen, 2002; Bandura, 1991; De Vries, et al., 2003; Schwarzer, Lippke, \& Luszczynska, 2011), it was expected that self-efficacy of pregnant women would significantly determine prenatal alcohol use. As self-efficacy has repeatedly been shown to predict health behaviour, including smoking cessation (Hoving, Mudde, \& de Vries, 2006b; Schnoll, et al., 2011) and alcohol abstinence (Adamson, Sellman, \& Frampton, 2009), it is surprising, that this supposition was not supported by data in Chapter 2. There may be various explanations for not finding an effect.

It may be that rather than lacking self-efficacy, pregnant women lacked knowledge or reasons why they should stop drinking alcohol when pregnant. This explanation is supported by the findings described in Chapter 2 showing that pregnant women abstaining from alcohol had a significantly better knowledge about the harmfulness of prenatal alcohol use than women using alcohol. This is also supported by the results of Chapter 4 showing that pregnant women using alcohol were more often informed by their midwife that limited alcohol use would not be harmful.

It is also possible that pregnant women using alcohol were unrealistically positive about their self-efficacy in abstaining from alcohol during pregnancy, especially in social situations. Whereas the pregnant women abstaining from alcohol knew that they were capable of not drinking alcohol in difficult situations, pregnant women using alcohol had to imagine in these situations how hard it would be for them not to drink. Many people overestimate their health behaviour, for example 
regarding physical activity (Lechner, Bolman, \& Van Dijke, 2006), nutrition (Oenema \& Brug, 2003) or sun behaviour (Lechner \& de Vries, 2002); possibly some women drinking alcohol overestimated their selfefficacy regarding alcohol abstinence during pregnancy. More research, and probably more in-depth qualitative research, is needed to identify the reasons for the relatively high scores of women who drink alcohol on their self-efficacy to abstain from prenatal alcohol use.

Finally, as presented in Chapter 2, not all pregnant women and partners considered it important that pregnant women abstain from alcohol. National governments can play a role in reinforcing the alcohol abstinence norm among pregnant women and partners, for example by the introduction of health warning labels on alcoholic beverages. Such labels informing about the risks of alcohol use in pregnancy are already legally mandated in France (ICAP, 2012). The Dutch national government is currently in the process of implementing alcohol and pregnancy warnings on labels on alcoholic beverages (State Secretary for Health Welfare and Sport M.J. van Rijn, 2013). A systematic review of the experience with warning labels in the United States reported that warning labels have an effect on intentions to change drinking behaviour (Wilkinson \& Room, 2009). How the implementation of the warning labels in the Dutch context affects the general public's cognition and behaviour regarding alcohol abstinence during pregnancy could have been tested with a cohort study in which the knowledge, norm, intention and actual behaviour with regard to prenatal alcohol use of men and women are assessed before and after the implementation. Unfortunately, this is not feasible in the Dutch situation, as the implementation has already been started and the baseline assessment has not been conducted yet. In general, it is recommended that experimental studies are conducted to assess how warning label scan be used (most) effectively. In addition, experimental studies should be conducted to assess how warning labels can be used (most) effectively in general. This type of research is still scarce and has shown to result in conflicting conclusions among researchers and policy makers in the area of tobacco control (Hammond, et al., 2006; Monarrez-Espino, et al., 2014; Ruiter \& Kok, 2005). 


\section{The current state of Dutch midwives' alcohol advice}

Research questions related to the topic of Dutch midwives' alcohol advice:

6. What alcohol advice do Dutch midwives give?

7. Which difficulties do Dutch midwives experience with providing alcohol abstinence advice?

8. How do Dutch pregnant women experience midwives' alcohol advice?

9. How do partners of pregnant women experience midwives' alcohol advice?

\section{Main findings}

In Chapter 4, a qualitative study on midwives' alcohol advice was presented. This study showed that many midwives had the intention to provide alcohol abstinence advice, but mostly gave alcohol abstinence advice only to clients admitting their alcohol use. Many pregnant women were not open about their alcohol use to their midwife and midwives lacked skills to adequately screen for prenatal alcohol use. Thus, midwives might not provide alcohol abstinence advice to all pregnant women consuming alcohol. The alcohol advice of Dutch midwives also suffered from midwives' insufficient knowledge about the mechanisms and consequences of prenatal alcohol use. These lacking screening skills and insufficient knowledge about the consequences of prenatal alcohol use are shared by midwives in Sweden (Holmqvist \& Nilsen, 2010), Denmark (Kesmodel \& Kesmodel, 2011) and Australia (Jones, et al., 2011).

The difficulties mentioned by the midwives in Chapter 4 were also noted by the pregnant women receiving alcohol advice. Specifically, they were aware that midwives failed to adequately screen for prenatal alcohol use and that they lacked knowledge on the consequences of prenatal alcohol use. These experiences with midwives' alcohol advice have also been reported in other studies (Jones, et al., 2011; Kesmodel \& Schioler Kesmodel, 2002; Raymond, et al., 2009). Despite these shortcomings, pregnant women considered midwives as an important and reliable source of information on alcohol in pregnancy. This positive finding 
concerning pregnant women's experience with midwives' alcohol advice has thus far not been reported by other studies. However in a related field, it has been shown that patients perceive expert nurses and physicians as epistemic authority, meaning that they are regarded to possess valid knowledge in their specific health domain (Barnoy, Ofra, \& Bar-Tal, 2012). Pregnant women's positive evaluation of midwives' advice as presented in Chapter 4 is supported by data presented in Chapter 2 showing that pregnant women abstaining from alcohol had more often received midwife's advice to abstain from alcohol compared to pregnant women using alcohol.

Chapter 4 provided new information on partners' experience with midwives alcohol advice. This study showed that midwives did not actively involve the partner when providing alcohol advice; accordingly, partners missed being involved by the midwife. In addition, partners were dissatisfied with the limited amount of information provided by midwives and their poor prenatal alcohol consumption screening skills. Moreover, partners' view on alcohol in pregnancy was more liberal than their pregnant spouse's. And, finally, partners were actively involved in the subject of alcohol use in pregnancy as they discussed this issue with their spouses and appreciated an objective website about alcohol in pregnancy.

\section{Recommendations}

In Chapter 4, partners generally showed to have a liberal view on prenatal alcohol use, which may have a tolerating effect on their pregnant spouses' alcohol use. This suggestion is indeed supported by results of Chapter 2 showing that partners' norm is associated with prenatal alcohol use. Thus, when partners' norm is changed into a less tolerating norm, prenatal alcohol use may be reduced. Currently, there are no effective interventions available to change partners' norm regarding prenatal alcohol use. In other health behaviour research, it has been shown that personalized normative feedback can be used to change normative values, in this case regarding sun protection (Reid \& Aiken, 2013). More research is needed to find out whether personalized normative feedback given by midwives can change partners' norm 
regarding alcohol abstinence during pregnancy. For example, a module may be developed and tested which helps midwives to provide partners with personalized normative feedback to establish a change in partners' norm, so that they are convinced that alcohol abstinence during pregnancy is best. If such a module proves to be effective in changing partners' norm and subsequently effectively reduces prenatal alcohol use, midwives may be provided with an extra tool to reduce prenatal alcohol use.

The first study in Chapter 4 also showed that midwives were not confident about their alcohol consumption screening skills. Indeed, as reported in Chapters 4 and 6 and supported by previous research (Jones, et al., 2011), pregnant women under-reported their alcohol consumption to their midwife. In this dissertation, reasons for this under-reporting were not investigated. Perhaps, pregnant women are embarrassed or afraid to admit that they are drinking during pregnancy (Jacobson, et al., 2002) or they may believe that small amounts of alcohol are inconsequential (Chang, 2004). In addition, midwives' alcohol advice suffered from insufficient knowledge about the consequences of prenatal alcohol use (Chapter 4 and Jones, et al., 2011; Kesmodel \& Kesmodel, 2011). Insufficient knowledge may lead to incorrect information to clients which may not be preventive of prenatal alcohol use. Specifically, the majority of midwives interviewed for Chapter 4 wrongly believed that alcohol could not reach the embryo as long as the development of the maternal blood supply to the placenta is incomplete. Due to this information, a woman may see no reason to decrease her alcohol use in the first weeks of a future pregnancy, enhancing the chances for a miscarriage (Armstrong, McDonald, \& Sloan, 1992) and irreversible effects on growth, facial characteristics or the central nervous system (Coles, 1994). A starting point to improve midwives' alcohol advice is to increase their screening skills and improve their knowledge about the consequences of prenatal alcohol use. A Swedish national education project was successful in improving these difficulties by the use of the AUDIT (Goransson, et al., 2003) as an effective screening tool and by providing training to improve knowledge on alcohol-related health issues (Skagerstrom, et al., 2012). In order to improve the alcohol advice 
of Dutch midwives, the Dutch national government is recommended to examine the effective components of this Swedish project and explore the possibilities of adopting these elements after adjusting them to the Dutch situation.

\section{Effectiveness of a Health Counselling $(H C)$ and a Computer Tailoring (CT) intervention to reduce prenatal alcohol use}

Research questions related to the topic of the effectiveness of the $\mathrm{HC}$ and $\mathrm{CT}$ interventions to reduce prenatal alcohol use:

10. Is HC provided by midwives effective for Dutch women to stop or reduce their alcohol use in pregnancy?

11. Is CT effective for Dutch women to stop or reduce their alcohol use in pregnancy?

\section{Main findings}

Previous studies using $\mathrm{HC}$ interventions have shown that these interventions may result in increased abstinence and a reduction in prenatal alcohol consumption. However, as many uncertainties remain about the most optimal conditions of these interventions (Stade, et al., 2009), a new HC intervention was developed and tested in this dissertation. The HC intervention was based on an earlier intervention shown to be effective to reduce smoking during pregnancy (De Vries, et al., 2006a). Participating midwives received a brief manual, explaining the HC protocol and an intervention card, on which they could record the dates of the HC sessions and the clients' answers to the midwife's questions. In addition, they received three hours of training on how to provide the HC counselling. Although many participants receiving the HC intervention stopped their alcohol use, the $20 \%$ difference at three months after baseline and 18\% difference at six months after baseline with participants receiving usual care were not significant. Moreover, participants receiving the $\mathrm{HC}$ intervention did not reduce their alcohol use significantly more than participants receiving usual care. 
CT has previously proven to be effective in changing different health related behaviours (e.g. Krebs, Prochaska, \& Rossi, 2010; De Vries, et al., 2008), but has thus far not shown to effectively stop or reduce prenatal alcohol use. Therefore a new CT intervention was developed and tested in this dissertation. Respondents in the CT group received usual care from their midwife and three $\mathrm{CT}$ feedback sessions. The newly developed CT proved to be effective in stopping women's alcohol use in pregnancy. CT respondents more often stopped using alcohol (78\%) compared to UC respondents six months after baseline (55\%). Multilevel multivariate linear analyses showed that CT respondents with average or lower alcohol use before pregnancy or with average or lower social support more strongly reduced their alcohol use six months after baseline compared to UC respondents. A previously developed CT intervention aimed to reduce prenatal alcohol with one feedback session and a follow up measurement 30 days after baseline did not find a treatment effect (Tzilos, Sokol, \& Ondersma, 2011). Tzilos and colleagues suggested that the most parsimonious finding for not finding an effect may simply be due to a general lack of efficacy for this computer-delivered brief intervention. However, our study presented in Chapter 5 showed that when carefully developed, computer tailoring can be effective to stop and reduce prenatal alcohol use.

In Chapter 5, two reasons for the ineffectiveness of the HC intervention were provided. First, the non-significant effect of the present HC intervention may be explained by the lack of statistical power. The power was planned to be 0.80 , which would have been sufficient to show a significant effect with an estimated $20 \%$ difference between experimental condition and control condition. However, due to a larger intraclass correlation and a higher percentage of usual care respondents who continued drinking than we had estimated beforehand, the power turned out to be approximately 0.50. Although this amount of power was sufficient to show a significant effect of the CT intervention at six months after baseline, the $20 \%$ difference between $\mathrm{HC}$ and usual care respondents who stopped drinking alcohol three months after baseline and the $17 \%$ difference at six months after baseline were not significant. Second, the implementation of the HC intervention by 
midwives was suboptimal, as reported in Chapter 6. Also in the related field of smoking cessation in pregnancy, results were ineffective when health professionals were found to lack skills to implement their tasks as intended (De Vries, et al., 2006a; DePue, et al., 2002).

The effective CT intervention presented in Chapter 5 is a better option than $\mathrm{HC}$ as its odds ratio was larger than that of the HC condition. The studies in Chapters 5 and 6 and previous studies showed that a correct implementation of $\mathrm{HC}$ is a precondition of its effectiveness and that these conditions were not met in our study (De Vries, et al., 2006a; Hajek, et al., 2001; McLellan A, 2000; Strand, Phelan, \& Donovan, 2003). Advantageously, CT does not burden health professionals, and is thus not dependent on the quality of their counselling. Moreover, as reported in Chapters 4 and 6 and supported by previous research (Jones, et al., 2011), pregnant women are reluctant to disclose their alcohol use to health professionals which may also hamper the identification of the target group. As CT preserves a person's anonymity (Cline \& Haynes, 2001), pregnant women may be more willing to reveal their alcohol use in such intervention, which results in more relevant alcohol advice.

\section{Recommendations}

The study in Chapter 5 showed that prenatal alcohol use was not significantly reduced by the $\mathrm{HC}$ intervention. There may be various reasons why $\mathrm{HC}$ was not significant, including a suboptimal content of the protocol, poor implementation of the protocol by the midwives and lack of statistical power. It seems unlikely that the HC was not significant due to a suboptimal content of the protocol, as the protocol was based on an effective $\mathrm{HC}$ protocol on smoking cessation during pregnancy (De Vries, et al., 2006a). The other two reasons (i.e. a poor implementation of the protocol and the lack of statistical power) seem more likely. A new study in which the same protocol would be tested with enhanced circumstances may provide significant effects on prenatal alcohol use. First, these circumstances include a more extensive training of the midwives to improve the implementation of the protocol. Originally, the HC-training for the midwives consisted of two parts. The largest part of the one hour training involved the improvement of midwives' 
knowledge about the mechanisms and consequences of prenatal alcohol use. The studies in Chapter 4 showed that the midwives' alcohol advice can be improved when they learn more about the mechanisms and consequences of alcohol in pregnancy. Thus, this part of the original training appears to be essential and it is recommended to maintain this part of the training in the new study. The second part of the original training was aimed at familiarizing the midwives with implementation issues, such as how to use the HC protocol during consultations. Originally, this part took only one quarter of the time of the training. However, the study in Chapter 6 showed that the midwives had not implemented the intervention correctly, implying that this part of the training was insufficient. In the new study, it is therefore recommended that midwives are more extensively trained in how to implement the $\mathrm{HC}$ protocol and why it is important to correctly implement the protocol. A second way to enhance the circumstances is to increase the statistical power, for example by including more participants. A new study with these recommended enhanced circumstances will provide a more conclusive test for whether the developed HC protocol can be effectively used in the Netherlands to decrease prenatal alcohol use. In addition, such a study provides information which is needed (e.g. Stade, et al., 2009) to prevent future research (e.g. Wilson, et al., 2012) from wasting time and money on testing $\mathrm{HC}$ interventions not reaching a significant effect merely due to suboptimal conditions of the implementation. If a study with these enhanced circumstances provides evidence for the effectiveness of HC to decrease prenatal alcohol use, an additional study is recommended to assess the cost-effectiveness of this intervention.

Despite of the information on the influence of the partner on prenatal alcohol use collected in Chapters 2, 3 and 4, partners were not involved in the interventions tested in Chapter 5. This is due to the fact that the interventions were developed before the data of the studies in Chapters 2, 3 and 4 were analysed. The existing literature did not provide sufficient information on partner behaviour to warrant integration of this information in the interventions at that time. However, the results of the studies in Chapters 2, 3 and 4 imply that partner involvement may have an added value in the reduction of prenatal alcohol use. This 
suggestion is supported by a previously developed brief intervention to prevent prenatal alcohol use showing that the involvement of the partner enhanced the effects of the brief intervention significantly in reducing alcohol consumption (Chang, et al., 2005). At this point in time, it is difficult to indicate how partners can effectively be involved in an intervention to enhance the effect on the reduction of prenatal alcohol use. A Cochrane review on the increase of partner support to enhance smoking cessation could not find evidence that the interventions actually increased partner support (Park, Tudiver, \& Campbell, 2012). Consequently, the interventions intended to increase partner support did not result in an enhancement of long-term quit rates compared to smoking cessation programmes without a partner support component. A two-step approach is recommended to analyse whether the increase of partner support actually leads to enhanced effects on the reduction of prenatal alcohol use. First, an experimental study could be designed to test how partner support can be increased, for example by encouraging midwives to direct their alcohol advice not only to the pregnant woman but also to the partner as suggested in Chapter 2 . Second, when this experimental study results in a successful manipulation of increasing partner support, a randomized controlled trial can be used to test whether the manipulation enhances the effect on the reduction of prenatal alcohol use.

The CT intervention presented in Chapter 5, should not only be effective in stopping prenatal alcohol use, but should also be cost effective (Ahern, Kreslake, \& Phalen, 2006). A recent study estimating the costeffectiveness of competing health care systems in curbing alcohol use at the national level showed that the introduction of new eHealth technologies would substantially increase the cost-effectiveness of the Dutch health care system for alcohol use disorders: every euro spent under eHealth technologies generates $€ 1.62$ in health-related value (F. Smit, et al., 2011). This finding is replicated by other recent studies with regard to smoking cessation (E. S. Smit, et al., 2013b) and lifestyle intervention (Schulz, et al., 2014), provided that effective health behaviour models are appropriately used in these eHealth technologies. Yet, no studies have been conducted regarding the cost-effectiveness and cost-utility of an Internet-based intervention aimed at the reduction 
of prenatal alcohol use. Therefore, research is recommended to assess the cost-effectiveness of the $\mathrm{CT}$ intervention.

If future research shows that the $\mathrm{CT}$ intervention is not only effective in stopping prenatal alcohol use but is also cost effective, it is recommended to implement the program nationwide. This would imply that this intervention is made well accessible for pregnant women using alcohol. Web-based self-help interventions are appealing to problem drinkers (Cunningham, Humphreys, \& Koski-Jannes, 2000; Koski-Jannes, et al., 2007) and they fit well in an era in which self-help is becoming an essential component of our health care system (Riper, et al., 2009). Furthermore, research has shown that the implementation of the Dutch e-health intervention "Drinking Less" aimed at reducing adult problem drinking has shown to be feasible, well-accepted, and effective for curbing adult problem drinking in the community (Riper, et al., 2009). Although Drinking Less aims at a different target group with a slightly different behavioural outcome (i.e. drinking less rather than complete alcohol abstinence during pregnancy), the implementation of the CT intervention may benefit from lessons learned from implementing Drinking Less. Specifically, the implementation of Drinking Less profited from promotion in advertisements in national newspapers and on alcohol- and health-related websites where people who were interested to moderate their alcohol use were invited to visit the website of Drinking Less. In addition, research has shown that usage of eHealth interventions can be further improved, for example by email and mobile phone prompts (Cremers, et al., 2014). Thus, an additional study on the potential facilitating and hindering factors for the use of the $\mathrm{CT}$ intervention may provide valuable information to optimize the nationwide implementation of the program.

To reduce prenatal alcohol use, the studies in this dissertation focused on the strategies of $\mathrm{HC}$ and $\mathrm{CT}$, which can be classified as informational indicated prevention (Foxcroft, 2013). This implies that individuals who screen positive for a behavioural risk factor (in this case, pregnant women using alcohol) receive normative feedback aimed at intervening with this risky behaviour. The largest disadvantage of such strategies is that they 
concern secondary prevention (Johnson, et al., 2010): pregnant women are already using alcohol, while greater effect is obtained when women stop using alcohol before they are pregnant. Moreover, as mentioned in Chapter 2 and previous research (Chang, et al., 2007), alcohol use before pregnancy significantly determines alcohol use in pregnancy. Thus, another strategy would be to reduce alcohol use in general. For this purpose, environmental universal interventions (Foxcroft, 2013) have shown to be effective, aiming to limit maladaptive behaviours of the general public. Research on alcohol policy has provided evidence for the effectiveness of three environmental universal interventions (Alcohol Public Policy Group, 2010; P. Anderson, Chisholm, \& Fuhr, 2009), that is (1) increasing alcohol prices (P. Anderson, et al., 2009; Wagenaar, Salois, \& Komro, 2009; World Health Organization, 2007), (2) restricting availability of alcohol, for example by reducing hours and days of sale, limiting the number of alcohol outlets and restricting retail access to alcohol (Gruenewald, 2007; Livingston, Chikritzhs, \& Room, 2007; Room, 2002), and (3) banning alcohol marketing (Casswell, 2004; Koordeman, Anschutz, \& Engels, 2012; Snyder, et al., 2006). As these interventions have shown to be highly cost-effective strategies to reduce alcohol related harm (P. Anderson, et al., 2009) and in order to make an actual difference in the reduction of prenatal alcohol use, the Dutch government is recommended to make alcohol more expensive and less available and to ban alcohol advertising.

\section{Implementation of a HC intervention to reduce prenatal alcohol use}

Research question related to the topic of the implementation of a $\mathrm{HC}$ intervention to reduce prenatal alcohol use:

12. What are midwives' experiences with the implementation of a brief health counselling intervention to prevent prenatal alcohol use? 


\section{Main findings}

Information on implementation issues is needed to ascertain that health professionals are capable of putting a $\mathrm{HC}$ intervention into practice and that necessary conditions for a widespread implementation of the HC intervention are provided (Jago \& Sebire, 2012). As previously published randomized trials testing the efficacy of brief interventions to reduce prenatal alcohol did not provide any information on implementation issues (Stade, et al., 2009), the study presented in Chapter 6 is the first to show that health professionals invited to implement a brief intervention to reduce prenatal alcohol use are impeded by several implementation issues.

Although, midwives appreciated the clearly structured, comprehensive intervention to prevent prenatal alcohol use, the implementation was impeded by practical issues similar to the studies in the field of smoking cessation in pregnancy (e.g. De Vries, et al., 2006a; Hajek, et al., 2001; Strand, et al., 2003), like lack of time and the use of a paper intervention card in a computerized consultation. Even though most midwives considered the intervention an improvement compared to their current practice, the findings presented in Chapter 6 stress that in developing a brief intervention applied by midwives, it is necessary to minimize and facilitate the activities as midwives experience an excessive workload (Wiegers, 2007). In Chapter 6, it was also shown that the implementation was impeded by midwives' doubts about the importance of the intervention. The underestimation of the importance of offering comprehensive alcohol advice in pregnancy may be due to midwives' tendency to underrate the number of pregnant women using alcohol (e.g. Chapter 4) and their insufficient knowledge on the consequences of prenatal alcohol use (see Chapter 4 and Holmqvist \& Nilsen, 2010; Kesmodel \& Kesmodel, 2011). Health professionals' notion of the importance of alcohol advice may be improved when they receive additional education, for instance regarding the number of pregnant women using alcohol and the consequences of prenatal alcohol use for the unborn baby. 
The study presented in Chapter 6 showed that midwives had not implemented the intervention as intended. They had only provided counselling at the initial consultation, not at the second and third consultation, because they thought their clients did not need it or they were worried that their clients did not like to receive alcohol counselling several times. Moreover, they had not used the intervention card as it was intended. Some midwives had filled in the intervention card after the consultation, as it did not fit in the otherwise digital file of the client; and some midwives had not filled in the intervention card at all, if they believed the client had already stopped drinking alcohol. A more adequate implementation may be realized when the health care providers are trained more extensively with for example a stronger focus on the importance of providing alcohol advice as shown by O'Conner and Whaley (2007). Alternatively, a more adequate implementation may be expected when trained health counsellors are employed for this task, as shown by Chang and colleagues (1999) and Reynolds and colleagues (1995). However, a disadvantage of using trained health counsellors under optimal conditions may be that the external validity becomes at risk, as it is uncertain whether these interventions are still effective when implemented in routine care.

\section{Recommendations}

The study in Chapter 6 has shown that the evaluation of health professionals' experiences with the implementation of HC interventions can help to understand why an intervention is or is not effective. Future HC studies are therefore recommended to collect data on implementation issues of health professionals.

Relatively few process evaluation studies assessing health professionals' experiences with the implementation of interventions have been published thus far (Jago \& Sebire, 2012). This may not only be attributable to the fact that researchers do not always collect process evaluation data, but also because of the difficulties with publishing process evaluations (Jago \& Sebire, 2012). Many of these studies are small with simple designs that do not have random assignment of participants or rely heavily on qualitative methods, both of which also hold for the study 
in Chapter 6. According to Jago and Sebire (2012), reviewers may raise issues of the representativeness of the sample and therefore reject the papers. However, when such studies are not published, many research groups may unknowingly replicate comparable process evaluation studies, unnecessarily increasing the costs of research (Jago \& Sebire, 2012; Moore, et al., 2014). Fortunately, as shown in Chapter 6, the Journal of Clinical Nursing does accept (short) papers on process evaluations on the implementation of interventions for publication. In order to share process evaluation information among researchers, it would be recommendable if more journals would adopt a similar publication strategy.

\section{Methodological considerations: Strengths and weaknesses}

Most strengths and weaknesses of the studies have already been discussed in the previous chapters. This section provides an overview of the main strengths and weaknesses of the dissertation.

One strength of the studies in this dissertation is the fact that the subject of this dissertation has been investigated from different perspectives. The studies in Chapters 4 and 6 investigated the midwives' perspective on the prevention of prenatal alcohol use. Studies in Chapters 2, 3 and 4 investigated the perspective of the partner of the pregnant woman. Finally, the pregnant women's perspective was investigated in Chapters 2, 4 and 5. By involving these perspectives, the relevant stakeholders regarding this topic are included. Although also obstetricians provide alcohol advice to pregnant women, the focus on midwives in this dissertation is justified by the fact that pregnant women more often receive alcohol advice from midwives than from obstetricians (Wiegers, 2007). Thus, the midwives most strongly represent the perspective of health providers with regard to the prevention of prenatal alcohol use.

Another strength of this dissertation was the effect assessment of the interventions in routine care. The use of an effectiveness study, in which the effects of an intervention are studied in routine care, increases the 
external validity of the intervention (Singal, Higgins, \& Waljee, 2014). This is also previously done by O'Connor and Whaley (2007), who relied on usual care providers to provide the prenatal alcohol counselling. Alternatively, the external validity of $\mathrm{HC}$ interventions is at risk with efficacy studies. Two previously developed health counselling interventions (Chang, et al., 1999; Reynolds, et al., 1995) proved to be effective with trained health counsellors under optimal conditions. It is uncertain whether these interventions are still effective when implemented in routine care.

Besides the reported strengths, the studies were also subject to limitations, which should be considered when interpreting the results of this dissertation.

One limitation is that all of the studies relied on self-reported outcome measures. This included self-reported prenatal alcohol use in Chapters 2 and 5 and self-reported partner support in Chapter 3 (as well as selfreport in the qualitative studies in Chapters 4 and 6). This use of selfreport might have caused bias, for example due to social desirable answers (Adams, et al., 1999). Pregnant women may have underrated their alcohol use and partners may have overrated their level of support. We tried to limit social desirable answers by stressing the confidentiality of their response and by stating that there were no 'wrong answers'. In order to prevent pregnant women to deny their alcohol use when they believed that the amount was negligible, we stressed that we were even interested in the use of one single sip of alcohol the woman had consumed since she knew she was pregnant. The Quantity-FrequencyVariability questionnaire was used to assess the prenatal alcohol use of the pregnant women as this questionnaire has previously shown to provide relatively reliable alcohol reports (Dotinga, et al., 2006) and has a high validity (Lemmens, Tan, \& Knibbe, 1992). Nevertheless, underreport may still be possible as previous research has shown that the prevalence of prenatal alcohol exposure is on average four times higher when using meconium testing compared to the prevalence estimates obtained using maternal self-reports (Lange, et al., 2014). However, meconium testing is not relevant in the context of this dissertation as 
it cannot determine alcohol exposure in the first trimester and can only be done after the baby is born. Another direct marker of alcohol use is phosphatidylethanol (PEth) in blood (Viel, et al., 2012). The so-called PEth-assay can be conducted on maternal blood at each moment during pregnancy. This new test is promising for early detection of alcohol use during pregnancy and is for that purpose currently piloted in the Sophia hospital in Rotterdam. If this pilot test provides support for the usefulness of the PEth-assay to detect prenatal alcohol use and indicates that pregnant women are willing to participate in such a measure which is potentially burdensome, researchers may consider using PEth-assay as an additional, objective measure to assess alcohol use in pregnancy. Finally, alternatives to the self-reports of partner support could have been considered, such as observational and daily diary methods (Gottlieb \& Bergen, 2010). Cutrona and Suhr (1994) have developed a coding scheme for capturing support transactions between spouses which were derived from couples' descriptions of the support they wanted to receive from their partners upon the occurrence of stressful events. Moreover, Dehle and colleagues (2001) conducted a daily diary study in which married partners completed the Support in Intimate Relationships Rating Scale (SIRRS; Cutrona \& Suhr, 1992) on seven consecutive evenings. Cutrona's coding scheme or the SIRRS could be adjusted to capture the support pregnant women want to receive and partners provide in relation to alcohol abstinence during pregnancy. The match or mismatch between these two constructs could be tracked with a daily diary study and used as outcome variable to analyse the correlates of partner support to abstain from prenatal alcohol use.

However, due to the brevity and convenience, self-report measures of partner support are most widely used (Gottlieb \& Bergen, 2010) and the self-report measure used in Chapter 4 is consequently the most advantageous method.

Another limitation is that, although the CT intervention has shown to be effective, pregnant women's experiences with this intervention have not been evaluated yet. Moreover, in the CT condition a relatively large number of participants dropped out and could not be reached to 
find out why they dropped out. This drop out may have been related to the intervention ("intervention drop out"; Sohanpal, et al., 2012), for example participants got annoyed by the intervention or considered the intervention unnecessary as they had stopped drinking alcohol anyway. Alternatively, there may have been other reasons as well, as for example a miscarriage, annoyance with participating in a study, or a change of email address ("study drop out"; Sohanpal, et al., 2012). It is important to understand if the CT-drop out was related to the intervention, because that would imply that the missing at random (MAR) assumption applied in the analyses of the effect study would have been erroneous. A content analysis on the emails sent by the $\mathrm{CT}$ participants to the researchers and interviews with CT participants who did and who did not complete the intervention may shed more light on this issue. Furthermore, as process evaluation questions were included in the final effect study questionnaire, analysis of these answers may provide valuable insight into the pregnant women's opinions on the $\mathrm{CT}$ intervention.

\section{General Recommendations}

The discussion of the studies in this dissertation leads to the following overarching, concrete recommendations to further improve the reduction of prenatal alcohol use.

$\triangleright$ Pregnant women are recommended to use the CT intervention. When pregnant women are participating in the CT intervention for a period of six months, a reduction of prenatal alcohol use may be expected.

$\triangleright$ Midwives are recommended to explore ways to improve their alcohol advice. If a quantitative analysis among a representative sample of midwives supports the findings of Chapter 4, midwives' alcohol advice can be enhanced when they improve their alcohol screening skills, they increase their knowledge on the mechanisms and consequences of prenatal alcohol use and they involve partners in their alcohol advice. 
$\triangleright$ Partners are recommended to strengthen their alcohol abstinence norm as a partner's alcohol abstinence norm is related to alcohol abstinence of his pregnant spouse. Partners may seek active involvement in prenatal alcohol advice by asking midwives or search for prenatal health information on the internet. In addition, public health communication strategies may be needed to convince partners of a complete alcohol abstinence norm.

$\triangleright$ Researchers are recommended to test the HC protocol under enhanced circumstances, in order to develop an effective HC protocol for midwives who want to help their clients to reduce their alcohol use. Enhanced circumstances include a better training of the midwives to improve their implementation and more participants per practice to increase statistical power.

$\triangleright$ Because alcohol use during pregnancy is related to alcohol use before pregnancy, the Dutch national government is recommended to implement cost-effective environmental universal interventions to reduce alcohol use in general. These interventions encompass an increase in the price of alcohol, a restriction of the availability of alcohol and banning alcohol marketing. 



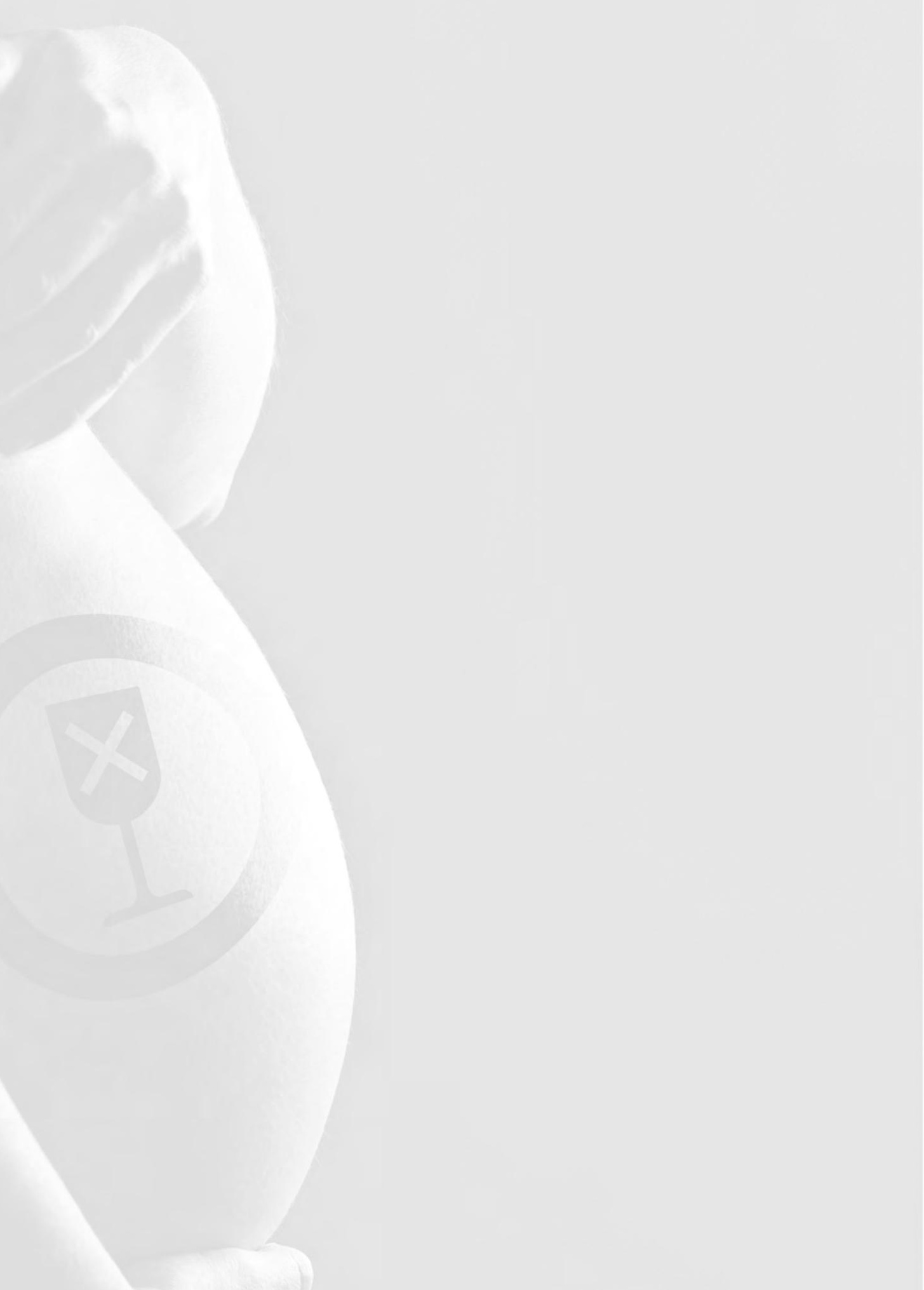




\section{Valorisation Addendum}





\section{Relevance}

The relevance of this research on the development, evaluation and implementation of two brief interventions to prevent prenatal alcohol use emanates from the harmful effects of prenatal alcohol exposure in combination with the high number of Dutch pregnant women using alcohol. As described in Chapter 1, alcohol use in pregnancy is risky for the unborn baby. Alcohol in the foetus affects various cellular processes that occur during the foetal development (e.g. van Faassen \& Niemela, 2011). This can lead to a variety of consequences, such as miscarriage, deformation of body parts, facial abnormalities and neurological damage (Bertrand, et al., 2004). The severity of the damage is dose dependent which implies that the more alcohol the mother drinks during pregnancy, the more severe the harm to the unborn baby (Patra, et al., 2011). In this dissertation, it is estimated that 2 to $6 \%$ of all Dutch children suffer from the consequences of prenatal alcohol use.

Besides of the risk of prenatal alcohol use, the literature review in Chapter 1 also showed a high prevalence of prenatal alcohol use. Based on various recent studies, it is suggested that approximately 30\% of the Dutch pregnant women use alcohol. This approximate estimation of Dutch pregnant women using alcohol in combination with the estimation that 2 to $6 \%$ of the Dutch children suffer from the consequences of prenatal alcohol use signifies the relevance of this research on the reduction of prenatal alcohol in pregnancy with regard to public health.

Because prenatal alcohol use has health-related consequences for children who are prenatally exposed to alcohol, it consequently puts an economic burden on society (Popova, et al., 2013). Direct health-care costs related to prenatal alcohol use include acute care, psychiatric care, day surgery, and emergency department services; indirect costs concern productivity loss due to morbidity. Society pays for the costs of prenatal alcohol use because of higher health insurance, medical related costs and higher prices for health-care products and services. Thus, by aiming to reduce prenatal alcohol use, this research has health-related and consequently economic relevance for society. 


\section{Target groups}

Various target groups profit from this research. Clearly, pregnant women profit from the research findings presented in Chapter 5, which encompass a newly developed, effective intervention to reduce prenatal alcohol use. Chapter 4 showed that pregnant women may feel reluctant to disclose their actual alcohol use to their midwife and consequently they do not always receive accurate alcohol advice. Chapter 5 suggested that these women may feel more confident to open up to the anonymized computer tailored intervention. Thus, pregnant women may now receive accurate and reliable information from the intervention on alcohol and pregnancy that matches their actual alcohol use, besides of the information they already receive from their midwives.

Another target group profiting from this research comprises partners of pregnant women. The research findings on the motivational determinants of prenatal alcohol use have shown that partners matter in various ways when it comes down to alcohol use in pregnancy. Despite the fact that unhealthy behaviour of their pregnant spouse has negative consequences for their child, partners have no legal authority over this behaviour or generally over their child before birth. Nevertheless, this research shows that the partners' alcohol use, their norms on prenatal alcohol use and their support to abstain from prenatal alcohol use are related to their pregnant spouse's behaviour. The present research lays the foundation for further research into the causality of this relationship, which may find that partners have unobjectionable means to influence their pregnant spouse's behaviour and protect their child's health.

Partners of pregnant women also profit from the research findings on Dutch midwives' alcohol advice. The present research showed that Dutch midwives did not actively involve the partner when providing alcohol advice, while partners missed being involved by the midwife. The results of this research have been discussed with staff of the Royal Dutch Organization of Midwives (KNOV), who have been recommended to encourage their members to involve partners when providing alcohol advice. 
Another target group profiting from this research comprises midwives. This dissertation has provided insight into the current practice of Dutch midwives with regard to their alcohol advice. The Dutch national newspaper Trouw published an article on these findings (Den Blijker, 2010). In addition, Parliamentary Questions were asked (Tweede Kamer, 2010), encouraging the responsible Minister for Health, Welfare and Sport and the Minister for Youth and Families to take appropriate actions to improve midwives' alcohol advice. Finally, these research findings have been discussed with the staff of the KNOV, who have been recommended to organize training for midwives to increase their knowledge about mechanisms and consequences of prenatal alcohol use.

The research findings of all topics have been published on the website alcoholenzwangerschap.nl. This website with scientifically based information on alcohol use during pregnancy has sections for pregnant women in the preconception, conception and breastfeeding phase and for professionals. Approximately 10.000 people per month visit this website, which indicates that this website meets a particular demand for alcohol and pregnancy-related information.

The research findings are also of interest for the Dutch College of General Practitioners (GPs) which supports the use of e-mental health in GP care. The GP setting can be considered an important access point to reach pregnant women. Indeed, a quality label for e-mental health interventions in GP care has invited the Dutch Institute of Alcohol Policy STAP to submit the intervention in this dissertation for an evaluation. This quality label called Online Hulpstempel is meant to warrant the quality of e-mental health in GP care. According to the invitation for this evaluation, this intervention was selected because it was mentioned in the GP guidelines or it was nominated by professionals working in the GP setting. Apparently, this intervention meets a demand for effective e-health interventions that can be used in the GP setting. 
Also health educators and policy makers profit from this research. For example, the research on the determinants of prenatal alcohol use presented in Chapter 2 showed that specifically older and welleducated pregnant women continue their alcohol use in pregnancy. The specification of the women who use alcohol during pregnancy helps health educators and policy makers to tune their actions to prevent prenatal alcohol use to the characteristics of these women. For example, the Dutch Institute for Alcohol Policy STAP and the Trimbos Institute have jointly developed a brochure for pregnant women on alcohol, tobacco and drug use. The section on tobacco use during pregnancy targeted women with a low social economic status (Phares, et al., 2004), whereas the section on alcohol use during pregnancy targeted women with a high social economic status. Moreover, politicians can now formulate correct Parliamentary questions prompting the responsible Minister and Secretary of State for Health, Welfare and Sport to take targeted activities for well-educated pregnant women to prevent prenatal alcohol use (Tweede Kamer, 2012, 2015a). In turn, the Minister and Secretary of State used this research for their answers to the Parliamentary Questions (Tweede Kamer, 2010, 2015b).

Policy makers of the Dutch Ministry of Health, Welfare and Sport might also have been interested in the results of this research to improve Dutch natal care. Since 2008, there is a lot of attention for the perinatal mortality in the Netherlands (Stuurgroep Zwangerschap en Geboorte, 2009). This is due to an international publication showing relatively high perinatal mortality in the Netherlands compared to other European countries (Peristat II, 2008). Dutch government consequently asked an external steering committee to bring an advice on optimizing the care during pregnancy and birth, in order to reduce, wherever possible, the perinatal mortality and morbidity. In their advice, this committee stressed the necessity to provide clear and unambiguous information on lifestyle factors to support a pregnant woman to carry out her pregnancy as healthy and safe as possible (Stuurgroep Zwangerschap en Geboorte, 2009). The research presented in this dissertation might guide policy makers who need to realize this advice with respect to the lifestyle factor alcohol use during pregnancy. 
Also intervention developers profit from this research. Research on implementation issues as provided in Chapter 6 is scarce, although knowledge of the experiences of midwives with the implementation of brief interventions provide valuable information for developers of future interventions. With these research findings, developers are better equipped to match future interventions with midwives' practice.

This research may also have been beneficial for children in general. Beyond the publication in scientific articles, the research findings have received societal attention in national newspapers, on radio and television, on websites and in three sets of Parliamentary questions. This research may have led directly and via the societal attention also indirectly to the reduction of prenatal alcohol use. However, more research is needed to confirm that this has consequently resulted in a decrease of children experiencing harm caused by alcohol exposure during pregnancy.

\section{Product}

The research findings of Chapter 5 encompassed the newly developed computer tailoring intervention which has shown to be effective in reducing prenatal alcohol use. This online self-help program is intended for pregnant women who use alcohol. By using the intervention, participants learn more about the effects of alcohol consumption during pregnancy. They also get advice of how to reduce or quit using alcohol. This intervention is offered through the Internet and is accessible via a chosen username and password. It can therefore be used anywhere and anytime, provided there is access to a computer, tablet or smartphone with internet access.

At the beginning of participating this intervention, the participant completes the first questionnaire, which is immediately followed by the first feedback letter. Six weeks after the first session, the participant receives an email asking her to re-log on to the website (followed by up 
to two reminders after respectively two and four weeks). This second session consists of a short questionnaire, which is followed by a second feedback letter. Three months after the first session, the participant receives an email asking her to re-log on to the website (again followed by up to two reminders after two and four weeks). The third session consists of a more extensive questionnaire, which is followed by a third feedback letter.

In the feedback letters, pregnant women receive personalised feedback on their drinking behaviour, their attitude, perceived social influence, self-efficacy and how to prepare to quit. The intervention is effective and suitable for all pregnant women using alcohol, independent of their motivation and intention to quit.

\section{Innovation}

There are several internet interventions available in the Netherlands to reduce problematic alcohol use, such as Drinktest.nl (Boon, et al., 2011) and Minderdrinken.nl (Riper, et al., 2008), but none of these interventions are designed for pregnant women. As also mentioned in Chapter 5, to our knowledge only one computer-tailored intervention for alcohol use in pregnancy has previously been developed. This intervention developed by Tzilos and colleagues (2011) was not found to effectively reduce prenatal alcohol use compared to an assessment-only condition. Chapter 5 showed that the intervention developed in this dissertation was effective and it may therefore be world's first effective internet intervention reducing prenatal alcohol use.

\section{Implementation}

With a follow-up research proposal, the Dutch Institute for Alcohol Policy obtained a grant from the Netherlands Organisation for Health Research and Development (ZonMW) to implement the intervention developed in this dissertation. The implementation project of the intervention which 
was now called Zero for Nine Months (Negen Maanden Niet; 9MN in Dutch) took place in 2014. The project focused on two target groups. First, it focused on pregnant women by increasing their awareness of the existence of the intervention and by making efforts for them to warrant a free use of the intervention also in the future. Second, it focused on health professionals by increasing their awareness of the existence of the intervention and by encouraging them to refer their clients to this intervention.

With regard to the focus on pregnant women, the following actions were taken. The feedback of the intervention was adjusted, by omitting the references to the research described in Chapter 5. The intervention was incorporated in the website www.alcoholenzwangerschap.nl, with approximately 10.000 visitors per month, a well visited website. Meetings were arranged with health insurances to discuss the possibility of funding in order to warrant a free use of 9MN also in the future.

With regard to the focus on health professionals, the following actions were taken. The professional associations of midwives, gynaecologists and general practitioners were contacted to discuss the possibility to include a reference to $9 \mathrm{MN}$ in their associations' guidelines. References to the 9MN website were applied to flyers and brochures that midwives and other health care professionals spread among their clients. The intervention was presented at meetings for gynaecologists and midwives at various locations in the Netherlands. Publicity was gained by link exchanges with relevant governmental websites. Developers of software programs were contacted to incorporate references to 9MN in their software.

The project has achieved the following results. First, 9MN is now publicly available on www.negenmaandenniet.nl and is being supported by the Dutch Institute for Alcohol Policy STAP. Pregnant women can use this intervention freely. Second, pregnant women are made aware of the intervention by extensive communication on www.alcoholenzwangerschap.nl and because a reference to $9 \mathrm{MN}$ has been applied to the flyers and brochures that are distributed by health 
professionals. Third, many health professionals have visited one of the 50 presentations that were given throughout the country. Moreover, they have been notified of $9 \mathrm{MN}$ through the communication with the professional associations of midwives, gynaecologists and general practitioners. Finally, a meeting with people from Achmea, the largest health insurance company in the Netherlands, resulted in the inclusion of $9 \mathrm{MN}$ in the health care purchasing contracts of the health insurance company with midwifery practices.

Although the implementation project has ended at the end of 2014, the Dutch Institute of Alcohol Policy STAP is still working on increasing the use of the intervention. Specifically, efforts are being taken to improve the layout of the website in order to increase the attractiveness of the intervention. Moreover, STAP is trying to achieve the incorporation of a reference to the website of $9 \mathrm{MN}$ in the software used by health professionals.

\section{Conclusion}

To conclude, the research findings presented in this dissertation have social relevance and a societal value for a variety of target groups. This research has resulted in an innovative product which helps pregnant women to reduce their alcohol use. This product can now freely be used by pregnant women using alcohol anywhere and anytime, provided there is access to a computer, tablet or smartphone with internet access. This product is valuable for health professionals who can refer their clients to the intervention website, providing them an effective tool to prevent prenatal alcohol use. Altogether, this research has contributed to a reduction of prenatal alcohol use, which has hopefully resulted in a reduction of the number of children experiencing harm caused by alcohol exposure during pregnancy. 
References 
A

Adams, G., Gulliford, M.C., Ukoumunne, O.C., Eldridge, S., Chinn, S., \& Campbell, M.J. (2004). Patterns of intra-cluster correlation from primary care research to inform study design and analysis. Journal of Clinical Epidemiology, 57(8):785-794.

Adams, A., Soumerai, S., Lomas, J., \& Ross-Degnan, D. (1999). Evidence of self-report bias in assessing adherence to guidelines. International Journal for Quality in Health Care, 11(3), 187-192.

Adamson, S. J., Sellman, J. D., \& Frampton, C. M. A. (2009). Patient predictors of alcohol treatment outcome: A systematic review. Journal of Substance Abuse Treatment, 36(1), 75-86.

Ahern, D. K., Kreslake, J. M., \& Phalen, J. M. (2006). What is eHealth (6): perspectives on the evolution of eHealth research. Journal of Medical Internet Research, 8(1).

Ajzen, I. (1985). From intentions to actions: A theory of planned behavior. In J. Kuhl, \& Beckmann, J. (Ed.), Action Control: From cognition to behavior (pp. 11-39). Berlin, Heidelberg, New York, Tokyo: Springer-Verlag.

Ajzen, I. (2002). Perceived behavioral control, self-efficacy, locus of control, and the theory of planned behavior. Journal of Applied Social Psychology, 32(4), 665-683.

Alcohol Public Policy Group (2010). Alcohol: No Ordinary Commodity - a summary of the second edition. Addiction, 105(5), 769-779.

Alio, A. P., Lewis, C. A., Scarborough, K., Harris, K., \& Fiscella, K. (2013). A community perspective on the role of fathers during pregnancy: a qualitative study. BMC Pregnancy Childbirth, 13, 60.

Alvik, A., Heyerdahl, S., Haldorsen, T., \& Lindemann, R. (2006). Alcohol use before and during pregnancy: a population-based study. Acta Obstetricia et Gynecologica Scandinavica, 85(11):1292-1298.

American Academy of Pediatrics Committee on Substance Abuse (1993). American Academy of Pediatrics Committee on Substance Abuse and Committee on Children with Disabilities: Fetal alcohol syndrome and fetal alcohol effects. Pediatrics, 91(5), 1004-1006.

Anderson, A. E., Hure, A. J., Forder, P., Powers, J. R., Kay-Lambkin, F. J., \& Loxton, D. J. (2013). Predictors of antenatal alcohol use among Australian women: a prospective cohort study. BJOG, 120(11), 1366-1374.

Anderson, P., Chisholm, D., \& Fuhr, D. C. (2009). Effectiveness and cost-effectiveness of policies and programmes to reduce the harm caused by alcohol. Lancet, 373(9682), 2234-2246.

Armstrong, B. G., McDonald, A. D., \& Sloan, M. (1992). Cigarette, alcohol, and coffee consumption and spontaneous abortion. American Journal of Public Health, 82(1), 85-87.

\section{B}

Bakhireva, L. N., Wilsnack, S. C., Kristjanson, A., Yevtushok, L., Onishenko, S., Wertelecki, W., et al. (2011). Paternal drinking, intimate relationship quality, and alcohol consumption in pregnant Ukrainian women. Journal of Studies on Alcohol and Drugs, 72(4), 536-544.

Bakker, R., Pluimgraaff, L. E., Steegers, E. A., Raat, H., Tiemeier, H., Hofman, A., et al. (2010). Associations of light and moderate maternal alcohol consumption with fetal growth characteristics in different periods of pregnancy: The Generation R Study. International Journal of Epidemiology, 39(3), 777-789.

Bandura, A. (1986). Social foundations of thought and action: A social cognitive theory. New York, NY: Prentice-Hall.

Bandura, A. (1991). Social cognitive theory of self-regulation. Organizational Behavior and Human Decision Processes, 50(2), 248-287.

Bandura, A., Cioffi, D., Taylor, C. B., \& Brouillard, M. E. (1988). Perceived self-efficacy in coping with cognitive stressors and opioid activation. Journal of Personality and Social Psychology, 55(3), 479-488.

Barnoy, S., Ofra, L., \& Bar-Tal, Y. (2012). What makes patients perceive their health care worker as an epistemic authority? Nursing Inquiry, 19(2), 128-133. 
Bertrand, J., Floyd, R.L., Weber, M.K., O'Connor, M., Riley, E.P., Johnson, K.A., Cohen, D.E., National Task Force on FAS/FAE. (2004). Fetal Alcohol Syndrome: Guidelines for Referral and Diagnosis. Atlanta, GA: Centers for Disease Control and Prevention.

Bishop, F., Lewis, G., Harris, S., McKay, N., Prentice, P., Thiel, H. \& Lewith

G.T. (2010) A within-subjects trial to test the equivalence of online and paper outcome measures: the Roland Morris Disability Questionnaire. BMC Musculoskeletal Disorders 11, 113.

Bertrand, J., Floyd, R.L., Weber, M.K., O'Connor, M., Riley, E.P., Johnson, K.A., Cohen, D.E., National Task Force on FAS/FAE. (2004). Fetal Alcohol Syndrome: Guidelines for Referral and Diagnosis. Atlanta, GA: Centers for Disease Control and Prevention.

Bolman, C., de Vries, H., \& van Breukelen G. (2002). Evaluation of a nurse-managed minimalcontact smoking cessation intervention for cardiac inpatients. Health Education Research, 17(1): 99-116.

Boon, B., Risselada, A., Huiberts, A., Riper, H., \& Smit, F. (2011). Curbing alcohol use in male adults through computer generated personalized advice: randomized controlled trial Journal of Medical Internet Research, 13(2), e43.

Brug, J., Glanz, K., Van Assema, P., Kok, G., \& van Breukelen, G.J.P. (1998). The Impact of Computer-Tailored Feedback and Iterative Feedback on Fat, Fruit, and Vegetable Intake. Health Education \& Behavior, 25(4):517-531.

Butters, L., \& Howie, C. A. (1990). Awareness among pregnant women of the effect on the fetus of commonly used drugs. Midwifery, 6(3), 146-154.

C

Casswell, S. (2004). Alcohol brands in young peoples' everyday lives: New developments in marketing. Alcohol and Alcoholism, 39(6), 471-476.

Centers for Disease Control and Prevention (2002). Alcohol use among women of childbearing age--United States, 1991-1999. Jama, 287(16), 2069-2071.

Centers for Disease Control and Prevention (2012). Alcohol use and binge drinking among women of childbearing age - United States, 2006-2010. Morbidity and Mortality Weekly Report, 61, 534-538.

Chang, G. (2004). Screening and brief intervention in prenatal care settings. Alcohol Research \& Health, 28(2), 80-84.

Chang, G., Goetz, M. A., Wilkins-Haug, L., \& Berman, S. (2000). A brief intervention for prenatal alcohol use: an in-depth look. Journal of Substance Abuse Treatment, 18(4), 365-369.

Chang, G., McNamara, T. K., Orav, E. J., Koby, D., Lavigne, A., Ludman, B., et al. (2005). Brief intervention for prenatal alcohol use: a randomized trial. Obstetrics \& Gynecology, 105(5 Pt 1), 991-998.

Chang, G., McNamara, T. K., Orav, E. J., \& Wilkins-Haug, L. (2006a). Alcohol use by pregnant women: partners, knowledge, and other predictors. Journal of Studies on Alcohol, 67(2), 245-251.

Chang, G., McNamara, T.K., Orav, E.J., \& Wilkins-Haug L. (2006b). Brief intervention for prenatal alcohol use: the role of drinking goal selection. Journal of Substance Abuse Treatment, 31(4): 419-424.

Chang, G., McNamara, T. K., Wilkins-Haug, L., \& Orav, E. J. (2007). Estimates of prenatal abstinence from alcohol: a matter of perspective. Addictive Behaviors, 32(8), 1593-1601.

Chang, G., Orav, E. J., Jones, J. A., Buynitsky, T., Gonzalez, S., \& Wilkins-Haug, L. (2011). Selfreported alcohol and drug use in pregnant young women: a pilot study of associated factors and identification. Journal of Addiction Medicine, 5(3), 221-226.

Chang, G., Wilkins-Haug, L., Berman, S., \& Goetz, M. A. (1999). Brief intervention for alcohol use in pregnancy: a randomized trial. Addiction, 94(10), 1499-1508.

Chernoff, G. F. (1980). The fetal alcohol syndrome in mice: maternal variables. Teratology, 22(1), 71-75.

Chudley, A.E., Conry, J., Cook, J.L., Loock, C., Rosales, R., \& LeBlanc, N. (2005). Fetal alcohol spectrum disorder: Canadian guidelines for diagnosis. Canadian Medical Association Journal, 172(5 suppl), S1-S21. 
Cline, R. J. W., \& Haynes, K. M. (2001). Consumer health information seeking on the Internet: the state of the art. Health Education Research, 16(6), 671-692.

Coleman, T. (1996). Sampling for qualitative research using quantitative methods. 2. Characteristics of GPs who agree to videotaping of consultations. Family Practice, 13(6), 531-535.

Coleman, M. A., Coleman, N. C., \& Murray, J. P. (1990). Mutual support groups to reduce alcohol consumption by pregnant women: marketing implications. Health Marketing Quarterly, 7(3-4), 47-63.

Coles, C. (1994). Critical periods for prenatal alcohol exposure. Alcohol Health \& Research World, 18(1), 22-29.

Collins, L.M., \& Lanza S.T. (2010). Latent Class and Latent Transition Analysis: With Applications in the Social, Behavioral, and Health Sciences. John Wiley \& Sons Inc, Hoboken, NJ.

Comasco, E., Hallberg, G., Helander, A., Oreland, L., \& Sundelin-Wahlsten, V. (2012). Alcohol Consumption Among Pregnant Women in a Swedish Sample and Its Effects on the Newborn Outcomes. Alcoholism: Clinical and Experimental Research, 36(10), 1779-1786.

Cremers, H. P., Mercken, L., Crutzen, R., Willems, P., de Vries, H., \& Oenema, A. (2014). Do email and mobile phone prompts stimulate primary school children to reuse an Internetdelivered smoking prevention intervention? Journal of Medical Internet Research, 16(3), e86.

Cunningham, J. A., Humphreys, K., \& Koski-Jannes, A. (2000). Providing personalized assessment feedback for problem drinking on the Internet: a pilot project. Journal of Studies on Alcohol and Drugs, 61(6), 794-798.

Cutrona, C. E. \& Suhr, J.A. (1992). Controllability of Stressful Events and Satisfaction With Spouse Support Behaviors. Communication Research, 19(2): 154-174.

Cutrona, C. E. \& Suhr, J.A. (1994). Social support communication in the context of marriage: An analysis of couples' supportive interactions. In B. R. Burleson, T. L. Albrecht and I. G. Sarason (Eds.), Communication of social support: Messages, interactions, relationships, and community. (pp. 113-135). Thousand Oaks (Calif): Sage.

D

De Boer, J., \& Zeeman, K. (2008). Prenatale verloskundige begeleiding. Aanbevelingen voor ondersteuning, interactie en voorlichting [Antenatal Support. Recommendations for Support, Interaction and Information]. Utrecht: Royal Organisation of Midwives (in Dutch).

De Jonge, G. A., \& van der Klaauw, M.M. (1986). Onderzoek alcoholgebruik bij zwangere vrouwen [Research alcohol use among pregnant women]. Leiden, the Netherlands: Instituut voor Praeventieve Gezondheidszorg (in Dutch).

De Jonge, A. (2005). KNOV standpunt preconceptiezorg [KNOV standpoint preconception care]. Bilthoven: Royal Dutch Organisation of Midwives (in Dutch).

De Vries, H., Backbier, E., Dijkstra, M., Van Breukelen, G., Parcel, G., \& Kok, G. (1994). A Dutch social influence smoking prevention approach for vocational school students. Health Education Research, 9(3), 365-374.

De Vries, H., Backbier, E., Kok, G., \& Dijkstra, M. (1995). The Impact of Social Influences in the Context of Attitude, Self-Efficacy, Intention, and Previous Behavior as Predictors of Smoking Onset. Journal of Applied Social Psychology, 25(3), 237-257.

De Vries, H., Bakker, M., Mullen, P. D., \& van Breukelen, G. (2006a). The effects of smoking cessation counselling by midwives on Dutch pregnant women and their partners. Patient Education and Counselling, 63(1-2), 177-187.

De Vries, H., \& Brug, J. (1999). Computer-tailored interventions motivating people to adopt health promoting behaviours: introduction to a new approach. Patient Education and Counselling, 36, 99 - 105.

De Vries, H., Dijkstra, M., \& Kuhlman, P. (1988). Self-efficacy: the third factor besides attitude and subjective norm as a predictor of behavioural intentions. Health Education Research, 3(3), 273-282. 
De Vries, H., Kremers, S. P., Smeets, T., Brug, J., \& Eijmael, K. (2008). The effectiveness of tailored feedback and action plans in an intervention addressing multiple health behaviors. American Journal of Health Promotion, 22(6), 417-425.

De Vries, H., Logister, M., Krekels, G., Klaasse, F., Servranckx, V., \& van Osch, L. (2012). Internet based Computer Tailored Feedback on Sunscreen Use. Journal of Medical Internet Research, 14(2), e48.

De Vries, H., Mesters, I., Riet, J. V., Willems, K., \& Reubsaet, A. (2006b). Motives of Belgian adolescents for using sunscreen: the role of action plans. Cancer Epidemiology, Biomarkers and Prevention, 15(7), 1360-1366.

De Vries, H., Mesters, I., van de Steeg, H., \& Honing, C. (2005). The general public's information needs and perceptions regarding hereditary cancer: an application of the Integrated Change Model. Patient Education Counselling, 56, 154-165.

De Vries, H., \& Mudde, A. N. (1998). Predicting stage transitions for smoking cessation applying the attitude-social influence-efficacy model. Psychology \& Health, 13(2), 369 - 385.

De Vries, H., Mudde, A., Dijkstra, A., \& Willemsen, M. (1998). Differential beliefs, perceived social influences and self-efficacy expectations among smokers in various motivational phases. Preventive Medicine, 27, 681 - 689.

De Vries, H., Mudde, A., Leijs, I., Charlton, A., Vartiainen, E., Buijs, G., et al. (2003). The European Smoking Prevention Framework Approach (EFSA): an example of integral prevention. Health Education Research, 18(5), 611-626.

Dehle, C., Larsen, D., \& Landers, J. E. (2001). Social Support in Marriage. American Journal of Family Therapy, 29(4), 307-324.

Del Boca, F.K., Darkes, J. (2003). The validity of self-reports of alcohol consumption: state of the science and challenges for research. Addiction, 98, 1-12.

Den Blijker, J. (2010). Advies over alcohol aan zwangere te vrijblijvend [Alcohol advice for pregnant women too optional]. Trouw, 9 september 2010. http://www.webcitation. org/6cchu5sv2.

DePue, J. D., Goldstein, M. G., Schilling, A., Reiss, P., Papandonatos, G., Sciamanna, C., et al. (2002). Dissemination of the AHCPR clinical practice guideline in community health centres. Tobacco Control, 11(4), 329-335.

Devries, K.M., Child, J.C., Bacchus, L.J., Mak, J., Falder, G., Graham, K., et al. (2014). Intimate partner violence victimization and alcohol consumption in women: a systematic review and meta-analysis. Addiction, 109(3):379-391.

Di Matteo, M. R., Robinson, J. D., Heritage, J., Tabbarah, M., \& Fox, S. A. (2003). Correspondence Among Patients' Self-Reports, Chart Records, and Audio/Videotapes of Medical Visits. Health Communication, 15(4), 393-413.

Dijkstra, A. (2005). Working mechanisms of computer-tailored health education: evidence from smoking cessation. Health Education Research, 20(5), 527-539.

Dijkstra, A., de Vries, H., \& Bakker, M. (1996). Pros and cons of quitting, self-efficacy, and the stages of change in smoking cessation. Journal of Consulting and Clinical Psychology, 64(4):758-763.

Dijkstra, A., De Vries, H., \& Roijackers, J. (1998a). Long-term effectiveness of computer-generated tailored feedback in smoking cessation. Health Education Research, 13(2):207-214.

Dijkstra, A., De Vries, H., Roijackers, J., \& van Breukelen, G. (1998b). Tailoring information to enhance quitting in smokers with low motivation to quit: three basic efficacy questions. Health Psychology, 17(6):513-519.

Donnelly, J. C., Cooley, S. M., Walsh, T. A., Sarkar, R., Durnea, U., \& Geary, M. P. (2008). Illegal drug use, smoking and alcohol consumption in a low-risk Irish primigravid population. Journal of Perinatal Medicine, 36(1), 70-72.

Driscoll, C.D., Streissguth, A.P., \& Riley, E.P. (1990). Prenatal alcohol exposure: Comparability of effects in humans and animal models. Neurotoxicology and Teratology, 12(3), 231-237. 


\section{E}

Elfeddali, I., Bolman, C., Candel, M.J., Wiers, R.W., \& de Vries, H. (2012). Preventing smoking relapse via Web-based computer-tailored feedback: a randomized controlled trial. Journal of Medical Internet Research, 14(4):e109.

Elfeddali, I., Bolman, C., Candel, M.J., Wiers, R.W., \& de Vries, H. (2011). The role of self-efficacy, recovery self-efficacy, and preparatory planning in predicting short-term smoking relapse. British Journal of Health Psychology, 17(1): 185-201.

Enders, C.K. (2010). Applied Missing Data Analysis. New York: Guilford Press.

\section{F}

Field, A. (2005). Discovering statistics using SPSS. London: Sage Publications Ltd.

Floyd, R. L., Decouflé, P., \& Hungerford, D. W. (1999). Alcohol use prior to pregnancy recognition. American Journal of Preventive Medicine, 17(2), 101-107.

Foxcroft, D. (2013). Can Prevention Classification be Improved by Considering the Function of Prevention? Prevention Science, 1-5.

Francis, J. J., Johnston, M., Robertson, C., Glidewell, L., Entwistle, V., Eccles, M. P., et al. (2010). What is an adequate sample size? Operationalising data saturation for theory-based interview studies. Psychology \& Health, 25(10), 1229-1245.

\section{G}

Gebara, C.F., Bhona, F.M., Ronzani, T., Lourenco, L., \& Noto, A. (2013). Brief intervention and decrease of alcohol consumption among women: a systematic review. Substance Abuse Treatment, Prevention and Policy, 8:31.

Ginsberg, D., Hall, S. M., \& Rosinski, M. (1991). Partner interaction and smoking cessation: A pilot study. Addictive Behaviors, 16(5), 195-202.

Gollwitzer, P. M. (1999). Implementation intentions: Strong effects of simple plans. American Psychologist, 54(7), 493-503.

Goodlett, C. R., \& Horn, K. H. (2001). Mechanisms of alcohol-induced damage to the developing nervous system. Alcohol Research \& Health, 25(3), 175-184.

Goransson, M., Magnusson, A., Bergman, H., Rydberg, U., \& Heilig, M. (2003). Fetus at risk: prevalence of alcohol consumption during pregnancy estimated with a simple screening method in Swedish antenatal clinics. Addiction, 98(11), 1513-1520.

Gosling, S.D., Vazire, S., Srivastava, S., \& John, O.P. (2004) Should we trust web-based studies? A comparative analysis of six preconceptions about internet questionnaires. American Psychologist, 59, 93-104.

Gottlieb, B. H. \& Bergen, A.E. (2010). Social support concepts and measures. Journal of Psychosomatic Research, 69(5), 511-520.

Gruenewald, P. J. (2007). The spatial ecology of alcohol problems: niche theory and assortative drinking. Addiction, 102(6), 870-878.

\section{H}

Hailes, J. F., \& Wellard, S. J. (2000). Support for breastfeeding in the first postpartum month: perceptions of breastfeeding women. Breastfeeding Review, 8(3), 5-9.

Hajek, P., West, R., Lee, A., Foulds, J., Owen, L., Eiser, J. R., et al. (2001). Randomized controlled trial of a midwife-delivered brief smoking cessation intervention in pregnancy. Addiction, 96(3), 485-494.

Hales, S.D. (1996). Abortion and fathers' rights. In: J.M. Humber \& R.F. Almeder (Eds) Biomedical Ethics Reviews: Reproduction, Technology, and Rights, pp. 5-26. Humana Press, Totowa, NJ.

Hammond, D., Fong, G. T., McDonald, P. W., Brown, K. S., Cameron, R. (2006). Showing leads to doing: graphic cigarette warning labels are an effective public health policy. European Journal of Public Health, 16(2), 223-224; author reply 225. 
Handmaker, N. S., Miller, W. R., \& Manicke, M. (1999). Findings of a pilot study of motivational interviewing with pregnant drinkers. Journal of Studies on Alcohol and Drugs, 60(2), 285287.

Hauck, Y. L., Graham-Smith, C., McInerney, J., \& Kay, S. (2011). Western Australian women's perceptions of conflicting advice around breast feeding. Midwifery, 27(5), e156-162.

Hawkins, R. P., Kreuter, M., Resnicow, K., Fishbein, M., \& Dijkstra, A. (2008). Understanding tailoring in communicating about health. Health Education Research, 23(3), 454-466.

Hayes, A., \& Matthes, J. (2009). Computational procedures for probing interactions in OLS and logistic regression: SPSS and SAS implementations. Behavior Research Methods, 41(3), 924936.

Haynes, G., Dunnagan, T., \& Christopher, S. (2003). Determinants of alcohol use in pregnant women at risk for alcohol consumption. Neurotoxicology and Teratology, 25(6), 659-666.

Health Council of the Netherlands (2005). Risks of alcohol consumption related to conception, pregnancy and breastfeeding (No. publication no. 2004/22). The Hague: Health Council of the Netherlands.

Holmqvist, M., \& Nilsen, P. (2010). Approaches to assessment of alcohol intake during pregnancy in Swedish maternity care--a national-based investigation into midwives' alcohol-related education, knowledge and practice. Midwifery, 26(4), 430-434.

Hoving, C., Mudde, A.N., \& de Vries, H. (2007a). Effect of recruitment method and setting on the composition of samples consisting of adult smokers. Patient Education and Counselling, 65(1):79-86

Hoving, E.F., Mudde, A.N., \& de Vries H. (2006a). Predictors of smoking relapse in a sample of Dutch adult smokers; the roles of gender and action plans. Addictive Behaviors, 31, 11771189.

Hoving, E. F., Mudde, A. N., \& de Vries, H. (2006b). Smoking and the O pattern; predictors of transitions through the stages of change. Health Education Research, 21(3), 305-314.

Hoving, C., Mudde, A.N., Dijk, F., \& de Vries, H. (2010). Effectiveness of a smoking cessation intervention in Dutch pharmacies and general practices. Health Education, 110(1):17-29.

Hoving, C., Reubsaet, A., \& de Vries, H. (2007b). Predictors of smoking stage transitions for adolescent boys and girls. Preventive Medicine, 44(6), 485-489.

ICAP. (2012). Health warning labels. http://www.icap.org/table/HealthWarningLabels.

Idanpaan-Heikkila, J., Jouppila, P., Akerblom, H. K., Isoaho, R., Kauppila, E., \& Koivisto, M. (1972). Elimination and metabolic effects of ethanol in mother, fetus, and newborn infant. American Journal of Obstetrics \& Gynecology, 112(3), 387-393.

\section{J}

Jacobson, S. W., Chiodo, L. M., Sokol, R. J., \& Jacobson, J. L. (2002). Validity of maternal report of prenatal alcohol, cocaine, and smoking in relation to neurobehavioral outcome. Pediatrics, 109(5), 815-825.

Jago, R., \& Sebire, S. J. (2012). Publishing pilot and feasibility evaluations of behavioural interventions: Implications for Preventive Medicine. Preventive Medicine, 55(6), 548-549.

Janz, N. K., \& Becker, M. H. (1984). The Health Belief Model: a decade later. Health Education Quarterly, 11(1), 1-47.

Jentink, J., Zetstra-van der Woude, A. P., Bos, J., \& de Jong-van den Berg, L. T. W. (2011). Evaluation of the representativeness of a Dutch non-malformed control group for the general pregnant population: are these controls useful for EUROCAT? Pharmacoepidemiology and Drug Safety, 20(11), 1217-1223.

Joffe, A., \& Radius, S. M. (1991). Health Counselling of Adolescents. Pediatrics in Review, 12(11), 344-351.

Johnson, M., Jackson, R., Guillaume, L., Meier, P., \& Goyder, E. (2011). Barriers and facilitators to implementing screening and brief intervention for alcohol misuse: a systematic review of qualitative evidence. Journal of Public Health, 33(3):412-421. 
Johnson, M., Robinson, R., Corey, S., Dewane, S., Brems, C., \& Diane Casto, L. (2010). Knowledge, attitudes, and behaviors of health, education, and service professionals as related to fetal alcohol spectrum disorders. International Journal of Public Health, 55(6), 627-635.

Jones, T.B., Bailey, B.A., \& Sokol, R.J. (2013). Alcohol use in pregnancy: insights in screening and intervention for the clinician. Clinical Obstetrics and Gynecology, 56(1), 114-123.

Jones, S. C., Eval, M., Telenta, J., Cert, G., Shorten, A., \& Johnson, K. (2011). Midwives and pregnant women talk about alcohol: what advice do we give and what do they receive? Midwifery, 27(4), 489-496.

\section{K}

Kateman, H., \& Herschderfer, K. (2005). Multidisciplinary collaborative primary maternity care project. Current Practice in Europe and Australia. Den Haag: International Confederation of Midiwives.

Kerssemakers, R., van Meerten, R., Noorlander, E., \& Vervaeke, H. (Red.) (2008). Drugs en alcohol. Gebruik, misbruik en verslaving [Drugs and alcohol. Use, misuse and addiction]. Houten: Bohn Stafleu van Loghum (in Dutch).

Kesmodel, U., Kesmodel, P. S., Larsen, A., \& Secher, N. J. (2003). Use of alcohol and illicit drugs among pregnant Danish women, 1998. Scandinavian Journal of Public Health, 31(1), 5-11.

Kesmodel, U., \& Schioler Kesmodel, P. (2002). Drinking during pregnancy: attitudes and knowledge among pregnant Danish women, 1998. Alcoholism: Clinical and Experimental Research, 26(10), 1553-1560.

Kesmodel, U. S., \& Kesmodel, P. S. (2011). Alcohol in pregnancy: attitudes, knowledge, and information practice among midwives in Denmark 2000 to 2009. Alcoholism: Clinical and Experimental Research, 35(12), 2226-2230.

Kissinger, P., Rice, J., Farley, T., Trim, S., Jewitt, K., Margavio, V., et al. (1999). Application of Computer-assisted Interviews to Sexual Behavior Research. American Journal of Epidemiology, 149(10), 950-954.

Knops-Dullens, T., de Vries, N., \& de Vries, H. (2007). Reasons for non-attendance in cervical cancer screening programmes: an application of the Integrated Model for Behavioural Change. European Journal of Cancer Prevention, 16(5), 436-445.

Kools, E. J., Thijs, C., \& de Vries, H. (2005a). The behavioral determinants of breast-feeding in The Netherlands: predictors for the initiation of breast-feeding. Health Education \& Behavior, 32(6), 809-824.

Kools, E. J., Thijs, C., Kester, A. D. M., van den Brandt, P. A., \& de Vries, H. (2005b). A breastfeeding promotion and support program a randomized trial in the Netherlands. Preventive Medicine, 40(1), 60-70.

Koordeman, R., Anschutz, D. J., \& Engels, R. C. M. E. (2012). The Effect of Alcohol Advertising on Immediate Alcohol Consumption in College Students: An Experimental Study. Alcoholism: Clinical and Experimental Research, 36(5), 874-880.

Koshy, P., Mackenzie, M., Tappin, D., \& Bauld, L. (2010). Smoking cessation during pregnancy: the influence of partners, family and friends on quitters and non-quitters. Health and Social Care in the Community, 18(5), 500-510.

Koski-Jannes, A., Cunningham, J. A., Tolonen, K., \& Bothas, H. (2007). Internet-based selfassessment of drinking -- 3-month follow-up data. Addictive Behaviors, 32(3), 533-542.

Krebs, P., Prochaska, J. O., \& Rossi, J. S. (2010). A meta-analysis of computer-tailored interventions for health behavior change. Preventive Medicine, 51(3-4), 214-221.

Kreuter, M., Farrell, D., Olevitch, L., Brennan, L. (1999). Tailoring Health Messages: Customizing Communication with Computer Technology. Mahwah, NJ: Lawrence Erlbaum Associates.

Krippendorff, K. (1980). Content Analysis: An Introduction to Its Methodology. Newbury Park, CA: Sage.

\section{$\mathrm{L}$}

Lange, S., Shield, K., Koren, G., Rehm, J., \& Popova, S. (2014). A comparison of the prevalence of prenatal alcohol exposure obtained via maternal self-reports versus meconium testing: a systematic literature review and meta-analysis. BMC Pregnancy Childbirth, 14(1), 127. 
Langeland, W., Draijer, N., \& van den Brink, W. (2003). Assessment of lifetime physical and sexual abuse in treated alcoholics: Validity of the Addiction Severity Index. Addictive Behaviors, 28(5), 871-881.

Lanting, C. I., Buitendijk, S. E., Crone, M. R., Segaar, D., Bennebroek Gravenhorst, J., \& van Wouwe, J. P. (2009). Clustering of Socioeconomic, Behavioural, and Neonatal Risk Factors for Infant Health in Pregnant Smokers. PLoS One, 4(12), e8363.

Lechner, L., Bolman, C., \& Van Dijke, M. (2006). Factors related to misperception of physical activity in The Netherlands and implications for health promotion programmes. Health Promotion International, 21(2), 104-112.

Lechner, L., \& de Vries, H. (2002). Sunbed use at home: risk behaviour and psychosocial determinants. European Journal of Cancer Prevention, 11(4), 333-341.

Lemmens, P., Tan, E. S., \& Knibbe, R. A. (1992). Measuring quantity and frequency of drinking in a general population survey: a comparison of five indices. Journal of Studies on Alcohol and Drugs, 53(5), 476-486.

Livingston, M., Chikritzhs, T., \& Room, R. (2007). Changing the density of alcohol outlets to reduce alcohol-related problems. Drug and Alcohol Review, 26(5), 557-566.

Locke, E. A., \& Latham, G. P. (2002). Building a practically useful theory of goal setting and task motivation. A 35-year odyssey. American Psychologist, 57(9), 705-717.

Lumley, J., Chamberlain, C., Dowswell, T., Oliver, S., Oakley, L., \& Watson, L. (2009). Interventions for promoting smoking cessation during pregnancy. Cochrane Database of Systematic Reviews(3), CD001055.

\section{M}

Marlatt, G. A., Baer, J.S., \& Quigley, L.A. (1995). Self-efficacy and addictive behavior. In A. Bandura (Ed.), Self-efficacy in changing societies (pp. 289 - 315). New York: Cambridge University Press.

Marshall, M.N. (1996). Sampling for qualitative research. Family Practice, 13(6), 522-526.

Martin, L., McNamara, M., Milot, A., Halle, T., \& Hair, E. (2007). The Effects of Father Involvement during Pregnancy on Receipt of Prenatal Care and Maternal Smoking. Maternal and Child Health Journal, 11(6), 595-602.

Mavroforou, A., Koumantakis, E., \& Michalodimitrakis E. (2010) Do men have rights in abortion? The Greek view. Medicine and Law 29, 77-85.

May, P. A., Daniela, F., Gossage, J. P., Wendy, O. K., Hoyme, H. E., Luther, K. R., et al. (2006). Epidemiology of FASD in a Province in Italy: Prevalence and Characteristics of Children in a Random Sample of Schools. Alcoholism: Clinical and Experimental Research, 30(9), 1562-1575.

May, P. A., Fiorentino, D., Coriale, G., Kalberg, W. O., Hoyme, H. E., Aragon, A. S., et al. (2011). Prevalence of children with severe fetal alcohol spectrum disorders in communities near rome, Italy: new estimated rates are higher than previous estimates. International Journal of Environmental Research and Public Health, 8(6), 2331-2351.

May, P. A., Gossage, J. P., Marais, A. S., Hendricks, L. S., Snell, C. L., Tabachnick, B. G., et al. (2008) Maternal Risk Factors for Fetal Alcohol Syndrome and Partial Fetal Alcohol Syndrome in South Africa: A Third Study. Alcoholism: Clinical and Experimental Research, 32(5), 738-753.

McBride, C. M., Baucom, D. H., Peterson, B. L., Pollak, K. I., Palmer, C., Westman, E., et al. (2004). Prenatal and Postpartum Smoking Abstinence: A Partner-Assisted Approach. American Journal of Preventive Medicine, 27(3), 232-238.

McLellan, A., Lewis, D. C., O’Brien, C. P., Kleber H. D. (2000). Drug dependence, a chronic medical illness: Implications for treatment, insurance, and outcomes evaluation. JAMA: The Journal of the American Medical Association, 284(13), 1689-1695.

McKay, S., 2004. Models of midwifery care: Denmark, Sweden, and the

Netherlands. In: Van Teijlingen, G.W.L.E.R., McCaffery, P., Porter, M. (Eds.), Midwifery and the Medicalization of Childbirth: Comparative Perspectives. Nova Science Publishers, Inc. New York, pp. 155-162.

McNamara, T. K., Orav, E. J., Wilkins-Haug, L., \& Chang, G. (2006). Social support and prenatal alcohol use. Journal of Women's Health (Larchmt), 15(1), 70-76. 
Miller, W.R., \& Rollnick, S. (1991). Motivational interviewing: Preparing people to change addictive behavior. New York, NY: The Guilford Press.

Monarrez-Espino, J., Liu, B., Greiner, F., Bremberg, S., Galanti, R. (2014). Systematic review of the effect of pictorial warnings on cigarette packages in smoking behavior. American Journal of Public Health, 104(10), e11-30.

Moore, G., Audrey, S., Barker, M., Bond, L., Bonell, C., Cooper, C., et al. (2014). Process evaluation in complex public health intervention studies: the need for guidance. Journal of Epidemiology \& Community Health, 68(2), 101-102.

Morrow-Tlucak, M., Ernhart, C. B., Sokol, R. J., Martier, S., \& Ager, J. (1989). Underreporting of alcohol use in pregnancy: relationship to alcohol problem history. Alcoholism: Clinical and Experimental Research, 13(3), 399-401.

Murray, E. (2007). Using the internet for research: results at a keystroke. British Journal of General Practice, 57, 939-940.

\section{$\mathrm{N}$}

Nagin, D.S. (2005). Group-based Modelling of Development over the Life Course. Harvard University Press, Cambridge, MA.

Nathanson, V., Jayesinghe, N., \& Roycroft, G. (2007). Is it all right for women to drink small amounts of alcohol in pregnancy? No. Bmj, 335(7625), 856-857.

National Health and Medical Research Council (2009). Australian Guidelines to Reduce Health Risks from Drinking Alcohol. Commonwealth of Australia.

Niccols, A. (2007). Fetal alcohol syndrome and the developing socio-emotional brain. Brain and Cognition, 65(1), 135-142.

Nilsen, P. (2009). Brief alcohol intervention to prevent drinking during pregnancy: an overview of research findings. Current Opinion in Obstetrics and Gynecology, 21(6):496-500.

Nilsen, P., Holmqvist, M., Hultgren, E., Bendtsen, P., \& Cedergren, M. (2008). Alcohol use before and during pregnancy and factors influencing change among Swedish women. Acta Obstetricia et Gynecologica Scandinavica, 87(7), 768-774.

Norman, G. (2010) Likert scales, levels of measurement and the 'laws' of statistics. Advances in Health Science Education, 15, 625-632.

\section{0}

O'Connor, M. J., \& Whaley, S. E. (2007). Brief intervention for alcohol use by pregnant women. American Journal of Public Health, 97(2), 252-258.

Ockene, J.K., Ma, Y., Zapka, J.G., Pbert, L.A., Goins, K.V., \& Stoddard, A.M. (2002). Spontaneous cessation of smoking and alcohol use among low-income pregnant women. American journal of preventive medicine, 23(3), 150-159.

Oenema, A., \& Brug, J. (2003). Feedback strategies to raise awareness of personal dietary intake: results of a randomized controlled trial. Preventive Medicine, 36(4), 429-439.

Oenema, A., Tan, F., \& Brug, J. (2005). Short-term efficacy of a web-based computer-tailored nutrition intervention: main effects and mediators. Annals of Behavioral Medicine, 29(1), 54-63.

O'Leary, C.M., Heuzenroeder, L., Elliott, E.J., \& Bower, C. (2007). A review of policies on alcohol use during pregnancy in Australia and other English-speaking countries, 2006. Medical Journal of Australia, 186(9), 466-471.

O'Leary, C.M. (2004). Fetal alcohol syndrome: diagnosis, epidemiology, and developmental outcomes. Journal of Paediatrics and Child Health, 40(1-2), 2-7.

Olsen, J. (1993). Predictors of smoking cessation in pregnancy. Scandinavian Journal of Social Medicine, 21(3), 197-202. 


\section{$\mathbf{P}$}

Park, E. W., Tudiver, F. G., \& Campbell, T. (2012). Enhancing partner support to improve smoking cessation. Cochrane Database of Systematic Reviews, 7, CD002928.

Patton, M. Q. (1990). Qualitative evaluation and research methods (2nd ed.). Newbury Park, CA: Sage.

Patra, J., Bakker, R., Irving, H., Jaddoe, V.W., Malini, S., \& Rehm, J. (2011).

Dose-response relationship between alcohol consumption before and during pregnancy and the risks of low birthweight, preterm birth and small for gestational age (SGA)-a systematic review and meta-analyses. Bjog, 118(12), 1411-1421.

Peadon, E., Payne, J., Henley, N., D’Antoine, H., Bartu, A., O’Leary, C., et al. (2011). Attitudes and behaviour predict women's intention to drink alcohol during pregnancy: the challenge for health professionals. BMC Public Health, 11(1), 584.

Perham-Hester, K. A., \& Gessner, B. D. (1997). Correlates of drinking during the third trimester of pregnancy in Alaska. Maternal and Child Health Journal, 1(3), 165-172.

Peristat II. (2008). EURO-PERISTAT project in collaboration with SCPE,

EUROCAT and EURONEONET. European perinatal health report. Better statistics for better health for pregnant women and their babies in 2004. Available: www.europeristat.com

Petkovic, G., \& Barisic, I. (2010). FAS prevalence in a sample of urban schoolchildren in Croatia. Reproductive Toxicology, 29(2), 237-241.

Petkovic, G., \& Barisic, I. (2013). Prevalence of Fetal Alcohol Syndrome and Maternal Characteristics in a Sample of Schoolchildren from a Rural Province of Croatia. International Journal of Environmental Research and Public Health, 10(4), 1547-1561.

Pfinder, M., Kunst, A., Feldmann, R., van Eijsden, M., \& Vrijkotte, T. G. M. (2013). Preterm birth and small for gestational age in relation to alcohol consumption during pregnancy: stronger associations among vulnerable women? results from two large Western-European studies. BMC Pregnancy and Childbirth, 13(1), 49.

Phares, T. M., Morrow, B., Lansky, A., Barfield, W.D., Prince, C.B., Marchi, K.S., Braveman, P.A., Williams, L.M., \& Kinniburgh, B. (2004). Surveillance for disparities in maternal healthrelated behaviors--selected states, Pregnancy Risk Assessment Monitoring System (PRAMS), 2000-2001. Morbidity and Mortality Weekly Report Surveillance Summary, 53(4), 1-13.

Pieterse, M.E., Seydel, E.R., de Vries, H., Mudde, A.N., \& Kok, G.J. (2001). Effectiveness of a Minimal Contact Smoking Cessation Program for Dutch General Practitioners: A Randomized Controlled Trial. Preventive Medicine, 32(2): 182-190.

Popova, S., Lange, S., Burd, L., \& Rehm, J. (2013). Health Care Burden and Cost Associated with Fetal Alcohol Syndrome: Based on Official Canadian Data. PLoS One, 7(8), e43024.

Prochaska, J.O., \& DiClemente, C.C. (1983). Stages and processes of self-change of smoking: toward an integrative model of change. Journal of Consulting and Clinical Psychology, 51, 390-395.

Prochaska, J. O., \& Velicer, W. F. (1997). The transtheoretical model of health behavior change. American Journal of Health Promotion, 12(1), 38-48.

\section{$\mathbf{R}$}

Raymond, N., Beer, C., Glazebrook, C., \& Sayal, K. (2009). Pregnant women's attitudes towards alcohol consumption. BMC Public Health, 9, 175.

Reynolds, K. D., Coombs, D. W., Lowe, J. B., Peterson, P. L., \& Gayoso, E. (1995). Evaluation of a Self-Help Program to Reduce Alcohol Consumption among Pregnant Women. International Journal of Mental Health and Addiction, 30(4), 427 - 443.

Riper, H., Kramer, J., Smit, F., Conijn, B., Schippers, G., \& Cuijpers, P. (2008). Web-based self-help for problem drinkers: a pragmatic randomized trial. Addiction, 103(2), 218-227.

Riper, H., Kramer, J., Conijn, B., Smit, F., Schippers, G., \& Cuijpers, P. (2009). Translating Effective Web-Based Self-Help for Problem Drinking Into the Real World. Alcoholism: Clinical and Experimental Research, 33(8), 1401-1408. 
Room, R., Jernigan, D., Carlini-Marlatt, B., Gureje, O., Makela, K., Marshall, M., et al. (2002). Alcohol in developing societies: A public health approach. Helsinki: Finnish foundation for Alcohol Studies/World Health Organization.

Ruiter, R. A. C., \& Kok, G. (2005). Saying is not (always) doing: cigarette warning labels are useless. European Journal of Public Health, 15(3), 329.

\section{$\mathrm{S}$}

Sayal, K., Heron, J., Golding, J., \& Emond, A. (2007). Prenatal alcohol exposure and gender differences in childhood mental health problems: a longitudinal population-based study. Pediatrics, 119, e426-434.

Schnoll, R. A., Martinez, E., Tatum, K. L., Glass, M., Bernath, A., Ferris, D., et al. (2011). Increased self-efficacy to quit and perceived control over withdrawal symptoms predict smoking cessation following nicotine dependence treatment. Addictive Behaviors, 36(1-2), 144-147.

Schulz, D. N., Candel, M. J., Kremers, S. P., Reinwand, D. A., Jander, A., \& de Vries, H. (2013). Effects of a web-based tailored intervention to reduce alcohol consumption in adults: randomized controlled trial. Journal of Medical Internet Research, 15(9), e206.

Schulz, D. N., Smit, E. S., Stanczyk, N. E., Kremers, S. P., de Vries, H., \& Evers, S. M. (2014). Economic evaluation of a web-based tailored lifestyle intervention for adults: findings regarding cost-effectiveness and cost-utility from a randomized controlled trial. Journal of Medical Internet Research, 16(3), e91.

Schwarzer, R., Lippke, S., \& Luszczynska, A. (2011). Mechanisms of health behavior change in persons with chronic illness or disability: the Health Action Process Approach (HAPA). Rehabilitation Psychology, 56(3), 161-170.

Schwarzer, R., \& Renner, B. (2000). Social-cognitive predictors of health behavior: Action selfefficacy and coping self-efficacy. Health Psychology, 19(5), 487-495.

Scott, D. M., \& Taylor, R. E. (2007). Health-related effects of genetic variations of alcoholmetabolizing enzymes in African Americans. Alcohol Research \& Health, 30(1), 18-21.

Segaar, D., Bolman, C., Willemsen, M., \& De Vries, H. (2007). Identifying determinants of protocol adoption by midwives: a comprehensive approach. Health Education Research, 22(1), 1426.

Singal, A. G., Higgins, P. D. R., \& Waljee, A. K. (2014). A Primer on Effectiveness and Efficacy Trials. Clinical and Translational Gastroenterology, 5, e45.

Skagerstrom, J., Chang, G., \& Nilsen, P. (2011). Predictors of drinking during pregnancy: a systematic review. Journal of Women's Health (Larchmt), 20(6), 901-913.

Skagerström, J., Johansson, A. L., Holmqvist, M., Envall, E. K., \& Nilsen, P. (2012). Towards improved alcohol prevention in Swedish antenatal care? Midwifery, 28(3), 314-320.

Smit, E. S., de Vries, H., \& Hoving, C. (2012). Effectiveness of a web-based multiple tailored smoking cessation program: a randomized controlled trial among dutch adult smokers. Journal of Medical Internet Research, 14(3), e82.

Smit, E.S., De Vries, H., \& Hoving C. (2013a). Determinants of practice nurses' intention to implement a new smoking cessation intervention: the importance of attitude and innovation characteristics. Journal of Advanced Nursing, 69, 2665-2674

Smit, E. S., Evers, S. M., de Vries, H., \& Hoving, C. (2013b). Cost-effectiveness and cost-utility of Internet-based computer tailoring for smoking cessation. Journal of Medical Internet Research, 15(3), e57.

Smit, F., Lokkerbol, J., Riper, H., Majo, M. C., Boon, B., \& Blankers, M. (2011). Modeling the costeffectiveness of health care systems for alcohol use disorders: how implementation of eHealth interventions improves cost-effectiveness. Journal of Medical Internet Research, 13(3), e56.

Snyder, L. B., Milici, F., Slater, M., Sun, H., \& Strizhakova, Y. (2006). Effects of alcohol advertising exposure on drinking among youth. Archives of Pediatrics and Adolescent Medicine Journal, 160(1), 18-24.

Sobell, L.C., \& Sobell, M.B. (1992). Timeline followback: A technique for assessing self-reported ethanol consumption. In: Allen J, Litten RZ, editors. Measuring alcohol consumption: Psychosocial and biological methods. Totowa, NJ: Humana Press. p. 41-72. 
Sohanpal, R., Hooper, R., Hames, R., Priebe, S., \& Taylor, S. (2012). Reporting participation rates in studies of non-pharmacological interventions for patients with chronic obstructive pulmonary disease: a systematic review. Systematic Reviews, 1(1), 66.

Sokol, R.J., Martier, S.S., \& Ager, J.W. (1989). The T-ACE questions: practical prenatal detection of risk-drinking. American Journal of Obstetrics \& Gynecology, 160(4): 863-868; discussion 868-870.

Stade, B. C., Bailey, C., Dzendoletas, D., Sgro, M., Dowswell, T., \& Bennett, D. (2009). Psychological and/or educational interventions for reducing alcohol consumption in pregnant women and women planning pregnancy. Cochrane Database of Systematic Reviews(2), CD004228.

STAP. (2012). www.alcoholenzwangerschap.nl

State Secretary for Health Welfare and Sport M.J. van Rijn (2013). Answer to Parliamentary Questions from MPs Bruins Slot, Dik-Faber and Van der Staaij. (Vol. 2013Z12948). The Hague.

Statistics Netherlands (2013). www.statline.cbs.nl

Statistics Netherlands (2014). http://www.webcitation.org/6S3vohBp5

Steckler, A., McLeroy, K. R., Goodman, R. M., Bird, S. T., \& McCormick, L. (1992). Toward Integrating Qualitative and Quantitative Methods: An Introduction. Health Education \& Behavior, 19(1), 1-8.

Strand, M., Phelan, K. J., \& Donovan, E. F. (2003). Promoting the uptake and use of evidence: an overview of the problem. Clinics in Perinatology, 30(2), 389-402.

Strecher, V.J., Shiffman, S., \& West, R. (2006). Moderators and mediators of a web-based computer-tailored smoking cessation program among nicotine patch users. Nicotine \& Tobacco Research, 8 Suppl 1, S95-101.

Streissguth, A. P., \& O'Malley, K. (2000). Neuropsychiatric implications and long-term consequences of fetal alcohol spectrum disorders. Seminars in Clinical Neuropsychiatry, 5(3), 177-190.

Stuurgroep Zwangerschap en Geboorte (2009). Een goed begin: Veilige zorg rond zwangerschap en geboorte [A good start: Safe care during pregnancy and birth] . Utrecht: Stuurgroep zwangerschap en geboorte.

Sulik, K. K., Cook, C. S., \& Webster, W. S. (1988). Teratogens and craniofacial malformations: relationships to cell death. Development, 103 Suppl, 213-231.

\section{T}

Tarrant, R. C., Younger, K. M., Sheridan-Pereira, M., White, M. J., \& Kearney, J. M. (2010). The prevalence and determinants of breast-feeding initiation and duration in a sample of women in Ireland. Public Health Nutrition, 13(6), 760-770.

Te Poel, F., Bolman, C., Reubsaet, A., de Vries, H. (2009). Efficacy of a single computer-tailored e-mail for smoking cessation: results after 6 months. Health Education Research, 24(6), 930-940.

Testa, M., \& Reifman, A. (1996). Individual differences in perceived riskiness of drinking in pregnancy: antecedents and consequences. Journal of Studies on Alcohol and Drugs, 57(4), 360-367.

Tholen, A. M. J. (1987). Alcohol en zwangerschap [Alcohol and pregnancy]. Assen: GVO Drenthe.

Tideman, R. L., Chen, M. Y., Pitts, M. K., Ginige, S., Slaney, M., \& Fairley, C. K. (2007). A randomised controlled trial comparing computer-assisted with face-to-face sexual history taking in a clinical setting. Sexually Transmitted Infections, 83(1), 52-56.

Torvik, F.A., Røysamb, E., Gustavson, K., Idstad, M., \& Tambs, K. (2013). Discordant and Concordant Alcohol Use in Spouses as Predictors of Marital Dissolution in the General Population: Results from the Hunt Study. Alcoholism: Clinical and Experimental Research, 37(5), 877-84.

Toutain, S. (2010). What women in France say about alcohol abstinence during pregnancy. Drug and Alcohol Review, 29(2), 184-188.

Tweede Kamer. (2010). Parliamentary questions and answers 2010D38996 Aanhangsel Tweede Kamer 2010-2011, nr. 178.

Tweede Kamer. (2012). Parliamentary questions 2012Z21274. 
Tweede Kamer. (2013). Answers to Parliamentary questions Aanhangsel Tweede Kamer 20122013, nr. 1053.

Tweede Kamer. (2015a). Parliamentary questions $2015 Z 14801$.

Tweede Kamer. (2015b). Answers to Parliamentary questions Aanhangsel Tweede Kamer 20152016, nr. 130.

Tzilos, G. K., Sokol, R. J., \& Ondersma, S. J. (2011). A randomized phase I trial of a brief computerdelivered intervention for alcohol use during pregnancy. Journal of Women's Health (Larchmt), 20(10), 1517-1524.

\section{V}

van der Wulp, N.Y., Hoving, C., \& De Vries, H. (2013). A qualitative investigation of alcohol use advice during pregnancy: Experiences of Dutch midwives, pregnant women and their partners. Midwifery, 29(11), e89-e98.

van der Wulp, N.Y., Hoving, C., \& De Vries, H. (2014a). Dutch midwives' experiences with implementing health counselling to prevent prenatal alcohol use. Journal of Clinical Nursing, 23(21-22), 3286-3289.

van der Wulp, N., Hoving, C., \& de Vries, H. (2014b). Partner's influences and other correlates of prenatal alcohol use. Maternal and Child Health Journal 19(4), 908-916.

van Faassen, E., \& Niemela, O. (2011). Biochemistry of prenatal alcohol exposure. Nova Science Publishers.

van Osch, L., Lechner, L., Reubsaet, A., Wigger, S., \& de Vries, H. (2008). Relapse prevention in a national smoking cessation contest: effects of coping planning. British Journal of Health Psychology, 13(Pt 3): 525-535.

van Osch, L., Reubsaet, A., Lechner, L., Beenackers, M., Candel, M., \& de Vries, H. (2009). Planning health behaviour change: Comparing the behavioural influence of two types of self-regulatory planning. British Journal of Health Psychology, 15(Pt 1), 133-149.

van Stralen, M.M., de Vries, H., Mudde, A.N., Bolman, C., \& Lechner, L. (2011). The long-term efficacy of two computer-tailored physical activity interventions for older adults: Main effects and mediators. Health Psychology, 30(4): 442-52.

van 't Riet, J., Ruiter, R. A. C., Werrij, M. Q., \& de Vries, H. (2010). Investigating message-framing effects in the context of a tailored intervention promoting physical activity. Health Education Research, 25(2), 343-354.

van Wieringen, H., Rodrigues Pereira, R., Cobben, J.M., \& Lindhout, D. (2009). Signaleringsonderzoek foetaal alcohol syndroom, Jaarverslag Nederlands SignaleringsCentrum Kindergeneeskunde 2008 [Signalling research foetal alcohol syndrome, Annual report Dutch signalling center pediatrics 2008]. TNO-rapport 2009.068. Leiden: TNO (in Dutch).

Verkerk, P. H., van Noord-Zaadstra, B. M., Florey, C. D., de Jonge, G. A., \& Verloove-Vanhorick, S. P. (1993). The effect of moderate maternal alcohol consumption on birth weight and gestational age in a low risk population. Early Human Development, 32(2-3), 121-129.

\section{W}

Wagenaar, A. C., Salois, M. J., \& Komro, K. A. (2009). Effects of beverage alcohol price and tax levels on drinking: a meta-analysis of 1003 estimates from 112 studies. Addiction, 104(2), 179-190.

Waterson, E. J., Evans, C., \& Murray-Lyon, I. M. (1990). Is pregnancy a time of changing drinking and smoking patterns for fathers as well as mothers? An initial investigation. British Journal of Addiction, 85(3), 389-396.

Whitehead, N., \& Lipscomb, L. (2003). Patterns of alcohol use before and during pregnancy and the risk of small-for-gestational-age birth. American Journal of Epidemiology, 158(7), 654-662.

Wiegers, T. A. (2007). Workload of primary-care midwives. Midwifery, 23(4), 425-432. 
Wiegers, T.A., Van der Velden, L.F.J., \& Hingstman L. (2005). Behoefteraming voor verloskundigen 2004-2015 [Need for Midwives 2004-2015]. Nivel, Utrecht (in Dutch).

Wilkinson, C., \& Room, R. (2009). Warnings on alcohol containers and advertisements: International experience and evidence on effects. Drug and Alcohol Review, 28(4), 426-435.

Wilson, G., McGovern, R., Antony, G., Cassidy, P., Deverill, M., Graybill, E., et al. (2012). Brief intervention to reduce risky drinking in pregnancy: study protocol for a randomized controlled trial. Trials, 13(1), 174.

Wolfberg, A. J., Michels, K. B., Shields, W., O'Campo, P., Bronner, Y., \& Bienstock, J. (2004). Dads as breastfeeding advocates: results from a randomized controlled trial of an educational intervention. American Journal of Obstetrics \& Gynecology, 191(3), 708-712.

World Health Organization (WHO). (2007). WHO Expert Committee on Problems Related to Alcohol Consumption (second report). Geneva: WHO.

\section{Z}

Zammit, S. L., Skouteris, H., Wertheim, E. H., Paxton, S. J., \& Milgrom, J. (2008). Pregnant women's alcohol consumption: the predictive utility of intention to drink and prepregnancy drinking behavior. Journal of Women's Health (Larchmt), 17(9), 1513-1522. 
Summary 
There is accumulating scientific evidence that even low levels of prenatal alcohol exposure can cause adverse neurobehavioral effects in children. Nevertheless, the Health Council of the Netherlands estimated in 2005 that still 35 to $50 \%$ of the Dutch pregnant women use alcohol. In this dissertation the development, evaluation and implementation of two brief interventions to reduce prenatal alcohol use are described.

Chapter 1 provides a general introduction to the topic of this dissertation. Background information is given about the consequences of alcohol use during pregnancy. The health counselling and the computer tailoring interventions which have been developed and tested in this dissertation are described. The I-Change Model is introduced as the theoretical framework of the studies and the research questions of the dissertation are presented.

Chapter 2 presents an online cross-sectional study among 158 Dutch pregnant women and their partners that aimed to investigate the influence of partners on alcohol consumption of pregnant women within the context of other psycho-social factors. This study showed that pregnant women who consumed alcohol perceived a weaker partner norm (i.e. partner's belief regarding acceptability of prenatal alcohol use) and less partner modelling (i.e. partner's alcohol abstinence during the woman's pregnancy) than pregnant women abstaining from alcohol. Moreover, the partners of pregnant women who consumed alcohol reported a weaker norm, more drinking days per week and weaker support compared to partners of pregnant women abstaining from alcohol. Perceived and reported partner norm, modelling and support were positively related, implying that the pregnant woman's perception corresponded to her partner's report of his norm, modelling and support. Multivariate analyses demonstrated that pregnant women with a higher education who perceived lower severity of harm due to prenatal alcohol use and a weaker partner norm were more likely to use alcohol. Partner norm as perceived by a pregnant woman was the most important of the perceived and reported constructs of partner influence in explaining prenatal alcohol use. 
Chapter 3 presents an online cross-sectional study among 237 Dutch partners of pregnant women. This study aimed to identify determinants of partner support to abstain from prenatal alcohol use by analysing differences between partners reporting low versus high support. This study showed that partners reporting high support were more likely to desire their spouse to abstain from alcohol use and to have received advice from their pregnant spouse or midwife that alcohol abstinence was desirable. They also had stronger perceptions that the baby would experience harm from prenatal alcohol use and that harm would be more severe. Furthermore, they saw more advantages and fewer disadvantages of providing support. They also reported more influence from their social environment encouraging their support, had greater self-efficacy and had a stronger intention to support their spouse during the remainder of the pregnancy compared to partners reporting low support.

Chapter 4 presents results from two qualitative studies. The first study among 10 midwives aimed to explore which difficulties Dutch midwives encountered when providing prenatal alcohol advice. The second study among 25 pregnant women and nine partners aimed to explore how pregnant women and partners experienced the information they receive from midwives about alcohol during pregnancy. Study 1 showed that midwives intended to advise complete abstinence, although this advice was mostly given when women indicated to consume alcohol. Midwives reported to lack good screening skills and sufficient knowledge about the mechanisms and consequences of prenatal alcohol use and were unlikely to include partners in their alcohol advice. The findings of Study 1 were confirmed in Study 2. Moreover, while partners felt ignored by midwives and pregnancy-related websites, pregnant women indicated to receive conflicting alcohol advice from their health professionals. Both pregnant women and partners considered the midwife as an important and reliable source of information on alcohol in pregnancy. This chapter concludes that midwives' alcohol advice requires improvement with regard to screening, knowledge about mechanisms and consequences of prenatal alcohol use and the involvement of the partners in alcohol advice during pregnancy. Training to Dutch midwives is recommended 
in order to increase their screening skills and their alcohol related knowledge to pregnant women. Research is needed to determine how the midwife's alcohol advice to the partner should be framed in order to optimize the partner's involvement concerning alcohol abstinence in pregnancy.

Chapter 5 presents the effect evaluation of two brief interventions to prevent prenatal alcohol use. In this cluster randomized trial, sixty Dutch midwifery practices recruited 135 health counselling (HC), 116 computer tailoring (CT) and 142 Usual Care (UC) respondents. HC respondents received counselling from their midwife according to a $\mathrm{HC}$ protocol, which consisted of seven steps, addressed in three feedback sessions. CT respondents received routine alcohol care from their midwife and three CT feedback letters via the Internet. UC respondents received routine alcohol care from their midwife. According to national guidelines, this implied that in the initial consultation, midwives advised complete alcohol abstinence to their clients who were using alcohol. After three and six months, the effect of the interventions on alcohol use was assessed. Analyses showed that CT respondents more often stopped using alcohol compared to UC respondents six months after baseline. Moreover, CT respondents with average or lower alcohol use before pregnancy or with average or lower social support more strongly reduced their alcohol use six months after baseline compared to UC respondents. Differences in alcohol use between HC respondents and UC respondents three or six months after baseline were not significant. This chapter concludes that $\mathrm{CT}$ can be effective to reduce alcohol use during pregnancy; HC was not effective. Two shortcomings may underlie the non-significant effects of the HC intervention, that is lack of statistical power (in other words, with this small number of participants the effect of HC could not be detected) and a suboptimal implementation of the intervention by midwives. Future researchers developing a $\mathrm{HC}$ intervention to reduce alcohol use during pregnancy are therefore recommended to invest more in recruitment of pregnant women and implementation by health care providers. Previous research has shown that pregnant women are reluctant to disclose their alcohol use to health professionals. Computer tailoring preserves a person's 
anonymity. This effective CT intervention is therefore recommended as an attractive intervention for pregnant women using alcohol.

Chapter 6 presents the process evaluation of the HC intervention. This qualitative study among 14 midwives aimed to explore midwives' experiences with the implementation of the $\mathrm{HC}$ intervention to prevent prenatal alcohol use. The midwives appreciated the clearly structured, comprehensive intervention, including the training, manual and intervention card. Nevertheless, contrary to the instructions, the majority of midwives had not provided alcohol counselling at the second and third consultation. The implementation was impeded by practical issues, like lack of time and the use of paper intervention cards. Due to the computerized consultations, some midwives had not filled in the paper intervention cards during the consultations as intended, but afterwards. Finally, midwives were unsure of the importance of comprehensive alcohol advice in pregnancy, which may have led to suboptimal counselling. It is recommended that future interventions take into account that midwives desire quick implementation of required activities and thus need digital intervention cards when they are used to digital reports of the consultations. Moreover, it is recommended to stress the importance of alcohol advice in midwives' training.

Finally, chapter 7 summarizes and discusses the main findings from the studies presented in chapters 2 to 6 . Methodological strengths of this dissertation are discussed, including the fact that the subject of this dissertation has been investigated from different perspectives and the assessment of the effect of the interventions in routine care. Methodological weaknesses of this dissertation include the reliance of all studies on self-reported outcome measures and the absence of an evaluation of pregnant women's experiences with the CT intervention. The discussion of the studies in this dissertation leads to five overarching, concrete recommendations to further reduce prenatal alcohol use: (1) pregnant women are recommended to use the CT intervention; (2) midwives are recommended to explore ways to improve their alcohol advice; (3) partners are recommended to strengthen their alcohol abstinence norm as a partner's alcohol abstinence norm is related to 
alcohol abstinence of his pregnant spouse; (4) in order to develop an effective HC protocol for midwives who want to help their clients to reduce their alcohol use, researchers are recommended to test the HC protocol under enhanced circumstances, which include a better training of the midwives to improve their implementation and more participants per practice to increase statistical power; and (5) because alcohol use during pregnancy is related to alcohol use before pregnancy, the Dutch national government is recommended to implement costeffective environmental universal interventions to reduce alcohol use in general. 
Samenvatting 
Steeds meer onderzoek laat zien dat ook kleine beetjes alcohol tijdens de zwangerschap kunnen leiden tot neurologische schade bij kinderen. Desondanks schatte de Gezondheidsraad in 2005 dat 35 tot 50\% van de Nederlandse zwangere vrouwen alcohol drinkt. Dit proefschrift beschrijft de ontwikkeling, evaluatie en implementatie van twee korte interventies om alcoholgebruik tijdens de zwangerschap terug te dringen.

In hoofdstuk 1 wordt een algemene introductie gegeven op het onderwerp van dit proefschrift. Er wordt achtergrondinformatie gegeven over de gevolgen van alcoholgebruik tijdens de zwangerschap. De Health Counselling en Computer Tailoring interventies die in dit proefschrift zijn ontwikkeld en getest worden beschreven. Het I-Change Model wordt geïntroduceerd als het theoretische kader van de studies en de onderzoeksvragen van het proefschrift worden gepresenteerd.

In hoofdstuk 2 wordt een online cross-sectionele studie gepresenteerd die is uitgevoerd onder 158 Nederlandse zwangere vrouwen en hun partners. Deze studie had als doel om binnen de context van verschillende psychosociale factoren te onderzoeken hoe partners het alcoholgebruik van zwangere vrouwen beïnvloeden. In deze studie rapporteerden zwangere vrouwen die alcohol dronken een zwakkere norm van hun partner (d.w.z. de overtuiging van de partner dat alcoholgebruik tijdens de zwangerschap aanvaardbaar is) en minder gedragsmodellering (d.w.z. de alcoholabstinentie van de partner tijdens de zwangerschap) dan zwangere vrouwen die geen alcohol dronken. Bovendien rapporteerden de partners van zwangere vrouwen die alcohol dronken vaker dat zij een zwakkere norm hadden, op meer dagen per week alcohol dronken en hun vrouw minder steunden bij het niet-drinken vergeleken met de partners van zwangere vrouwen die geen alcohol dronken. De norm, het gedrag en de steun van de partner zoals die werden waargenomen door de zwangere vrouw kwamen overeen met respectievelijk de norm, het gedrag en de steun zoals de partner deze zelf rapporteerde. Met multivariate analyses werd tenslotte gekeken naar de norm, het gedrag en de steun van de partner in de context van andere factoren die van invloed konden zijn op het alcoholgebruik van de zwangere vrouw. 
Deze analyses lieten zien dat zwangere vrouwen geneigd waren om alcohol te drinken als ze een hogere opleiding hadden, vonden dat de schade door prenataal alcoholgebruik niet zo ernstig was en vonden dat hun partner een zwakke norm had ten aanzien van alcoholgebruik tijdens de zwangerschap. Bij het verklaren van alcoholgebruik tijdens de zwangerschap is de norm van de partner zoals deze is waargenomen door de zwangere vrouw het belangrijkste van de waargenomen en gerapporteerde constructen van de invloed van de partner.

In hoofdstuk 3 wordt een online cross-sectionele studie gepresenteerd die is uitgevoerd onder 237 Nederlandse partners van zwangere vrouwen. Deze studie had als doel om de determinanten te identificeren van de steun van partners aan hun vrouw om geen alcohol te drinken tijdens de zwangerschap. Dit werd gedaan door de verschillen te analyseren van de partners die weinig versus veel steun verleenden aan hun zwangere vrouw. Deze studie liet zien dat de partners die rapporteerden dat ze veel steun verleenden over het algemeen vaker van hun vrouw verlangden om geen alcohol te drinken tijdens de zwangerschap dan partners die rapporteerden dat ze weinig steun verleenden. Bovendien hadden de eerst genoemde partners vaker van hun zwangere vrouw of verloskundige gehoord dat het beter was dat de vrouw geen alcohol dronk tijdens haar zwangerschap. Ze geloofden ook stelliger dat de baby beschadigd kon raken door alcohol tijdens de zwangerschap en dat deze schade ernstig was. Ze zagen meer voordelen en minder nadelen aan het verlenen van steun aan hun vrouw. Ze rapporteerden dat ze meer aanmoediging kregen van hun sociale omgeving bij het steunen van hun vrouw, ze hadden meer vertrouwen in hun eigen bekwaamheid om steun te verlenen ('zelfeffectiviteit') en een sterkere intentie om hun zwangere vrouw te steunen in de rest van de zwangerschap in vergelijking tot partners die rapporteerden dat ze weinig steun verleenden.

In hoofdstuk 4 worden de resultaten van twee kwalitatieve studies beschreven. De eerste studie, uitgevoerd onder 10 verloskundigen, had als doel om te exploreren welke moeilijkheden Nederlandse verloskundigen ervaarden bij het geven van alcoholadvies aan hun cliënten. De tweede studie, uitgevoerd onder 25 zwangere vrouwen 
en 9 partners, had als doel om te exploreren wat de ervaringen waren van Nederlandse zwangere vrouwen en partners bij het krijgen van informatie van verloskundigen over alcohol tijdens de zwangerschap. Studie 1 liet zien dat verloskundigen de intentie hadden om volledige alcoholabstinentie te adviseren, echter, dit advies werd voornamelijk gegeven aan vrouwen die daadwerkelijk aangaven dat ze alcohol dronken. Verloskundigen gaven aan dat ze goede screening vaardigheden misten en onvoldoende kennis hadden van de mechanismen en gevolgen van prenataal alcoholgebruik. Ze betrokken partners niet vaak bij hun alcoholadvies. De bevindingen van studie 1 kwamen overeen met de opvattingen van zwangere vrouwen en partners in studie 2. Daarbij voelden partners zich genegeerd door verloskundigen en zwangerschap gerelateerde websites en gaven zwangere vrouwen aan dat ze van verschillende gezondheidsprofessionals conflicterende informatie kregen. Zowel zwangere vrouwen als partners vonden dat hun verloskundige een belangrijke en betrouwbare bron was van informatie over alcoholgebruik tijdens de zwangerschap. In dit hoofdstuk wordt geconcludeerd dat het alcoholadvies van verloskundigen verbeterd moet worden zodat ze beter leren screenen, meer kennis hebben over de mechanismen en gevolgen van alcoholgebruik tijdens de zwangerschap en partners beter leren betrekken bij hun alcoholadvies. Hiervoor wordt extra training voor Nederlandse verloskundigen aanbevolen. Ook wordt aanbevolen om extra onderzoek te doen om erachter te komen hoe verloskundigen zich het beste kunnen richten tot de partner om zo een optimale betrokkenheid van de partner te krijgen bij alcoholabstinentie tijdens de zwangerschap.

In hoofdstuk 5 is de effect evaluatie beschreven van de twee korte interventies die zijn ontwikkeld om alcoholgebruik tijdens de zwangerschap te verminderen. Een cluster gerandomiseerde trial is uitgevoerd onder 135 health counselling $(\mathrm{HC})$ respondenten, 116 computer tailoring $(\mathrm{CT})$ respondenten en 142 reguliere zorg (RZ) respondenten, die geworven waren door zestig Nederlandse verloskundige praktijken. De $\mathrm{HC}$ respondenten hadden counselling gekregen van hun verloskundige volgens een HC protocol die bestond uit zeven stappen die in drie feedback sessies aan de orde kwamen. CT respondenten kregen 
regulier alcoholadvies van hun verloskundige en drie CT adviesbrieven via internet. RZ respondenten kregen regulier alcoholadvies van hun verloskundige, dat volgens de nationale richtlijnen inhield dat verloskundigen bij het intakegesprek aan hun cliënten die alcohol dronken adviseerden om hiermee te stoppen. Na drie en zes maanden werd gemeten hoeveel alcohol de respondenten dronken om zo het effect van de interventies op het alcoholgebruik vast te stellen. Analyses lieten zien dat na zes maanden CT respondenten vaker stopten met het drinken van alcohol dan RZ respondenten. Bovendien reduceerden CT respondenten die gemiddeld of minder dan gemiddeld alcohol dronken voorafgaand aan de zwangerschap en CT respondenten die gemiddeld of minder dan gemiddeld sociale steun kregen hun alcoholgebruik na zes maanden sterker dan RZ respondenten. Verschillen in alcoholgebruik tussen $\mathrm{HC}$ en RZ respondenten waren na drie of zes maanden niet significant. In dit hoofdstuk wordt geconcludeerd dat CT effectief was bij het terugdringen van alcoholgebruik tijdens de zwangerschap; de effectiviteit van HC werd niet aangetoond. Twee tekortkomingen van de studie kunnen ten grondslag liggen aan de niet-significante effecten van de HC interventie, namelijk een gebrek aan statistische power (dus, met dit beperkt aantal respondenten kon het effect van HC niet onderscheiden worden) en een suboptimale implementatie van het protocol door verloskundigen. Toekomstige onderzoekers die een HC interventie ontwikkelen om alcoholgebruik tijdens de zwangerschap terug te dringen wordt daarom aanbevolen om meer te investeren in de werving van zwangere vrouwen en in de implementatie bij gezondheidsprofessionals. Eerder onderzoek heeft laten zien dat zwangere vrouwen tegenover hun verloskundige niet altijd open zijn over hun alcoholgebruik. Computer tailoring waarborgt de anonimiteit van zwangere vrouwen. Deze effectieve $\mathrm{CT}$ interventie wordt daarom aanbevolen als een aantrekkelijke interventie voor zwangere vrouwen die alcohol gebruiken.

In hoofdstuk 6 is de procesevaluatie van de HC interventie beschreven. Deze kwalitatieve studie onder 14 verloskundigen had als doel om te exploreren wat de ervaringen van de verloskundigen waren met de implementatie van de $\mathrm{HC}$ interventie om alcoholgebruik tijdens de 
zwangerschap te verminderen. De verloskundigen waardeerden de helder gestructureerde, begrijpelijke interventie, inclusief de training, handleiding en de interventiekaart. Echter, de meerderheid van de verloskundigen had geen alcohol counselling gegeven in de tweede en derde sessie, wat in strijd was met de instructies. De onvolledige implementatie hing mogelijk samen met praktische belemmeringen, zoals gebrek aan tijd en het gebruik van papieren interventiekaarten. Omdat het consult op de computer plaatsvond, vulden de verloskundigen niet altijd de papieren interventiekaart in tijdens het consult zoals de bedoeling was, maar daarna. Tot slot twijfelden de verloskundigen over het belang van uitgebreid alcoholadvies, wat mogelijk heeft geleid tot suboptimale voorlichting. Er wordt aanbevolen dat er in toekomstige interventies rekening mee gehouden wordt dat verloskundigen de gewenste activiteiten snel moeten kunnen implementeren en dus een digitale interventiekaart nodig hebben als de rest van het consult ook op de computer plaatsvindt. Bovendien wordt aanbevolen om het belang van alcoholadvies te benadrukken in de opleiding van verloskundigen.

Tot slot zijn in hoofdstuk 7 de belangrijkste bevindingen van de studies uit hoofdstukken 2 tot en met 6 samengevat en bediscussieerd. Methodologische sterke punten van het proefschrift zijn besproken, zoals het feit dat het onderwerp van dit proefschrift vanuit verschillende perspectieven is onderzocht en dat de effectevaluatie in de reguliere zorg had plaatsgevonden. Methodologische zwakke punten van dit proefschrift waren het feit dat er uitsluitend gebruik is gemaakt van zelf-gerapporteerde uitkomstmaten en het ontbreken van een evaluatie van de ervaringen van zwangere vrouwen met de CT interventie. De discussie van de studies in dit proefschrift hebben geleid tot vijf algemene, concrete aanbevelingen om het alcoholgebruik tijdens de zwangerschap verder terug te dringen: (1) zwangere vrouwen wordt aanbevolen om de CT interventie te gebruiken; (2) verloskundigen wordt aanbevolen om te onderzoeken hoe ze hun alcoholadvies kunnen verbeteren; (3) partners wordt aanbevolen om hun norm ten aanzien van alcoholabstinentie tijdens de zwangerschap te versterken, aangezien de norm van de partner samenhangt met alcoholabstinentie van de zwangere vrouw; (4) voor de ontwikkeling van een effectief HC protocol 
voor verloskundigen die hun cliënten willen helpen bij het stoppen met drinken, worden onderzoekers aanbevolen om het $\mathrm{HC}$ protocol te testen onder verbeterde omstandigheden, dus met een betere training voor verloskundigen zodat zij de interventie goed leren te implementeren en met meer deelnemers per praktijk om de statistische power te vergroten; en (5) omdat alcoholgebruik tijdens de zwangerschap samenhangt met alcoholgebruik vóór de zwangerschap, wordt de Nederlandse overheid aanbevolen om kosteneffectieve universele interventies te implementeren waarmee alcoholgebruik onder de gehele Nederlandse bevolking wordt teruggedrongen. 
Dankwoord 
Iedereen die mij geholpen heeft bij het voltooien van dit proefschrift wil ik van harte bedanken. Een aantal van hen noem ik graag bij naam. In de eerste plaats Wim van Dalen, directeur van het Nederlands Instituut voor Alcoholbeleid STAP. Hij heeft het destijds aangedurfd om mij op dit project aan te nemen, ondanks het feit dat ik geen achtergrond had in de gezondheidsvoorlichting. Mede dankzij zijn steun en vertrouwen gedurende de cruciale eerste drie jaar is dit project geslaagd. Ik ben hem bovendien zeer dankbaar voor wat hij mij geleerd heeft over alcoholbeleid, de alcoholindustrie en beleidsbeïnvloeding; een stukje naïviteit ben ik verloren.

Mijn promotor Hein de Vries en copromotor Ciska Hoving dank ik voor de kans die zij mij hebben gegeven om onder hun supervisie te promoveren en in het bijzonder voor alle tijd die zij besteed hebben aan het project, aan het lezen en minutieus becommentariëren van de teksten. Dankzij hen heb ik dit proefschrift naar een hoger niveau weten te tillen.

Dank ook aan de leden van de leescommissie en corona voor het beoordelen van mijn proefschrift en het stellen van de vragen tijdens mijn verdediging.

Ook wil ik graag een aantal Maastrichtse collega's bedanken: Kim Eijmael voor de ontwikkeling van de computer tailoring interventie, Math Candel voor de hulp bij het analyseren van de data van hoofdstuk 5 en Leon Kolenburg bij het helpen oplossen van computer-gerelateerde problemen. Dank ook aan Eline Smit, Francine Schneider, Gera Nagelhout en andere PhD-collega van de vakgroep Health Promotion voor de grote bereidheid om te helpen, bijvoorbeeld bij het opstellen van het protocol voor de medisch-ethische commissie, het helpen met de proefpromotie en de interessante discussies tijdens de PhD-lunches.

Met veel plezier kijk ik terug op mijn tijd bij STAP. Wim, Esther, Avalon, Fieke, Joost, Sandra, Nelleke, Jurriaan, Anke, Martina, Gerrie, Jeroen, Anouk, Inge en alle andere medewerkers en stagiaires dank ik voor de inspirerende sfeer waar jullie aan hebben bijgedragen, de steun en de gezelligheid. Wat hebben we ons met z'n allen ingezet voor de 
leeftijdsgrens naar 18 jaar, voor de beperking van alcoholreclame; wat hebben we ons met z'n allen boos gemaakt als de alcoholindustrie zich weer eens richtte op jongeren en de reclamecodecommissie niet wilde ingrijpen. Als de alcoholindustrie zoete alcoholhoudende drankjes op de markt zette om meer vrouwen en kinderen aan de alcohol te krijgen. Als beleidsmakers zich lieten leiden door mooie praatjes van de alcohollobby in plaats van zich te baseren op wetenschappelijke feiten.

Een aantal experts uit het veld heeft eraan bijgedragen dat ik dit project met overtuiging en betrokkenheid heb uitgevoerd. Ik vond de inzet van Martha Krijgsheld en Diane Black van de FAS-stichting voor de preventie van het Foetaal Alcohol Syndroom in Nederland en in de rest van Europa bijzonder inspirerend. Ik heb veel respect voor hun inspanningen voor goede zorg voor en begeleiding van mensen met Foetaal Alcohol Spectrum Disorder. Ook kinderarts Rudi Kohl ben ik zeer dankbaar voor zijn welwillendheid mij te helpen. Doordat ik met hem mee mocht lopen op de FASD-polikliniek heeft hij mij laten inzien hoe belangrijk het is om schade door alcoholgebruik tijdens de zwangerschap te voorkomen. Ik was erbij toen Rudi aan een moeder moest vertellen dat haar dochtertje van anderhalf jaar de diagnose FAS kreeg. Door haar alcoholgebruik in de zwangerschap had de moeder onherstelbare schade toegebracht aan de hersenen van haar dochtertje. Zij was dus de oorzaak van de gedragsproblemen van haar dochtertje, die nooit een normaal leven zou kunnen leiden. Deze hartverscheurende ervaring op de FASDpolikliniek maakte dat ik met nog meer overtuiging wilde bijdragen aan het voorkomen van alcoholgebruik tijdens de zwangerschap.

Toen de computer-tailoring interventie effectief bleek, heb ik met gynaecoloog Kees Boer het implementatieproject uitgevoerd. Ik vond het inspirerend om met Kees samen te werken, om met hem verloskundigen en gynaecologen te overtuigen om zwangere vrouwen te verwijzen naar de interventie.

Ernst van Faassen dank ik hartelijk voor zijn hulp bij hoofdstuk 1 en zijn altijd vriendelijke bereidheid om zijn fundamentele kennis over de biochemische processen van prenatale alcoholblootstelling te delen en 
mijn vragen hierover te beantwoorden. Oom Sjoerd dank ik voor zijn algemene, wetenschappelijke schrijflessen die door het hele proefschrift van nut bleken, en voor zijn hulp bij het opstellen van de stellingen. Tot slot bedank ik buurman Albert voor zijn belangrijke bijdrage aan de totstandkoming van hoofdstuk 3 en voor de gezellige discussies over wetenschap en actuele zaken in het algemeen.

Ik heb in mijn promotieperiode heel veel geleerd tijdens de European Graduate School for Addiction Research. I am very grateful to the lecturers, especially Gerhard Buhringer, and my fellow PhD-students, especially Regina van der Meer and Lidia Segura. Thank you so much for exchanging knowledge, discussing topics on addiction and especially for having great times in Dresden, Amsterdam and Barcelona.

De bijdrage van mijn vrienden en familie was zeker zo belangrijk.

Lieve Jitske en Erica, wat heerlijk dat we nog steeds vriendinnen zijn, al sinds het begin van onze bosbouwstudie. Fantastisch dat jullie mijn paranimfen willen zijn. Ik ben verschrikkelijk blij met Annekee, Kirsten en Magda, met wie ik samen het strijkkwartet Arco ma non troppo vorm. Als het strijken te veel wordt, hebben we onze goede gesprekken. Met jullie samen spelen en praten maakt het leven tot een feestje.

Mijn moeder ben ik een speciaal dankwoord verschuldigd vanwege haar onvoorwaardelijke steun. Haar zelfdiscipline is mij tot voorbeeld geweest als ik me 's avonds, in het weekend of in vakanties naar m'n laptop sleepte om nog een uurtje te schrijven. Mijn vader heeft het voltooien van mijn proefschrift niet mee mogen maken. Het onderwerp lag hem na aan het hart; mijn promotie had ik graag met hem gevierd.

Sydo, Rosa en Nilas waren onmisbaar voor de cruciale relativering. Want als we het allemaal nuchter bekijken is commentaar op een onwetenschappelijke formulering of een afwijzing van een artikel voor een tijdschrift niet zo heel belangrijk, zeker niet wanneer je het vergelijkt met leren lezen, roze gymschoenen met gekleurde lichtjes en het tijdig krijgen van een flesje melk. Lieve Bert, de meeste dank is natuurlijk 
voor jou. Bedankt voor het overnemen van het huishouden als ik in het tuinhuisje zat te schrijven, voor je schrijftips, het meedenken, lezen en becommentariëren van de teksten, voor het mede beoordelen van het omslag. Fantastisch dat je er altijd voor me bent. 
Curriculum Vitae 
Nienke Yme (Nickie) van der Wulp was born on March 30 and moved to Heerlen in 1978. After receiving her Gymnasium diploma from the Grotius College in Heerlen in 1994, she became an exchange student for one year, lived with a host family in Roseisle Manitoba, Canada and attended Miami Collegiate, from which she received her High School diploma with honours in 1995. Nickie moved to Wageningen where she studied Forest and Nature Management. During her studies, she attended various courses in (environmental) psychology and wrote her thesis on the effects of nature on the recovery of stress. She received her master's degree in 2001. In that same year, she started working at the Social Psychology Department of the Vrije University (VU) in Amsterdam. She conducted experimental, social cognitive studies on automatic vigilance, showing that people's attention is caught faster and kept longer by negative rather than positive stimuli. After her contract ended in 2006, Nickie was applied by Alterra, research institute for our green living environment, where she conducted research on people's appreciation of their surrounding landscape.

In 2009, Nickie started working on the project described in this dissertation as an external PhD-student at the Department of Health Promotion at Maastricht University. From 2009 until the end of 2012, she conducted this project as a researcher at the Dutch Institute for Alcohol Policy STAP. From 2013 onwards, she worked on her dissertation in her spare time, which was sparse while having and raising her three children Sydo (2008), Rosa (2010) and Nilas (2014). In 2011, Nickie became a member of the management team of STAP. Also in that year, she joined the European Graduate School in Addiction Research at Dresden International University for $\mathrm{PhD} / \mathrm{MD}$ students from all over Europe. She received education from top researchers from Germany, Spain, the Netherlands and Canada (e.g. Jurgen Rehm, Wim van den Brink, Gerhard Buhringer, Marta Torrens) in research on substance use disorders. She received her diploma from Dresden International University in 2013. In that same year, Nickie became head of STAP's department Alcohol and Health, coordinating various projects on alcohol in relation to pregnancy, cancer, brains and the liver. She acquired a dissemination and implementation grant from the Netherlands Organisation for Health 
Curriculum Vitae

Research and Development (ZonMW VIMP-subsidie) and in 2014, she implemented the effective computer tailoring intervention described in this dissertation.

Since 2015, Nickie is working as general director at the Korsakov Kenniscentrum, the Dutch knowledge institute on Korsakoff's Syndrome. 
Publication List 


\section{D.D. January 17th, 2016}

\section{Published/accepted}

Van der Wulp, N.Y., Hoving, C., de Vries, H. (in press). Correlates of partner support to abstain from prenatal alcohol use: A cross-sectional survey among Dutch partners of pregnant women. Health and Social Care in the Community.

Van der Wulp, N.Y., Hoving, C., de Vries, H. (2015). Partner's influences and other correlates of prenatal alcohol use. Maternal and Child Health Journal, 19(4): 908-916.

Van der Wulp, N.Y., Hoving, C., Eijmael, K., Candel, M.J.J.M., van Dalen, W., \& de Vries, H. (2014). Reducing alcohol use during pregnancy via health counseling by midwives and internet-based computer-tailored feedback: A cluster randomized trial. Journal of Medical Internet Research, 16(12): e274.

Van der Wulp, N.Y., Hoving, C., \& de Vries, H. (2014). Dutch midwives' experiences with implementing health counselling to prevent prenatal alcohol use. Journal of Clinical Nursing, 23(21-22): 3286-3289

Van der Wulp, N.Y., Hoving, C., de Vries, H. (2013). A qualitative investigation of alcohol use advice during pregnancy: experiences of Dutch midwives, pregnant women and their partners. Midwifery, 29(11): e89-98

Lenzholzer, S., \& Van der Wulp, N.Y. (2010). Thermal experience and perception of the built environment in Dutch urban squares. Journal of Urban Design, 15(3), 375-402.

Van Dijk, T. \& Van der Wulp, N.Y. (2010). Anatomy of neighbourhood activism in defence of land conversion. Landscape and Urban Planning, 96(1), 19-28

Jostmann, N. B., Koole, S. L., Van der Wulp, N. Y., \& Fockenberg, D. A. (2005). Subliminal Affect Regulation: The Moderating Role of Action versus State Orientation. European Psychologist, 10(3), 209-217.

Van den Berg, A.E., Koole, S.L., \& Van der Wulp, N.Y. (2003). Environmental preference and restoration: (How) are they related? Journal of Environmental Psychology, 23(2),135-146 

